\title{
Lower signalizer lattices in alternating and symmetric groups
}

\author{
Michael Aschbacher \\ Communicated by Robert M. Guralnick
}

\begin{abstract}
We prove that the subgroup lattices of finite alternating and symmetric groups do not contain so-called lower signalizer lattices in the class $D \Delta$. This result is one step in a program to show that the lattices in the class $D \Delta$ are not isomorphic to an interval in the subgroup lattice of any finite group.
\end{abstract}

In 1980 in [13], Palfy and Pudlak proved that the following two statements are equivalent:

(1) Each nonempty finite lattice is isomorphic to an interval in the lattice of subgroups of a finite group.

(2) Each finite lattice is isomorphic to the lattice of congruences of some finite algebra.

Given a group $G$ and a subgroup $H$ of $G$, write $\mathcal{O}_{G}(H)$ for the lattice of overgroups of $H$ in $G$, and define a finite subgroup interval lattice to be a lattice of the form $\mathcal{O}_{G}(H)$ for some finite group $G$ and subgroup $H$ of $G$. In the thirty years since [13] appeared, the question of whether each finite lattice is a finite subgroup interval lattice has remained open and of significant interest. The general consensus seems to be that the answer is negative, and indeed that finite subgroup interval lattices constitute a small subclass of the class of all finite lattices.

Early work on the question centered on so-called M-lattices, the lattices of depth 2. Probably the high point of this effort is the reduction (in 1997 by Baddeley and Lucchini in [10]) of the existence of M-lattices as finite group interval lattices, to various questions about finite simple groups.

Each finite lattice $\Lambda$ has a greatest member $\infty$ and least member 0 . Set $\Lambda^{\prime}=$ $\Lambda-\{0, \infty\}$. Given a positive integer $m$, write $\Delta(m)$ for the lattice of subsets of an $m$-set. Given finite lattices $\Lambda_{1}$ and $\Lambda_{2}$, write $\Lambda_{1} * \Lambda_{2}$ for the lattice $\Lambda$ such that $\Lambda^{\prime}$ 
is the disjoint union of $\Lambda_{1}^{\prime}$ and $\Lambda_{2}^{\prime}$, with no member of $\Lambda_{1}^{\prime}$ comparable to a member of $\Lambda_{2}^{\prime}$. Given integers $t>1$ and $m_{1}, \ldots, m_{t}>2$, define $D \Delta\left(m_{1}, \ldots, m_{t}\right)$ to be the lattice $\Delta\left(m_{1}\right) * \cdots * \Delta\left(m_{t}\right)$.

In 2003 in [15], Shareshian made a series of conjectures related to the PalfyPudlack Question. Write $\Delta(H, G)$ for the order complex of the poset $\mathcal{O}_{G}(H)^{\prime}$. Shareshian's strongest conjecture asserts that for each finite group $G$ and subgroup $H$, the complex $\Delta(H, G)$ has the homotopy type of a wedge of spheres. His weakest conjecture asserts that $D \Delta(3,3)$ is not a finite subgroup interval lattice.

Inspired by these conjectures, Shareshian advanced the following conjecture, which sits between his other two conjectures:

Conjecture $\boldsymbol{D} \boldsymbol{\Delta}$. Given integers $t>1$ and $m_{1}, \ldots, m_{t}>2$, there does not exist a finite group $G$ and subgroup $H$ of $G$ such that the overgroup lattice $\mathcal{O}_{G}(H)$ is isomorphic to $D \Delta\left(m_{1}, \ldots, m_{t}\right)$.

In Theorem 2 of [6], the author proves:

Reduction Theorem for $\boldsymbol{D} \boldsymbol{\Delta}$. Assume $\Lambda$ is $D \Delta\left(m_{1}, \ldots, m_{t}\right)$-lattice for some integers $t>1$ and $m_{i}>2$. Assume further that $\Lambda$ is a finite subgroup interval lattice. Then there exists an almost simple group $G$ such that either

(1) $\Lambda \cong \mathcal{O}_{G}(H)$ for some $H \leq G$, or

(2) there exists a nonabelian finite simple group $L$ and $\gamma=(G, H, J) \in \mathcal{T}(L)$, such that $G=\left\langle\mathcal{W}_{0}(\gamma), H\right\rangle$ and $\Lambda \cong \Xi(\gamma)$.

Recall $G$ is almost simple if $G$ has a unique minimal normal subgroup $F^{*}(G)$, and $F^{*}(G)$ is a nonabelian simple group. Further $\mathcal{T}(L)$ is the collection of triples $\gamma=(G, H, J)$ such that $G$ is a finite group, $H \leq G$, and $J \unlhd H$ with

$$
F^{*}(H / J) \cong L
$$

Write $\mathcal{W}=\mathcal{W}(\gamma)$ for the set of signalizers for $H$ in $G$; that is those $H$-invariant subgroups $W$ of $G$ such that $H \cap W=J$. Given $G_{0} \unlhd G$, set $\hat{G}=G_{0} J$ and $\mathcal{W}_{0}=\mathcal{W}_{0}\left(\gamma, G_{0}\right)=\{W \in \mathcal{W}: W \leq \hat{G}\}$, and partially order $\mathcal{W}_{0}$ by inclusion. The poset $\Xi\left(\gamma, G_{0}\right)$ is obtained by adjoining a greatest member $\infty$ to the poset $\mathcal{W}_{0}$. It turns out (cf. 2.4) that $\Xi\left(\gamma, G_{0}\right)$ is a lattice, called a lower signalizer lattice. In the Reduction Theorem, $\mathcal{W}_{0}(\gamma)=\mathcal{W}_{0}\left(\gamma, F^{*}(G)\right)$ and $\Xi(\gamma)=\Xi\left(\gamma, F^{*}(G)\right)$.

The Reduction Theorem reduces Conjecture $D \Delta$ to two questions about sublattices of the lattice of subgroups of almost simple groups. The obvious first test cases for the two questions are the alternating and symmetric groups. 
In [8], John Shareshian and the author prove that if $G$ is alternating or symmetric, and $H \leq G$, then $\mathcal{O}_{G}(H)$ is not a $D \Delta$-lattice. This paper treats the lower signalizer lattice case by proving:

Theorem A. Let $G$ be a finite alternating or symmetric group and $H \leq G$. Let $t>1$ and $m_{1}, \ldots, m_{t}>2$ be integers. Then there does not exist a finite simple group $L$ and $J \unlhd H \leq G$ such that $\gamma=(G, H, J) \in \mathcal{T}(L), G=\left\langle W_{0}(\gamma), H\right\rangle$, and $\Xi(\gamma) \cong D \Delta\left(m_{1}, \ldots, m_{t}\right)$.

The proof of Theorem A is divided into three cases: $H$ primitive; $H$ transitive but imprimitive; $H$ intransitive. The three cases are treated in Theorem 5.23 in Section 5, Theorem 9.5 in Section 9, and Theorem 10.39 in Section 10.

Results in Sections 2 and 3 on lower signalizer lattices are also of independent interest.

See [1] for terminology and notation involving finite groups.

\section{Notation, terminology, and preliminary lemmas}

In this section we list some more specialized notation, some of which is used only in this paper. We also prove a number of preliminary lemmas.

Let $G$ be a group and $X$ and $J$ subgroups of $G$. Define

$$
\ell_{G}(J)=\left\{H \leq G: J \leq N_{G}(H)\right\},
$$

and for $X \in d_{G}(J)$, set $\mathcal{V}_{X}(J)=d_{X}(J) \cap \mathcal{O}_{X}(J \cap X)$. Define $\operatorname{ker}_{J}(G)$ to be the largest normal subgroup of $G$ contained in $J$. Write $\mathcal{M}(J)=\mathcal{M}_{G}(J)$ for the set of maximal overgroups of $J$ in $G$; that is $\mathcal{M}(J)$ is the set of maximal members of the poset $\mathcal{O}_{G}(J)-\{G\}$.

Suppose that $\Omega$ is a finite set, and set $S=\operatorname{Sym}(\Omega)$ the symmetric group on $\Omega$ and $A=\operatorname{Alt}(\Omega)$ the alternating group on $\Omega$. Let $G \leq S$.

Write $\operatorname{Fix}(G)=\operatorname{Fix}_{\Omega}(G)$ for the set of fixed points of $G$ on $\Omega$, and $\operatorname{Mov}(G)=$ $\operatorname{Mov}_{\Omega}(G)$ for the set $\Omega-\operatorname{Fix}(G)$ of points of $\Omega$ moved by $G$.

For $\Gamma \subseteq \Omega$, write $G_{\Gamma}$ for the pointwise stabilizer in $G$ of $\Gamma$; that is

$$
G_{\Gamma}=\{g \in G: \Gamma \subseteq \operatorname{Fix}(G)\} .
$$

Write $N_{G}(\Gamma)$ for the global stabilizer in $G$ of $\Gamma$, and write $G^{\Gamma}$ for the image in $\operatorname{Sym}(\Gamma)$ of $N_{G}(\Gamma)$ under the restriction map $g \mapsto g_{\mid \Gamma}$.

Let $\mathcal{P}=\mathcal{P}(\Omega)$ be the set of partitions of $\Omega$. Partially order $\mathcal{P}$ by $Q \leq P$ if $P$ is a refinement of $Q$. Thus $\mathcal{P}$ is a lattice with the obvious operators $\wedge$ and $\vee$ described in Section 3 of [5]. In particular $\mathcal{P}$ has a least member 0 and greatest member $\infty$, and, as in the introduction, $\mathcal{P}^{\prime}=\mathcal{P}-\{0, \infty\}$. Let $\mathcal{P}(G)$ be the set of $G$-invariant partitions of $\Omega$, and $\mathcal{P}^{\prime}(G)=\mathcal{P}^{\prime} \cap \mathcal{P}(G)$. 
For $Q \leq P$ in $\mathcal{P}$ and $B \in Q$, write $P_{B}$ for the partition $\{C \in P: C \subseteq B\}$ of $B$.

Given a partition $\Gamma \in \mathcal{P}$, let $N_{G}(\Gamma)$ be the subgroup of $G$ acting on $\Gamma$. Then $G_{\Gamma}$ is the kernel of the action of $N_{G}(\Gamma)$ on $\Gamma$. Write $K_{+}(\Gamma)$ for the subgroup of $A$ generated by the groups $A_{\Omega-\gamma}, \gamma \in \Gamma$. Thus $K_{+}(\Gamma)$ is isomorphic to a direct product of alternating groups.

1.1. Let $\alpha \subseteq \Omega$ and $H \leq S_{\Omega-\alpha}$ be transitive on $\alpha$. Assume $\Gamma \in \mathcal{P}(H)$ and $\gamma \in \Gamma$ with $\alpha \cap \gamma \neq \varnothing$. Then either $\alpha \subseteq \gamma$, or $\gamma \subseteq \alpha$.

Proof. Let $\omega \in \alpha \cap \gamma$. By hypothesis, $\alpha=\omega H$. Thus if $H$ acts on $\gamma$, then $\alpha \subseteq \gamma$, so we may assume $h \in H-N_{H}(\gamma)$. Then $\gamma \cup \gamma h \subseteq \operatorname{Mov}(h) \subseteq \alpha$, completing the proof.

1.2. Let $P, Q \in \mathcal{P}(\Omega)$ with $P \vee Q=\infty$. Then $S_{P} \cap S_{Q}=1$.

Proof. As $P \vee Q=\infty,|\alpha \cap \beta| \leq 1$ for all $\alpha \in P$ and $\beta \in Q$. Let $\omega \in \Omega$. Then $\omega \in \alpha \in P$ and $\omega \in \beta \in Q$, so $\alpha \cap \beta=\{\omega\}$. Then as $H:=S_{P} \cap S_{Q}$ acts on $\alpha$ and $\beta, H$ fixes $\omega$. As this holds for each $\omega \in \Omega, H=1$.

Given a lattice $\Lambda$ and $x \in \Lambda$, set $\Lambda(\leq x)=\{y \in \Lambda: y \leq x\}$.

1.3. Let $\Lambda$ be a lattice isomorphic to $\Delta(m)$ for some $m$, and $\Xi$ a sublattice of $\Lambda$ containing $\Lambda(\leq x)$ for each $x \in \Xi-\{\infty\}$. Then $\Xi=\Lambda(\leq z)$ for some $z \in \Xi$, so $\Xi \cong \Delta(d)$ for some $0 \leq d \leq m$.

Proof. Let $\mathcal{A}$ be the set of atoms of $\Lambda$, and for $x \in \Lambda$ and $\mathscr{B} \subseteq \mathcal{A}$, set

$$
\mathcal{A}(x):=\mathcal{A} \cap \Lambda(\leq x) \quad \text { and } \quad y(\mathscr{B}):=\bigvee_{b \in \mathscr{B}} b .
$$

Set $\mathcal{E}:=\mathcal{A} \cap \Xi$ and $z:=y(\mathcal{E})$. As $\Xi$ is a sublattice of $\Lambda, z \in \Xi$, so by hypothesis either $z=\infty$ or $\Lambda(\leq z) \subseteq \Xi$. In the first case as $\Lambda \cong \Delta(m), \mathcal{E}=\mathcal{A}$, and for each $x \in \Lambda, x=y(\mathcal{A}(x)) \in \Xi$, so $\Xi=\Lambda=\Lambda(\leq z)$. Thus we may assume $z \neq \infty$. Now for $x \in \Xi, \mathcal{A}(x)=\Lambda(\leq x) \cap \mathcal{A} \subseteq \mathcal{E}$, so $x=y(\mathcal{A}(x)) \leq y(\mathcal{E})=z$. Thus again $\Xi=\Lambda(\leq z)$.

1.4. Assume $n:=|\Omega| \equiv 2 \bmod 4$ and $n>2$. Then each primitive subgroup $G$ of $S$ is almost simple.

Proof. Assume $G$ is not almost simple. Then (cf. 2.5 in [4]) $G$ stabilizes an affine, diagonal, or product structure on $\Omega$.

In the first case as $n$ is even, $n=2^{a}$ for some positive integer $a$. As $n>2$, $a>1$, so $n \equiv 0 \bmod 4$, a contradiction. 
In the second case, $n=|L|^{s}$ for some nonabelian finite simple group $L$ and positive integer $s$. However $|L| \equiv 0 \bmod 4$, so $n \equiv 0 \bmod 4$, again a contradiction.

Finally in the third case, $n=m^{r}$ for some integers $m \geq 5$ and $r>1$. As $n$ is even, so is $m$, so as $r>1, n \equiv 0 \bmod 4$, once again a contradiction.

1.5. Assume $G$ is an almost simple transitive subgroup of $S$ and $n:=|\Omega|$ is a power of some prime $p$. Set $L:=F^{*}(G)$. Then either

(1) $L$ is transitive on $\Omega$, or

(2) $p=2=|G: L|$, L has two orbits $\Omega_{i}, i=1,2$, on $\Omega$, and there is an involution in $C_{A}(L)$ interchanging $\Omega_{1}$ and $\Omega_{2}$.

Proof. Since $G$ is transitive on $\Omega$, it follows that $G$ is also transitive on the set $\mathcal{O}:=\left\{\Omega_{i}: 1 \leq i \leq m\right\}$ of orbits of $L$ on $\Omega$. Set $k:=\left|\Omega_{1}\right|$. Then $k m=n=p^{e}$, so $k=p^{a}$ and $m=p^{b}$ with $a+b=e$. Further $m$ divides $|G: L|$, and hence also $|\operatorname{Out}(L)|$. As $L$ is transitive on $\Omega_{1}$ and $k=p^{a}$, it follows (cf. 3.1 in [4]) that either

(i) $p$ does not divide $|\operatorname{Out}(L)|$, or

(ii) $p=2=|\operatorname{Out}(L)|$, and either $L \cong A_{k}$, or $L \cong L_{2}(q)$ with $q$ a Mersenne prime and $k=q+1$.

In case (i), as $p^{b}=m$ divides $|\operatorname{Out}(L)|$, it follows that $m=1$, so that (1) holds. Similarly in case (ii), either (1) holds or $G=\operatorname{Aut}(L)$ and $p=m=2$, and we may assume the latter. Then for $\omega \in \Omega$ we have $L_{\omega}^{G}=L_{\omega}^{L}$, so there exists an equivalence $\alpha: \Omega_{1} \rightarrow \Omega_{2}$ of the representations of $L$ on $\Omega_{i}$. Define $t \in S$ by $\omega t=\omega \alpha$ for $\omega \in \Omega_{1}$, and $\omega t=\omega \alpha^{-1}$ for $\omega \in \Omega_{2}$. As $\alpha$ is an equivalence of $L$-representations, $t \in C_{S}(L)$. By construction, $t$ is an involution interchanging $\Omega_{1}$ and $\Omega_{2}$, so $t$ has $k$ cycles of length 2 . Then as $k$ is even, $t \in A$, so (2) holds.

1.6. Assume $G$ is a transitive subgroup of $S, n:=|\Omega|>3$, and $\Sigma=\left\{\alpha_{1}, \alpha_{2}\right\}$ is a 2-subset of $\Omega$ such that $H:=N_{G}(\Sigma)$ is transitive on $\Omega-\Sigma$. Assume $\Sigma^{G}$ is not a partition of $\Omega$.

(1) Either

(i) $G$ is 5/2-transitive on $\Omega$, that is $G$ is 2-transitive on $\Omega$, and either $G$ is 3-transitive or $G_{\Sigma}$ has two orbits on $\Omega-\Sigma$ of equal length, or

(ii) $H_{\alpha_{1}}$ has two orbits $\theta_{i}, i=1,2$, of equal length on $\Omega-\Sigma$ interchanged by $H$, and setting $\Omega_{i}:=\theta_{i} \cup\left\{\alpha_{i}\right\},\left\{\Omega_{1}, \Omega_{2}\right\}$ is a $G$-invariant partition of $\Omega$ such that $N_{G}\left(\Omega_{i}\right)$ is 2-transitive on $\Omega_{i}$ for $i=1,2$. 
(2) If $G$ is 2-transitive and affine on $\Omega$, and $G$ is not solvable, then

$$
D:=F^{*}(G) \cong E_{2^{e}},
$$

$G_{\alpha_{1}} \leq \mathrm{GL}(D), G$ is 3-transitive on $\Omega$, and either $G_{\alpha_{1}}=\mathrm{GL}(D) \cong L_{e}(2)$, or $e=4$ and $G_{\alpha_{1}} \cong A_{7}$.

(3) Assume $G$ is imprimitive and $1 \neq W \unlhd G$ with $G / W$ almost simple. Then case (ii) of (1) holds, $N_{G}\left(\Omega_{i}\right)^{\Omega_{i}}$ is affine, and $F^{*}\left(N_{G}\left(\Omega_{i}\right)^{\Omega_{i}}\right) \leq W^{\Omega_{i}}$.

Proof. We first prove (1). Set $\alpha:=\alpha_{1}$. As $\Sigma^{G}$ is not a partition of $\Omega, G_{\alpha}$ does not act on $\Sigma$ by 5.18.2 in [1]. As $H=N_{G}(\Sigma)$ is transitive on $\theta=\Omega-\Sigma$ and $|\Sigma|=2$, $H_{\alpha}=G_{\Sigma}$ and either $H_{\alpha}$ is transitive on $\theta$, or $H_{\alpha}$ has two orbits $\theta_{i}, i=1,2$, on $\theta$ of equal length $m$, interchanged by $H$. In the first case as $G_{\alpha}$ does not act on $\Sigma, G_{\alpha}$ is transitive on $\Lambda=\Omega-\{\alpha\}$, so $G$ is 3-transitive and (1i) holds. Thus we may assume the second case holds.

Next if $G_{\alpha}$ is transitive on $\Lambda$, then $G$ is $5 / 2$-transitive, and again (1i) holds, so we may assume otherwise. Thus we may assume $G_{\alpha}$ has orbits $\Delta=\Delta(\alpha)=\theta_{1}$ and $\Gamma=\left\{\alpha_{2}\right\} \cup \theta_{2}$ on $\Omega$. Therefore $G$ is a rank 3 group on $\Omega$ with parameters $k=m$ and $l=m+1$. By 16.3.2 in [1], there are nonnegative integers $\mu$ and $\lambda<k$ such that $\mu l=k(k-\lambda-1)$. Then as $(l, k)=1$, it follows that $l$ divides $k-\lambda-1$, so as $l>k>\lambda$, we conclude that $k=\lambda+1$ and $\mu=0$. Therefore by 16.4 in [1], $G$ preserves the partition $\Xi:=\left\{\omega^{\perp}: \omega \in \Omega\right\}$, where $\omega^{\perp}=\{\omega\} \cup \Delta(\omega)$. Then as $k=m$ and $l=m+1$, we get $\Xi=\left\{\Omega_{1}, \Omega_{2}\right\}$. Further as $G_{\alpha}$ is transitive on $\Omega_{2}$ and $\Omega_{1}-\{\alpha\}, N_{G}\left(\Omega_{1}\right)$ is 2-transitive on $\Omega_{1}$, so (1ii) holds, and the proof of (1) is complete.

Next assume the hypothesis of (2), and let $D=F^{*}(G)$. Then $D \cong E_{p^{e}}$ for some prime $p$, and $K=G_{\alpha} \leq \mathrm{GL}(D)$ is 3/2-transitive on $D^{\#}$. Then $G_{\Sigma}=C_{K}(d)$ for $d \in D^{\#}$ with $\alpha d=\alpha_{2}$, and $C_{K}(d)$ is $1 / 2$-transitive on $D-\{1, d\}$, so as $C_{K}(d)$ acts on $\langle d\rangle$ and $D \neq\langle d\rangle$ since $G$ is not solvable, it follows that $p=2$. Now 4.8 in [2] contains a list of subgroups of $\operatorname{GL}(D)$ transitive on $D^{\#}$ (the classification of such subgroups is due to Hering and Liebeck; see the proof of 4.8 in [2] for discussion and references). As $G_{\alpha}$ is 3/2-transitive and not solvable, we conclude from that list that (2) holds.

Finally assume the hypothesis of (3). Now $X:=N_{G}\left(\Omega_{i}\right)$ is of index 2 in $G$, so $X / U$ is almost simple, where $U:=W \cap X \unlhd G$. Next $X \leq N_{S}\left(\Omega_{1}\right)=S_{1} \times S_{2}$, where $S_{i}=S_{\Omega_{3-i}}$ acts as the symmetric group of $\Omega_{i}$. Let $\pi_{i}: X \rightarrow S_{i}$ be the $i$ th projection, and $U_{i}:=U \pi_{i}$. As $H$ is transitive on $\Xi$, we get $U_{1} \cong U_{2}$, so in particular, $U_{i} \neq 1$. As $Y_{i}:=X^{\Omega_{i}}$ is 2-transitive, $Y_{i}$ is almost simple or affine, so in particular $Y_{i}$ has a unique minimal normal subgroup $D_{i}$. Thus as $U_{i} \neq 1, D_{i} \leq U_{i}$, so if $Y_{i}$ is affine, then (3) holds. Suppose $Y_{i}$ is almost simple and set $L:=X^{\infty}$. Then $L \pi_{i}=D_{i} \leq U_{i}$ and hence $1 \neq U_{i}^{\infty}$ and $L=U^{\infty} X_{\Omega_{i}}^{\infty}$. As $X / U$ is almost sim- 
ple, it follows that $X_{\Omega_{i}}^{\infty}=D_{3-i}$ and $L=D_{1} \times D_{2}$. Then as $U_{i}^{\infty} \neq 1$ and $U \unlhd X$, it follows that $L \leq U$, and hence $X / U$ is solvable, a contradiction.

1.7. Assume $G$ is a transitive subgroup of $S, \Delta$ is a proper subset of $\Omega, N_{G}(\Delta)$ is transitive on $\Omega-\Delta$, and $1 \neq Y \unlhd N_{G}(\Delta)$ with $\Omega-\Delta \subseteq \operatorname{Fix}(Y)$. Then the following hold:

(1) If $N_{G}(\Delta)$ is 2-transitive on $\Delta$, then $Y$ is transitive on $\Delta$.

(2) If $N_{G}(\Delta)$ is 2-transitive on $\Delta$ and $\Gamma \in \mathcal{P}^{\prime}(G)$, then $\Gamma=\Delta^{G}$.

(3) If $G$ is primitive on $\Omega$, then $G$ is 2-transitive on $\Omega$ and $G$ is almost simple or affine.

(4) If $G$ is primitive and affine on $\Omega$, then $G \neq F^{*}(G) N_{G}(\Delta)$.

Proof. Let $H:=N_{G}(\Delta)$ and $\theta:=\Omega-\Delta$. We first prove (1) and (2), so we assume $H$ is 2-transitive on $\Delta$. Then $H^{\Delta}$ is primitive. As $\theta \subseteq \operatorname{Fix}(Y), Y$ is faithful on $\Delta$, so $1 \neq Y^{\Delta} \unlhd H^{\Delta}$ and hence (1) holds.

Suppose $\Gamma \in \mathcal{P}^{\prime}(G)$ and let $\gamma \in \Gamma$ with $\gamma \cap \Delta \neq 1$. By (1) and 1.1, either $\Delta \subseteq \gamma$ or $\gamma \subseteq \Delta$. In the former case $\Delta=\gamma$ as $H$ is transitive on $\theta$, and in the latter $\gamma=\Delta$ as $H$ is 2-transitive on $\Delta$. Thus (2) holds.

We next prove (3) and (4), so we assume $G$ is primitive on $\Omega$. Then statement (3) follows from 15.17 in [1]. Finally assume $G$ is affine on $\Omega$ and set $D=F^{*}(G)$. As $\theta=\operatorname{Fix}(Y)$, it follows that $C_{D}(Y)$ is regular on $\theta$, so $1 \neq C_{D}(Y)<D$ is $H$-invariant. But if $G=D H$, then as $G$ is primitive on $\Omega, H$ is irreducible on $D$, contradicting $C_{D}(Y) H$-invariant.

1.8. Let $n:=|\Omega|$.

(1) $m_{2}(S)=[n / 2]$.

(2) If $H$ is a primitive subgroup of $S$ with $m_{2}(H) \geq(n-2) / 4$, then $H$ is almost simple or affine.

Proof. Let $n_{0}:=[n / 2], E$ the subgroup of a Sylow 2-subgroup $T$ of $S$ generated by the transpositions in $T$, and $M:=N_{S}(E)$. Then $T \leq M$ so $m_{2}(M)=m_{2}(S)$. Further $m_{2}(E)=n_{0}$ and $M$ is the split extension of $E$ by $S_{n_{0}}$ with $E$ the permutation module for $M / E$. Hence by B.3.2.4 and B.2.4 in [9], $m_{2}(M)=m_{2}(E)$, establishing (1).

Assume $H$ satisfies the hypotheses of (2) but $H$ is neither affine nor almost simple. Then, replacing $H$ by a larger primitive subgroup of $S$ if necessary, and appealing to 2.2 in [4], we may assume $H$ is the stabilizer of a product structure or diagonal structure on $\Omega$. In Case I it follows from 2.2 in [4] that $H$ is a wreath product of $S_{m}$ by $S_{k}$ for some $m \geq 5$ and $k \geq 2$, so $H$ has a normal subgroup 
$D=D_{1} \times \cdots \times D_{k}$ with $D_{i} \cong S_{m}$ for $i \in I=\{1, \ldots, k\}$. In this case set $X=H$. In Case II we have $F^{*}(H)=D=D_{1} \times \cdots \times D_{k}$ with $D_{i} \cong L$ for some nonabelian simple group $L, k \geq 2$, and $H / D=X / D \times Y / D$, where $X / D=\operatorname{Sym}(I)$ and $Y / D \cong \operatorname{Out}(L)$. In each case $X$ acts on the $D_{i}$ as $\operatorname{Sym}(I)$ with kernel $D$ via $D_{i}^{x}=D_{i x}$ for $x \in X$ and $i \in I$.

Let $A$ be an elementary abelian 2-subgroup of $X$ of $\operatorname{rank} m_{2}(X)$ such that $B:=A \cap D$ is of maximal order. Claim $A=B$. Assume otherwise. Then $A$ moves some $i \in I$. Set $J=i A$, let $F$ be a complement to $A_{i}$ in $A$, and for $j \in J$ let $E_{j}$ be the projection of $B$ on $D_{j}$. As $m_{2}(A)=m_{2}(X)$, it follows that $E_{j} \neq 1$. Set $E=\left\langle E_{j}: j \in J\right\rangle$. Now $F$ is regular on $J$, so $m_{2}(F) \leq m_{2}\left(E / C_{E}(F)\right)$, and hence, as in the proof of (1), $m_{2}\left(A_{i} E\right) \geq m_{2}(A)$, contradicting the maximality of $B$. This establishes the claim.

By the claim, we have $m_{2}(X)=m_{2}(D)$, so $m_{2}(X)=k r$ where $r:=m_{2}\left(D_{i}\right)$. In Case I, $H=X$, so $m_{2}(H)=k r$ and $r=[m / 2]$ by (1). In Case II we have $H / X \cong \operatorname{Out}(L)$, so $m_{2}(H) \leq k r+3$ as $m_{2}(\operatorname{Out}(L)) \leq 3$ (cf. 2.5.12 in [11] when $L$ is of Lie type, 5.2.1 in [11] when $L$ is an alternating group, and Chapter 5 in [11] when $L$ is sporadic). Further $r=m_{2}(L)$. Thus it remains to show

$$
(n-2) / 4>k r+e
$$

where $e=0$ in Case I and $e=3$ in Case II. Assume otherwise.

In Case I, $n=m^{k}$ by 2.5 in [4], while $r=[m / 2] \leq m / 2$ by (1). Then we get $\left(m^{k}-2\right) / 4 \leq k m / 2$, so $m^{k} \leq 2(k m+1)$, a contradiction as $m \geq 5$ and $k \geq 2$.

Therefore Case II holds. Hence by 2.5 in [4], $n=m^{k-1}$ where $m:=|L|$. Observe $m=2^{r} s$ where $s \geq 15$ as $m$ is divisible by distinct odd primes. Now we find that $\left(m^{k-1}-2\right) / 4 \leq k r+3$, so

$$
\left(15 \cdot 2^{r}\right)^{k-1} \leq m^{k-1} \leq 4 k r+14
$$

again a contradiction as $k \geq 2 \leq r$.

1.9. Assume $G$ is primitive on $\Omega$ but not almost simple, and $n:=|\Omega| \geq 10$ is even. Let $\Sigma$ be a $(2, n / 2)$-partition of $\Omega, T=A_{\Sigma}$, and $z$ the fixed-point-free involution in $S$ whose cycles are the clock of $\Sigma$. Assume $z \in X:=T \cap G$ and

$$
m_{2}(X) \geq(n-2) / 4 \text {. }
$$

Then $n=16, G$ is the stabilizer in $S$ of an affine structure on $\Omega, m_{2}(X)=4$, and $G=\left\langle X^{G}\right\rangle$.

Proof. Set $Z:=\langle z\rangle$. By $1.8, G$ is affine on $\Omega$, so $F^{*}(G)=E \cong E_{2^{e}}$ for some $e \geq 4$ as $n \geq 10$ is even. Thus

$$
m_{2}(X) \geq(n-2) / 4=\left(2^{e-1}-1\right) / 2 .
$$

Set $G^{*}:=G / E$. 
As $z$ is the unique fixed-point-free involution in $X, X \cap E \leq Z$. Claim $z \in E$. Assume otherwise and let $F:=C_{E}(z)$ and $f:=m_{2}(F)$. Then $f \geq e / 2 \geq 2$. Further $[F, X] \leq E \cap X=1$. But $F$ has $2^{e} / 2^{f}=2^{e-f}$ regular orbits on $\Omega$, so each member of the set $\theta$ of orbits of $F$ on $\Sigma$ is of length at least $2^{f-1}$. Thus we have $|\theta| \leq 2^{e-1} / 2^{f-1}=2^{e-f}$, so

$$
\left(2^{e-1}-1\right) / 2 \leq m_{2}(X) \leq m_{2}\left(C_{S_{\Sigma}}(F)\right)=|\theta|=2^{e-f} .
$$

We conclude that $f \leq 2$, so as $f \geq e / 2 \geq 2$ it follows that $e=4$ and $f=2$. Thus $F=[E, z]$ so $z F \subseteq z^{E}$, and hence $Z F$ is semiregular on $\Omega$. But then each member of $\theta$ is of order 4 , so $|\theta|=2$ and $4 \leq m_{2}(X)=2$, a contradiction. This completes the proof of the claim.

By the claim, $E \cap X=Z$, so $E \leq C_{G}(Z) \leq N_{G}(X)$. Thus

$$
m_{2}\left(X^{*}\right)=m_{2}(X)-1 \geq\left(2^{e-1}-3\right) / 2 .
$$

Further $[E, X] \leq Z$, so $X^{*}$ induces a group of transvections on $E$ with center $Z$. Hence $m_{2}\left(X^{*}\right) \leq e-1$. Thus $\left(2^{e-1}-3\right) / 2 \leq e-1$, so as $e \geq 4$ we conclude that $e=4$ and $m_{2}\left(X^{*}\right)=3$.

Now as $G$ is primitive on $\Omega, G^{*}$ is irreducible on $E$. But as $m_{2}\left(X^{*}\right)=3, X^{*}$ is the radical of the parabolic of $\operatorname{GL}(E)$ stabilizing $Z$, so $G^{*}=\left\langle X^{*} G^{*}\right\rangle=\operatorname{GL}(E)$. Therefore $G^{*}=\left\langle X^{G}\right\rangle^{*}$, so $Y=\left\langle X^{G}\right\rangle$ is irreducible on $E$. Hence we obtain that $E=\left\langle E^{Y}\right\rangle \leq Y$, so $G=E Y=Y$, completing the proof.

\section{Lower signalizer lattices}

In this section we assume:

Hypothesis 2.1. $G$ is a finite group, $L$ is a nonabelian finite simple group, $\gamma=$ $(G, H, J) \in \mathcal{T}(L)$, and $G_{0}$ is a normal subgroup of $G$.

Notation 2.2. Set $\hat{G}:=G_{0} J$, write $H_{0}$ for the preimage in $H$ of $F^{*}(H / J)$, and let

$$
\mathcal{W}_{0}:=\left\{W \in \mathcal{W}\left(\gamma, G_{0}\right): W \leq \hat{G}\right\} .
$$

Partially order $\mathcal{W}_{0}$ by inclusion, and let $\Xi=\Xi\left(\gamma, G_{0}\right)$ be the poset obtained by adjoining a greatest member $\infty$ to the poset $\mathcal{W}_{0}$. Observe $J$ is the least element of $\Xi$; we sometimes write 0 for $J$ regarded as a member of $\Xi$. Set $W_{0}^{\prime}:=W_{0}-\{0\}$, and for $W \in W_{0}^{\prime}$ let

$$
\mathcal{W}_{0}(>W):=\left\{U \in \mathcal{W}_{0}: W<U\right\} \quad \text { and } \quad \mathcal{W}_{0}(<W):=\left\{U \in \mathcal{W}_{0}^{\prime}: U<W\right\} \text {. }
$$

Define $\mathcal{W}_{0}(\geq W)$ and $\mathcal{W}_{0}(\leq W)$ similarly. For $X \in \mathcal{d}_{G}(J)$, set $\bar{X}:=X J$. 
Remark 2.3. We call a lattice of the form $\Xi\left(\tau, X_{0}\right)$, for some $\tau=\left(X, H_{X}, J_{X}\right) \in$ $\mathcal{T}(L)$ and $X_{0} \unlhd X$, a lower signalizer lattice. From (the proof of) 2.11.2 in [6], $\Xi(\tau)$ is isomorphic to the dual of the poset $\Delta\left(\tau, X_{0}\right)$ defined in Section 2 of [6]. Thus we can appeal to results from [6] on such posets.

2.4. (1) $\Xi:=\Xi\left(\gamma, G_{0}\right)$ is a lattice.

(2) Suppose $\infty \neq W_{i} \in \Xi$ for $i=1,2$. Then

$$
W_{1} \wedge W_{2}=W_{1} \cap W_{2},
$$

and if $W_{1} \vee W_{2} \neq \infty$, then $W_{1} \vee W_{2}=\left\langle W_{1}, W_{2}\right\rangle$.

Proof. The lemma follows from (the proof of) parts (2) and (3) of 2.11 in [6], which use the isomorphism of Remark 2.3.

2.5. Let $H \leq M \leq G$. Then

(1) $\tau_{M}:=(M, H, J) \in \mathcal{T}(L)$.

(2) $\Xi_{M}:=\Xi\left(\tau_{M}, G_{0} \cap M\right)$ is a lower signalizer lattice, and a sublattice of $\Xi$.

(3) For $W \in \Xi_{M}, \mathfrak{W}_{0}(\leq W) \subseteq \Xi_{M}$.

(4) The inclusion map is an isomorphism of

$$
\Xi\left(\tau_{\left\langle W_{0}\left(\gamma, G_{0}\right), H\right\rangle},\left\langle\mathcal{W}_{0}\left(\gamma, G_{0}\right), H\right\rangle \cap G_{0}\right)
$$

with $\Xi$.

Proof. The proof is straightforward.

2.6. $J=1$ if and only if $H$ is almost simple.

Proof. As $\gamma \in \mathcal{T}(L), H / J$ is almost simple. Thus if $J=1$, then $H$ is almost simple. Conversely if $H$ is almost simple, then $F^{*}(H)$ is the unique minimal normal subgroup of $H$, so if $J \neq 1$, then $F^{*}(H) \leq J$. This is impossible as $H / F^{*}(H)$ is solvable, whereas $H / J$ is not.

2.7. Assume $V \in \mathcal{W}_{0}$. Then:

(1) For each $W \in \mathcal{V}_{V}(H), W \in \mathcal{W}_{0}$.

(2) If $F^{*}(H V) \leq V$, then $F^{*}(H V) J \in W_{0}$.

Proof. Part (1) follows from 2.5.1 in [6]. Assume $F^{*}(H V) \leq V$. Then we obtain $J \leq F^{*}(H V) J \in d_{V}(H)$, so (1) implies (2).

2.8. Assume $X_{i} \in \mathcal{d}_{\hat{G}}(H), 1 \leq i \leq 3$, with $X_{1} \unlhd X_{2} \leq X_{3}, H \cap X_{3} \leq X_{2}, \bar{X}_{1} \in \mathcal{W}$, and $X_{2} / X_{1}$ solvable. Then $\bar{X}_{i} \in \mathcal{W}_{0}$ for $1 \leq i \leq 3$. 
Proof. If $\bar{X}_{2} \in \mathcal{W}$, then as $H \cap X_{3} \leq X_{2}$, we have $H \cap X_{3}=H \cap X_{2} \leq J$, so $\bar{X}_{3} \in \mathcal{W}$. Then as $X_{3} \leq \hat{G}$, the lemma holds. So assume $\bar{X}_{2} \notin \mathcal{W}$. Applying 3.6.2 in [6] to $\bar{X}_{1}, X_{2}$ in the role of $V, W$, we conclude that $P:=N_{X_{2}}\left(\bar{X}_{1}\right)$ is $H$-invariant with $\bar{X}_{1} \cap X_{2} \leq P$ and $L \cong P /\left(\bar{X}_{1} \cap X_{2}\right)$. But $\bar{X}_{1} \cap X_{2}=X_{1}\left(J \cap X_{2}\right)$, and $P / X_{1}$ is a subgroup of the solvable group $X_{2} / X_{1}$, so the image

$$
L \cong P /\left(X_{1}\left(J \cap X_{2}\right)\right)
$$

of $P / X_{1}$ is solvable, a contradiction.

2.9. Assume $V \in \mathcal{W}_{0}$ and $M \in \mathcal{O}_{G}(H V)^{\prime}$ with

- $F^{*}(H V) \leq F^{*}(M) \leq \hat{G}$,

- $F^{*}(H V) \leq V$, and

- $N_{F^{*}(M)}\left(F^{*}(H V)\right) / F^{*}(H V)$ is solvable.

Then $F^{*}(M) J$ and $N_{F^{*}(M)}\left(F^{*}(H V)\right) J$ are in $\mathcal{W}_{0}$.

Proof. Let $X_{1}=F^{*}(H V), X_{3}=F^{*}(M)$, and $X_{2}=N_{X_{3}}\left(X_{1}\right)$. By 2.7.2, we have $\bar{X}_{1} \in \mathcal{W}_{0}$, and by hypothesis $X_{2} / X_{1}$ is solvable. As $H$ acts on $X_{1}$, it follows that $H \cap X_{3} \leq N_{X_{3}}\left(X_{1}\right)=X_{2}$. Thus the lemma follows from 2.8.

2.10. Assume for all $V \in \mathcal{W}_{0}$ that:

(a) $F^{*}(H V) \leq V$.

(b) There exists $M(V) \in \mathcal{M}(H V)$ such that

$$
F^{*}(H V) \leq F^{*}(M(V)) \leq \hat{G}
$$

and $N_{F^{*}(M(V))}\left(F^{*}(H V)\right) / F^{*}(H V)$ is solvable.

Then the following hold:

(1) $F^{*}(H V) J$ and $F^{*}(M(V)) J$ are in $W_{0}$.

(2) Let $\mathcal{X}$ consist of the subgroups $X$ of $F^{*}(H)$ such that $X \unlhd H$ and

$$
C_{F^{*}(H)}(X) \leq X \text {. }
$$

Assume in addition that:

(c) For each $X \in X, N_{G}(X) \leq M(J)$, and for all $V \in W_{0}$,

$$
F^{*}(M(J)) \leq F^{*}(M(V)) .
$$

Then either $\Xi$ is connected or $F^{*}(M(J)) \leq J$. 
(3) Assume the hypothesis of (2) and assume $\Xi$ is disconnected and for each $V \in \mathcal{W}_{0}, K(V)$ is a normal subgroup of $M(V)$ containing $F^{*}(M(V))$ such that $K(V) / F^{*}(M(V))$ is solvable, $K(V) \leq \hat{G}$, and $K(J) \leq K(V)$. Then we have $K(V) J \in \mathcal{W}_{0}$ and $K(J) \leq J$.

Proof. Part (1) follows from 2.7.2 and 2.9, so assume the hypothesis of (2). Let $P:=F^{*}(M(J))$, let $V$ be a minimal member of $W_{0}^{\prime}$, and set $Q:=F^{*}(M(V))$ and $R:=F^{*}(H V)$. We may assume $P \not \leq J$, and it remains to show $\Xi$ is connected. For $U \in W_{0}^{\prime}$, let $\mathcal{C}(U)$ be the connected component of $\Xi^{\prime}$ containing $U$. As $P \not \leq J, \bar{P} \in W_{0}^{\prime}$ by (1). By (c), $P \leq Q$, and $\bar{Q} \in \mathcal{W}_{0}$ by (1), so $\bar{Q} \in \mathcal{W}_{0}^{\prime}$ and $\mathcal{C}(\bar{P})=\mathcal{C}(\bar{Q})$. Similarly $\bar{R} \in W_{0}$ by (1), and $\bar{Q} \geq \bar{R} \leq V$ by (a) and (b), so if $R \not J$, then $\bar{R} \in W_{0}^{\prime}$ and $\mathscr{C}(V)=\ell(\bar{Q})=\ell(\bar{P})$. Thus $\Xi$ is connected unless we can choose $V$ with $\mathscr{C}(V) \neq \mathcal{C}(\bar{P})$ and $R \leq J$, and we may assume the latter.

As $R \leq J, R \in \mathcal{X}$. Also $R \leq S=F^{*}(H)=F^{*}(H J) \leq F^{*}(M(J))=P$ by (b), and $H V \leq N_{G}(R) \leq M(J)$ by (c). Then $V$ acts on $P$ so $\langle V, \bar{P}\rangle=V P$. Hence as $\mathcal{C}(V) \neq \mathcal{C}(\bar{P}), V P \notin \mathcal{W}_{0}$ and $V \cap \bar{P}=J$ by 3.8 in [6]. Next by 3.9.3.c in [6], $U:=N V(\bar{P}) \in \mathcal{W}_{0}^{\prime}$, so $U=V$ by minimality of $V$. Then by 3.9.3.d in [6], $\theta(V) \leq N_{\bar{P}}(V)$, where $\theta(V)$ is defined in 3.2 of [6]. Therefore as $V \cap \bar{P}=J$, $\theta(V) \leq N_{\bar{P}}(J)$. As $S:=F^{*}(H) \leq J$ by (a), $S=F^{*}(J)$, so $\theta(V) \leq N_{\bar{P}}(S)$. But $S \leq J \cap P$, so $N_{\bar{P}}(S) / J=N_{P}(S) J / J \cong N_{P}(S) /(J \cap P)$ is an image of $N_{P}(S) / S$, which is solvable by (b), whereas $\theta(V) / J \cong L$ by 3.2.4 in [6], a contradiction. This completes the proof of (2).

Assume the hypothesis of (3). By (1), $\bar{Q} \in \mathcal{W}_{0}$, so as $Q^{\prime}=K(V) \unlhd M(V)$ and $Q^{\prime} / Q$ is solvable, $\bar{Q}^{\prime} \in \mathcal{W}_{0}$ by 2.8 applied with $X_{1}=Q$ and $X_{2}=X_{3}=Q^{\prime}$. Assume $P^{\prime}=K(J) \not \leq J$. As $\Xi$ is disconnected, we can choose $V$ with $\mathscr{C}(V) \neq$ $\digamma\left(\bar{P}^{\prime}\right)$. By hypothesis, $\bar{P}^{\prime} \leq \bar{Q}^{\prime}$, so $\mathcal{C}\left(\bar{P}^{\prime}\right)=\mathcal{C}\left(\bar{Q}^{\prime}\right)$. Further $\bar{Q} \leq \bar{Q}^{\prime}$ and from the proof of (2), $\bar{Q} \geq \bar{R} \leq \bar{V}$, so if $R \not J$, then

$$
\ell(V)=\ell(\bar{Q})=\ell\left(\bar{Q}^{\prime}\right)=\ell\left(\bar{P}^{\prime}\right),
$$

contrary to the choice of $V$. Thus $R \leq J$, and then the argument in the last paragraph of the proof of (2) applied to $P^{\prime}$ in place of $P$ supplies a contradiction.

2.11. Let $\mathscr{H}$ be the set of $H$-invariant subgroups $X$ of $H_{0}$ such that $H_{0}=X J$. Then $\mathscr{H}$ has a least element $H_{*}$.

Proof. Suppose $H_{i} \in \mathscr{H}$ for $i=1,2$ and let $J_{i}:=J \cap H_{i}, H_{3}:=\left[H_{1}, H_{2}\right]$, and $H^{*}:=H / J_{1}$. As $H_{i} \unlhd H$ for $i=1,2$, we get $H_{3} \leq H_{1} \cap H_{2}$, so it suffices to show that $H_{3} \in \mathscr{H}$.

Now $H_{1}^{*} \cong H_{1} / J_{1} \cong H_{1} J / J=H_{0} / J \cong L$ is nonabelian simple and normal in $H^{*}$. As $H_{0}=H_{1} J$, it follows that $H_{0}^{*}=H_{1}^{*} J^{*}$ and $H_{1}^{*} \cap J^{*}=J_{1}^{*}=1$, so 
$H_{0}^{*}=H_{1}^{*} \times J^{*}$. As $H_{0}^{*}=H_{2}^{*} J^{*}$ and $H_{1}^{*}$ is nonabelian simple, we get

$$
H_{1}^{*}=\left[H_{1}^{*}, H_{2}^{*}\right]=\left[H_{1}, H_{2}\right]^{*}=H_{3}^{*},
$$

so $H_{1}=H_{3} J_{1}$. Thus $H_{0}=H_{1} J=H_{3} J$, so $H_{3} \in \mathscr{H}$, completing the proof.

2.12. Let $X \in \mathcal{d}_{\hat{G}}(H)$. Then either $\bar{X} \in \mathcal{W}_{0}$ or $H_{*} \leq X$.

Proof. If $\bar{X} \in \mathcal{W}$, then as $\bar{X} \leq \hat{G}$, we have $\bar{X} \in \mathcal{W}_{0}$. So assume $\bar{X} \notin \mathcal{W}$. Then by 3.5 in [6], $H_{0}=\left(X \cap H_{0}\right) J$. Thus $X \cap H_{0}$ is in the set $\mathscr{H}$ of 2.11 , so $H_{*} \leq X$ by 2.11 .

2.13. Let $W \in \mathcal{W}_{0}, X \in \mathcal{d}_{\hat{G}}(H W)$, and $Y \leq X \cap W$ with $Y \unlhd X$ and $X / Y$ solvable. Then $W X \in \mathcal{W}_{0}$.

Proof. Assume $W X \notin \mathcal{W}_{0}$. As $X \leq \hat{G}$, also $W X \leq \hat{G}$, so if $W X \in \mathcal{W}$, then also $W X \in \mathcal{W}_{0}$. Thus we have $W X \notin \mathcal{W}$. Then by 3.6.2 in [6], $V:=N_{X}(W)$ satisfies $Z=W \cap X \leq V$ and $L \cong V / Z$. Let $X^{*}=X / Y$, so that $X^{*}$ is solvable. As $Y \leq Z \leq V$, it follows that $L \cong V / Z \cong V^{*} / Z^{*}$ is a section of the solvable group $X^{*}$, a contradiction.

2.14. Assume $W \in W_{0}$ and $W H$ is almost simple. Then $W=1$.

Proof. If not, then as $W \unlhd W H, F^{*}(W H) \leq W$. Thus $W H / W$ is solvable, contradicting $W H / W$ almost simple.

2.15. Assume $U \in W_{0}^{\prime}$ and $Y:=U H$ is represented as a 2-transitive group of permutations on a set $\Gamma$. Set $K:=Y_{\Gamma}$ and let $D$ be the preimage in $Y$ of $F^{*}\left(Y^{\Gamma}\right)$. Assume either $K<U$ or $K \leq J$. Then

(1) $Y^{\Gamma}$ is affine.

(2) $D^{\Gamma} \leq U^{\Gamma}$.

(3) $D \leq U$ and $\bar{D} \in W_{0}$.

Proof. As $Y^{\Gamma}$ is 2-transitive, $Y^{\Gamma}$ is almost simple or affine. In particular $F^{*}\left(Y^{\Gamma}\right)$ is the unique minimal normal subgroup of $Y^{\Gamma}$. By hypothesis either $K<U$ or $K \leq J$. In the latter case as $U \in \mathcal{W}_{0}^{\prime}$, again $K<U$. Thus $1 \neq U^{\Gamma} \unlhd Y^{\Gamma}$, so (2) holds.

Next if $Y^{\Gamma}$ is almost simple, then $Y^{\Gamma} / U^{\Gamma}$ is solvable by (2), so as $K \leq U$, also $U H / U$ is solvable, a contradiction. Therefore (1) holds.

As $K \leq U$, (2) says that $D \leq U$. Thus $\bar{D} \in \mathcal{W}_{0}$ by 2.7.1, so (3) holds. 
2.16. Let $V \in \mathcal{W}_{0}$ and $\operatorname{set} \alpha:=(G, H V, V)$. Then

(1) $\alpha \in \mathcal{T}(L)$ and $G, \alpha, G_{0}$ satisfies Hypothesis 2.1.

(2) $\mathcal{W}_{0}(\alpha)=\left\{W \in \mathcal{W}_{0}: V \leq W\right\}$.

Proof. Straightforward.

\section{$3 D \Delta\left(m_{1}, \ldots, m_{t}\right)$-lower signalizer lattices}

In this section we assume:

Hypothesis 3.1. $G$ is an almost simple finite group, $L$ is a nonabelian finite simple group, $\gamma:=(G, H, J) \in \mathcal{T}(L), \Lambda:=\Xi(\gamma)$ is a $D \Delta\left(m_{1}, \ldots, m_{t}\right)$-lattice for some integers $t>1, m_{i}>2$, and $G=\left\langle\mathcal{W}_{0}, H\right\rangle$. For $X \in \mathcal{l}_{G}(J)$, set $\bar{X}:=X J$.

Remark 3.2. Observe that Hypothesis 2.1 is satisfied with $G_{0}=F^{*}(G)$. We adopt Notation 2.2. In addition let $W_{0}^{*}$ be the set of minimal members of the poset $W_{0}^{\prime}$, and let $\mathcal{W}_{0}^{!}$be the set of maximal members of $\mathcal{W}_{0}^{\prime}$. Define $H_{*}$ as in 2.11 .

3.3. $G=\left\langle\mathcal{W}_{0}^{*}, H\right\rangle$.

Proof. As $\Lambda$ is a $D \Delta\left(m_{1}, \ldots, m_{t}\right)$-lattice, for each $W \in \mathcal{W}_{0}$,

$$
W=\left\langle U: U \in \mathcal{W}_{0}^{*} \text { and } U \leq W\right\rangle
$$

(cf. 3.4). Hence the lemma follows as $G=\left\langle\mathcal{W}_{0}, H\right\rangle$ by 3.1.

3.4. Let $\mathcal{C}$ be a connected component of $\Lambda^{\prime}, \Sigma:=\ell \cup\{0, \infty\}$, and $\mathcal{C}^{*}:=\ell_{\cap} \mathfrak{W}_{0}^{*}$. For $\alpha \subseteq \ell^{*}$, set $L_{\alpha}:=\langle W: W \in \alpha\rangle$, with $L_{\ell^{*}}:=\infty$ and $L_{\varnothing}:=J$. Let $\mathfrak{C}$ be the lattice of all subsets of $\mathcal{C}^{*}$. Then the following hold:

(1) $\Sigma \cong \Delta\left(m_{i}\right)$ for some $1 \leq i \leq t$.

(2) The map $\alpha \mapsto L_{\alpha}$ is an isomorphism of posets of $\mathfrak{C}$ with $\Sigma$.

(3) $L_{\alpha \cup \beta}=\left\langle L_{\alpha}, L_{\beta}\right\rangle$ and $L_{\alpha \cap \beta}=L_{\alpha} \cap L_{\beta}$.

(4) $\left\langle e^{*}\right\rangle \notin W_{0}$.

Proof. Part (1) follows as $\Lambda$ is a $D \Delta\left(m_{1}, \ldots, m_{t}\right)$-lattice. Then (1) and 2.4 imply (2), (2) implies (3), and (1) and (2) imply (4), since $\left\langle\mathcal{C}^{*}\right\rangle=L \iota^{*}=\infty$.

3.5. The following hold:

(1) Assume $U, V \in W_{0}$ with $V<U$. Then $V=N_{U}(H V)$.

(2) $|U: V| \neq 2$.

(3) $|U: J| \neq 2$. 
Proof. If (2) fails, then $[H, U] \leq V \leq H V$, so (1) supplies a contradiction. Also (2) implies (3). Thus it suffices to prove (1).

Assume the setup of (1), with $V<N_{U}(H V)$. By 2.5.1 in [6], $N_{U}(H V) \in \mathfrak{W}_{0}$, so we may take $U=N_{U}(H V)$. Form $\alpha:=(G, H V, V)$ as in 2.16. By that lemma, $\Gamma_{1}:=W_{0}(\geq V) \cup\{\infty\}$ is a sublattice of $\Lambda$. Let $\Gamma_{0}$ be the connected component of $\Gamma_{1}^{\prime}$ containing $U$, and $\Gamma:=\Gamma_{0} \cup\{0, \infty\}$. From 3.4, $\Gamma \cong \Delta(m)$ for some $m>1$. Now $U$ acts on $H V$ and $V=H V \cap U$, so $U$ is represented as a group of automorphisms of $\Gamma$ via conjugation. Further $U \in \Gamma$. Let $\Gamma^{*}$ be the minimal members of $\Gamma, \mu:=\left\{W \in \Gamma^{*}: W \leq U\right\}, \eta:=\Gamma^{*}-\mu$, and $X:=\langle\eta\rangle$. Then we find that $X \cap U=V$ and $U$ acts on $\mu$ and $\Gamma^{*}$, and hence also on $\eta$ and $X$. By 3.4.4, $U X \notin W_{0}$, so by 3.9.3 in [6], setting $U_{1}:=N_{U}(X)$ and $X_{1}:=N_{X}(U)$, we obtain that $V=U_{1} \cap X_{1} \unlhd U_{1} X_{1}=Y$, and setting $Y^{*}=Y / V, Y^{*}=X_{1}^{*} \times U_{1}^{*}$ with $H_{*}^{*} \cong L$ a full diagonal subgroup of $Y^{*}$. As $H$ centralizes $U^{*}$, this is a contradiction.

\subsection{The following hold:}

(1) For $W \in W_{0}, F^{*}(G) \not \leq W H$, so $\mathcal{M}(W H) \neq \varnothing$.

(2) $|\mathcal{M}(H)|>1$.

Proof. Suppose (1) fails for some $W$. Then $W=1$ by 2.14 , so $F^{*}(G) \leq H$. Thus for each $U \in \mathcal{W}_{0}, F^{*}(G) \leq U H$, so $U=1$, contrary to $\Lambda^{\prime} \neq \varnothing$. This establishes statement (1). Then (1) and 3.3 imply (2).

3.7. Let $\mathcal{C}$ be a connected component of $\Lambda^{\prime}$, and adopt the notation of 3.4. Assume $Y \unlhd X \leq G$ with $H \leq X$, and set $\mathscr{D}:=\left\{d \in \mathcal{C}^{*}: L_{d} \leq X\right\}$. For $\delta \subseteq \mathscr{D}$, set $Y_{\delta}:=L_{\delta} \cap Y$. Set $\mathscr{B}:=\left\{b \in \mathscr{D}: Y_{b} \leq J\right\}$ and $\mathcal{A}:=\mathscr{D}-\mathscr{B}$. Then for each $\alpha \subseteq \mathcal{A}$ and $\beta \subseteq \mathscr{B}$ :

(1) $L_{\alpha}=\bar{Y}_{\alpha}$.

(2) $Y_{\alpha}=\left\langle Y_{a}: a \in \alpha\right\rangle$.

(3) The map $\alpha \mapsto Y_{\alpha}$ is an isomorphism of the lattice $\mathfrak{A}$ of all subsets of $\mathcal{A}$ with $\mathcal{V}_{Y_{\mathcal{A}}}(H)$.

(4) $L_{\beta}$ acts on $Y_{\alpha}$.

(5) $Y_{\beta} \subseteq J$.

Proof. Let $a \in \alpha, b \in \beta$. By 2.7, $\bar{Y}_{a} \in \mathcal{W}_{0}$ and as $a \in \mathcal{A}, Y_{a} \not \leq J$, so $\bar{Y}_{a} \in W_{0}^{\prime}$. Then as $\bar{Y}_{a} \leq L_{a} \in W_{0}^{*}, L_{a}=\bar{Y}_{a}$. By 3.4,

$$
L_{\alpha}=\left\langle L_{a}: a \in \alpha\right\rangle=\left\langle\bar{Y}_{a}: a \in \alpha\right\rangle=Z_{\alpha} J,
$$


where $Z_{\alpha}:=\left\langle Y_{a}: a \in \alpha\right\rangle$. Thus $Z_{\alpha} \leq L_{\alpha} \cap Y=Y_{\alpha}$ and hence (1) holds. Further $Y_{\alpha}=L_{\alpha} \cap Y=Z_{\alpha} J \cap Y=Z_{\alpha}(Y \cap J)=Z_{\alpha}$ as $J \cap Y \leq Y_{a} \leq Z_{\alpha}$. Thus (2) holds. Then (1) and (2) imply the map $\varphi: \mathfrak{A} \rightarrow \mathcal{V}_{Y_{\mathcal{Q}}}(H)$ of (3) is an injective map of lattices. By 2.7, $\varphi$ is a surjection, so (3) holds.

Next $Y_{a, b}=L_{a, b} \cap Y$ is $H$-invariant, so $\bar{Y}_{a, b} \in \mathcal{W}_{0}$ by 2.7. Further we have $Y_{a} \leq Y_{a, b} \leq L_{a, b}$, so $L_{a}=\bar{Y}_{a} \leq \bar{Y}_{a, b} \leq L_{a, b}$. Therefore by 3.4.2, $\bar{Y}_{a, b}=L_{a}$ or $L_{a, b}$. But in the latter case, $L_{b}=L_{b} \cap L_{a, b}=L_{b} \cap Y_{a, b} J \leq\left(L_{b} \cap Y\right) J=J$, a contradiction. Thus $\bar{Y}_{a, b}=L_{a}$, so $Y_{a, b}=Y_{a}$. Thus $Y_{a}$ is $L_{b}$-invariant, so by 3.4.2, $L_{\underline{\beta}}=\left\langle L_{b}: b \in \beta\right\rangle$ acts on $Y_{\alpha}=\left\langle Y_{a}: a \in \alpha\right\rangle$, establishing statement (4). Finally $\bar{Y}_{\beta} \leq L_{\beta}$, so $\bar{Y}_{\beta}=L_{\delta}$ for some $\delta \subseteq \beta$. But if $b \in \delta$, then

$$
L_{b}=L_{b} \cap L_{\delta} \leq L_{b} \cap Y J=\left(L_{b} \cap Y\right) J=J,
$$

a contradiction. Thus $L_{\beta} \cap Y \leq J$, establishing (5).

3.8. Assume $D \in \ell_{G}(H)$ with $\bar{D} \in \mathcal{W}_{0}^{\prime}$. Let $\mathcal{C}$ be the connected component of $\bar{D}$ in $\Lambda^{\prime}$, and adopt the notation of 3.4. Then $\bar{D}=L_{\alpha}$ for some $\varnothing \neq \alpha \subset \mathcal{C}^{*}$, and setting $\beta:=\ell^{*}-\alpha, W=L_{\beta}$ does not act on $D$.

Proof. Assume otherwise, and let $X:=W D H$ and $X^{*}:=X / D$. By 3.4.4, we have $W D \notin \mathcal{W}_{0}$, so $H_{*} \leq W D$ by 2.12 . Let $V$ be the preimage in $W$ of $H_{*}^{*}$. As $H_{*}$ and $W$ are $H$-invariant, $V$ is $H$-invariant. Thus we get $\bar{V} \in \mathcal{W}_{0}$ by 2.7.1. Now $H_{*} \leq V D$ so $\bar{V} \bar{D} \notin W_{0}$. Notice $\bar{V} \cap \bar{D} \leq W \cap \bar{D}=L_{\beta} \cap L_{\alpha}=J$ by 3.4.3. Set $V_{1}:=N_{\bar{V}}(\bar{D})$ and $D_{1}:=N_{\bar{D}}(\bar{V})$; then $J=V_{1} \cap D_{1}$ and $V_{1}, D_{1} \in \mathcal{W}_{0}$. Set $X_{1}:=V_{1} D_{1}$ and $X_{1}^{*}:=X / J$. By 3.9.3 in [6], $V_{1}^{*} \cong D_{1}^{*} \cong L$ and $H_{*}^{*}$ is a full diagonal subgroup of $X_{1}^{*}$. Thus $V_{1}, D_{1} \in W_{0}^{*}$, so $V_{1}=L_{b}$ and $D_{1}=L_{a}$ for some $a \in \alpha$ and $b \in \beta$. Thus as $|\mathcal{C}| \geq 3$, it follows that $V_{1} D_{1}=L_{a, b} \in \mathcal{C}$, contradicting $H_{*} \leq V_{1} D_{1}$.

\section{Semisimple subgroups}

In this section we assume:

Hypothesis 4.1. Hypothesis 3.1 holds, $M \in \mathcal{O}_{G}(H)$, and $D \unlhd M$ is the direct product of a set $\mathscr{L}=\left\{D_{1}, \ldots, D_{m}\right\}$ of subgroups permuted transitively by $H$, with $m>1$ and $H_{*} \leq D$.

Notation 4.2. For $X \leq M$ and $E \in \mathscr{L}$, set $X_{E}:=X \cap E, X_{D}:=X \cap D$, and write $\tilde{X}_{E}$ for the projection of $X_{D}$ on $E$. Set

$$
\tilde{X}:=\prod_{E \in \mathscr{L}} \tilde{X}_{E}
$$


4.3. The following hold:

(1) $\tau_{M}:=(M, H, J) \in \mathcal{T}(L)$.

(2) $\Xi_{M}:=\left(\tau_{M}, F^{*}(G) \cap M\right)$ is a lower signalizer lattice, and a sublattice of $\Lambda$.

(3) For $\infty \neq W \in \Xi_{M}$, $W_{0}(\leq W) \subseteq \Xi_{M}$.

Proof. This is immediate from 2.5.

4.4. Let $V \in \hat{\Xi}_{M}:=\Xi_{M}-\{\infty\}$. Then:

(1) For each $E \in \mathscr{L}, \tilde{H}_{* E} \tilde{V}_{E} / \tilde{V}_{E} \cong L$.

(2) $\tilde{V} J \in W_{0}$.

(3) $H_{* D} \tilde{V} / \tilde{V}$ is a full diagonal subgroup of $\tilde{H}_{* D} \tilde{V} / \tilde{V}$.

Proof. The proofs of 5.6 and 5.7 in [6] go through under our weaker hypothesis here.

4.5. $\Xi_{M}=\Xi_{1} * \cdots * \Xi_{s}$, where for each $i, \Xi_{i} \leq \Lambda_{i}$ for some connected component $\Lambda_{i}^{\prime}$ of $\Lambda^{\prime}$, and either $\Xi_{i}=\Lambda_{i}$, or $\Xi_{i}=\Lambda_{i}\left(\leq \gamma_{i}\right) \cup\{\infty\}$ for some $\gamma_{i} \in \Lambda_{i}^{\prime}$.

Proof. By 4.3, $\Xi_{M}$ is a sublattice of $\Lambda$ containing $W_{0}(\leq W)$ for each $W \in \hat{\Xi}_{M}$. But $\Lambda$ is a $D \Delta\left(m_{1}, \ldots, m_{t}\right)$ lattice for some $t>1$ and $m_{i}>2$, so

$$
\Lambda=\Lambda_{1} * \cdots * \Lambda_{t},
$$

where $\Lambda_{i} \cong \Delta\left(m_{i}\right)$ and $\left(\Lambda_{i}^{\prime}: 1 \leq i \leq t\right)$ are the connected components of $\Lambda^{\prime}$. Therefore $\Xi_{M}=\Xi_{1} * \cdots * \Xi_{t}$, where $\Xi_{i}=\Xi_{M} \cap \Lambda_{i}$, and with $\Xi_{i}$ a sublattice of $\Lambda_{i}$ containing $\mathcal{W}_{0}(\leq W)$ for each $W \in \Xi_{i}-\{\infty\}$. Hence the lemma follows from 1.3.

4.6. The following hold:

(1) For each $V \in \hat{\boldsymbol{\Xi}}_{M}, V \tilde{V} \in \hat{\boldsymbol{\Xi}}_{M}$.

(2) The map $\varphi: V \mapsto V \tilde{V}$ is a map of posets from $\hat{\Xi}_{M}$ into $\hat{\Xi}_{M}$ such that

$$
\varphi(V) \geq V .
$$

(3) If $\Xi_{M}$ is disconnected or contains a connected component of $\Lambda^{\prime}$, then

$$
J=\varphi(J) .
$$

(4) If $\Xi_{M}$ contains a connected component $\mathcal{C}$ of $\Lambda^{\prime}$, then $\varphi=1$ on $\mathcal{C}$ and

$$
\varphi(J)=J .
$$


Proof. By 4.4.2, $\tilde{V} J \in \mathcal{W}_{0}$. Next $N_{M}(V)$ permutes the groups $\tilde{V}_{E}, E \in \mathscr{L}$, so $N_{M}(V)$ acts on $\tilde{V}$. If $H_{*} \leq \tilde{V} V$, then $H_{* D} \leq \tilde{V} V \cap D=\tilde{V}(V \cap D)=\tilde{V}$, contradicting $\tilde{V} J \in W_{0}$. Thus $H_{*} \leq \tilde{V} V$, so (1) follows from 2.12.

Suppose $U \in W_{0}$ with $U \leq V$. Then $U_{D} \leq V_{D}$, so for $E \in \mathscr{L}, \tilde{U}_{E} \leq \tilde{V}_{E}$ and hence $\tilde{U} \leq \tilde{V}$. Thus (2) holds.

If $\Xi_{M}$ is disconnected or contains a connected component of $\Lambda^{\prime}$, then there exist $U_{1}, \ldots, U_{r}$ maximal in $\Xi_{M}^{\prime}$ such that $U_{1} \wedge \cdots \wedge U_{r}=J$. By (2), $\varphi\left(U_{i}\right)=U_{i}$ and then

$$
\varphi(J)=\varphi\left(U_{1} \wedge \cdots \wedge U_{r}\right)=\varphi\left(U_{1}\right) \wedge \cdots \wedge \varphi\left(U_{r}\right)=U_{1} \wedge \cdots \wedge U_{r}=J,
$$

establishing (3).

Assume the hypothesis of (4). Then $\mathcal{C}$ is a connected component of $\Xi_{M}$ and $\varphi: \Delta \rightarrow \Delta$, where $\Delta=\ell \cup\{0\}$ by (2) and (3). Now (4) follows from (2) and the dual of 1.1 in [6] applied to $\varphi: \Delta \rightarrow \Delta$.

\section{The case $G$ symmetric and $H$ primitive}

Hypothesis 5.1. Hypothesis 3.1 holds and $G$ is the alternating or symmetric group on a set $\Omega$.

In this section we assume:

Hypothesis 5.2. Hypothesis 5.1 holds and $H$ is primitive on $\Omega$. Set $S=\operatorname{Sym}(\Omega)$ and $A=\operatorname{Alt}(\Omega)$.

We will appeal to the theory of primitive subgroups of $S$ developed in [4] and [5]. In particular from 2.2 and 2.3 in [4], there are five types of primitive groups: affine, semisimple, diagonal, doubled, and complemented primitive groups. Further there are various special classes of semisimple groups: almost simple groups, product indecomposable groups, and octal groups; these classes are not mutually exclusive.

5.3. The following hold:

(1) $H$ is not almost simple.

(2) $J \neq 1$.

(3) For $W \in W_{0}, H W$ is not almost simple.

Proof. By 2.6, (1) is equivalent to (2). Suppose $H$ is almost simple, so that $J=1$. Let $V \in \mathcal{W}_{0}^{\prime}$. Then $M=V H$ is primitive on $\Omega$ by 2.4 in [4], and $H \cap V=J=1$, so $V$ is a normal complement to $H$ in $M$. In particular, $M$ is not almost simple, so by Proposition 2 in [4], $H \cong L_{3}(2),|\Omega|=8$, and $M$ is one of the two maximal 
parabolics of $G \cong L_{4}(2)$ which contain $H$. Thus we get $|\Lambda|=4$, contradicting $\Lambda$ a $D \Delta\left(m_{1}, \ldots, m_{t}\right)$-lattice. This completes the proof of (1) and (2). Then (2) and 2.14 imply (3).

5.4. $|\Omega|$ is not prime.

Proof. If so by 2.2 in [4], $H$ is almost simple or affine. The first case does not hold by 5.3 and the second is out as $H$ is not solvable.

The notion of an ( $m, k)$-product structure on $\Omega$ is defined in Definition 1.5 in [4]. An $(m, k)$-product structure can be thought of as a decomposition of $\Omega$ as a set product of $k$ sets of order $m$, with $m \geq 5$ and $k \geq 2$. The stabilizers of product structures are maximal semisimple primitive subgroups of $S$. There is a partial ordering of product structures defined in Section 5 of [5].

The notion of a pseudo-semisimple subgroup of $S$ is defined in Definition 5.7 of [5]. A pseudo-semisimple group is a primitive group $K$ which preserves a product structure and the set of $K$-invariant product structures has a largest member $\mathcal{F}^{+}(K)$ (also defined in 5.7 of [5]).

5.5. The following hold:

(1) Either $H$ is affine and imprimitive on $F^{*}(H)$, or $H$ is pseudo-semisimple.

(2) Let $V \in \mathcal{W}_{0}$. Then either $H$ is affine, or $H V$ is pseudo-semisimple.

Proof. We first prove (1). By Hypothesis 5.2, $H$ is primitive on $\Omega$. From Definition 5.7 in [5], the primitive group $H$ is pseudo-semisimple unless one of the following holds:

(i) $H$ is almost simple.

(ii) $H$ is affine.

(iii) $H$ is doubled with two components.

(iv) $H$ is strongly diagonal.

By 5.3, (i) does not hold. In case (ii) we may assume $H$ is primitive on $F^{*}(H)$. In this case, and in cases (iii) and (iv), we show

$$
\mathcal{M}(H)=\left\{N_{G}\left(F^{*}(H)\right)\right\},
$$

which contradicts 3.6.2.

If $H$ is affine and primitive on $F^{*}(X)$, then $(*)$ follows from 5.4 and Proposition 4 in [4]. Similarly (*) holds in cases (iii) and (iv) by Propositions 9 and 7 of [4], respectively. This completes the proof of (1).

Finally (2) follows from (1) and 5.8 in [5]. 
5.6. For each $V \in \mathcal{W}_{0}$ :

(1) $F^{*}(H V) \leq V$.

(2) $F^{*}(H V) J \in \mathcal{W}_{0}$.

Proof. We first prove (1). By 5.3.2, $V \neq 1$, so $V$ contains a minimal normal subgroup $E$ of $M:=H V$. By 2.4.1 in [4], $M$ is primitive on $\Omega$, so by 2.2 in [4], either $E=F^{*}(M)$ or $M$ is doubled and $F^{*}(M)=E \times \tilde{E}$, with $\tilde{E} \cong E$, and we may assume the latter, with $V \cap \tilde{E}=1$. Let $M^{*}:=M / V$. As $V \in \mathcal{W}_{0}$, it follows that $F^{*}\left(M^{*}\right)=H_{0}^{*} \cong L$ is a nonabelian simple group. But as $\tilde{E}^{*} \cong \tilde{E}$ is the direct product of simple groups, and as $\tilde{E}^{*} \unlhd H^{*}=M^{*}$, we have

$$
\tilde{E}^{*} \unlhd F^{*}\left(M^{*}\right)=H_{0}^{*},
$$

so $\tilde{E}$ is simple. This is a contradiction as $M$ is pseudo-semisimple by 5.5 .2 , so $\tilde{E}$ has more than one component. This completes the proof of (1). Then (1) and 2.7.2 imply (2).

See 5.7 in [5] for the definition of the product structure $\mathcal{F}^{+}(X)$ of a pseudosemisimple subgroup $X$ of $S$.

5.7. If $V \in \mathcal{W}_{0}$ and $H V$ is pseudo-semisimple, then $\mathcal{F}^{+}(H V)$ is the greatest member of $\mathcal{F}(H V)$.

Proof. This is 5.9.1 in [5].

Notation 5.8. If $V \in \mathcal{W}_{0}$ and $H V$ is pseudo-semisimple, set

$$
M(V):=M\left(\mathscr{F}^{+}(H V)\right),
$$

where $M(\mathcal{F})$ is the stabilizer in $G$ of the product structure $\mathscr{F}$. Set

$$
X(V):=F^{*}(M(V)),
$$

and write $K(V)$ for the kernel of the action of $M(V) \cap \hat{G}$ on the components of $M(V)$. This makes sense by 5.7 .

5.9. Assume $V \in \mathcal{W}_{0}$ and $H V$ is pseudo-semisimple but not affine. Set

$$
D:=F^{*}(H V) \text {. }
$$

Then:

(1) $M(V) \in \mathcal{M}(H V)$.

(2) $F^{*}(H V) \leq X(V)$. 
(3) Assume $H V$ is semisimple and product indecomposable. Then each component $D_{1}$ of $H V$ is contained in a component $X_{1}$ of $X(V)$. Moreover

$$
D_{1}=F^{*}\left(H V \cap X_{1}\right) \text { and } C_{X_{1}}\left(D_{1}\right)=1 .
$$

(4) Assume $H V$ is semisimple and product indecomposable. Then $N_{X(V)}(D) / D$ is solvable and $N_{X(V)}(D) J, X(V) J$, and $K(V) J$ are in $w_{0}$.

Proof. Part (1) follows from the definition of $M(V)$ in 5.8 and Proposition 5 in [4] which says that $M(V) \in \mathcal{M}$. Part (2) follows from 4.3 in [4]; for example in case (2) of 4.3 in [4], the overgroup $M$ is not the stabilizer $M(V)$ of a product structure.

Assume $H V$ is semisimple and product indecomposable. The first remark in (3) follows from Proposition 5 in [4]; the structure of $H V \cap X_{1}$ and the fact that $C_{X_{1}}\left(D_{1}\right)=1$ are consequences of the fact that $\mathcal{F}^{+}(M(V))=\mathcal{F}^{+}(H V)$.

Let $X_{1}, \ldots, X_{r}$ be the components of $X:=X(V)$. Set $F_{i}:=F^{*}\left(H V \cap X_{i}\right)$. We must show that $N_{X}(D) / D$ is solvable. But $D$ is the direct product of the subgroups $F_{i}$ and $N_{X}(D)$ is the direct product of the subgroups $N_{X_{i}}\left(F_{i}\right)$, so it remains to show that $N_{X_{1}}\left(F_{1}\right) / F_{1}$ is solvable. But (3) says that $F_{i}=D_{i}$ is simple, so $\operatorname{Out}_{X_{i}}\left(F_{i}\right)$ is solvable by the Schreier property. Hence $N_{X}(D) / D$ is solvable, and then $X J$ and $N_{X}(D) J$ are in $\mathcal{W}_{0}$ by 2.9. Then we have $K(V) J \in \mathcal{W}_{0}$ by 2.8 as $K(V) / X$ is solvable. This completes the proof of (4).

For $H$ affine with $F^{*}(H)=D$, recall the definition of $\mathscr{D}(H)$ and $\mathscr{F}(\mathscr{D})$ for $\mathscr{D} \in \mathscr{D}(H)$, from 2.6 and 1.6 in [4].

5.10. Assume $H$ is affine, set $D:=F^{*}(H)$, and for $\mathscr{D} \in \mathscr{D}(H)$ let

$$
M(\mathscr{D}):=M(\mathcal{F}(\mathscr{D})) \text { and } X(\mathscr{D}):=F^{*}(M(\mathscr{D})) .
$$

Set $\mathfrak{W}_{1}^{*}:=\left\{V \in \mathfrak{W}_{0}^{*}: V \not N_{G}(D)\right\}$. Then:

(1) $\mathcal{M}(H)=\left\{N_{G}(D), M(\mathscr{D}): \mathscr{D} \in \mathscr{D}(H)\right\}$.

(2) $\mathcal{W}_{1}^{*} \neq \varnothing$.

(3) For $V \in W_{1}^{*}, H V$ is semisimple, product indecomposable, and pseudo-semisimple, and

$$
F^{*}(H V)=X(V),
$$

where $X(V):=X(\mathcal{E}(V))$ for some $\mathcal{E}(V) \in \mathscr{D}(H)$.

(4) $X(V) J=V$.

(5) $N_{X(V)}(D) \leq J$.

(6) $D=F^{*}(J)$. 
Proof. Part (1) follows from 5.4 and Proposition 4 in [4], while part (2) follows from 3.3.

Let $V \in \mathcal{W}_{1}^{*}$ and set $Q:=F^{*}(H V)$. Then $H V \in \mathcal{O}_{G}(H)^{\prime}$, so by 5.4 and Proposition 4 in [4], $D \leq Q$ and either $Q=D$ or $H V$ is semisimple and

$$
\mathcal{F}(H V)=\mathcal{F}(\mathscr{D})
$$

for some $\mathscr{D} \in \mathscr{D}(H)$. As $V \not \leq N_{G}(D)$, the latter holds. Set $\mathcal{E}(V):=\mathscr{D}$.

By 5.6, $\bar{Q} \in W_{0}$ and $\bar{Q} \leq V$. As $D \neq Q, Q \not \leq J$, so $V=\bar{Q}$ as $V \in W_{0}^{*}$.

As $H$ is affine, $|\Omega|$ is a prime power, so $H V$ is product indecomposable (cf. Definition 5.10 in [4]). Hence (cf. Definition 5.7 in [5]) $H V$ is pseudo-semisimple and $\mathcal{F}^{+}(H V)=\mathscr{F}(H V)=\mathscr{F}(D)$, so in the notation of 5.8,

$$
M:=M(\mathscr{D})=M(V) \quad \text { and } \quad X(\mathscr{D})=X(V) .
$$

Set $X:=X(V)$ and $K:=K(V)$. As $X=X(D)$, it follows from 1.6 in [4] that $X=X_{1} \times \cdots \times X_{r}$ is the direct product of its components and $D=D_{1} \times \cdots \times D_{r}$, where $D_{i}=D \cap X_{i}$. By 5.9.4, $\bar{X}$ and $\bar{K}$ are in $\mathcal{W}_{0}$, and by 5.9.2, $Q \leq X$, so $V=$ $\bar{Q} \leq \bar{X}$. As $\mathcal{F}(H V)=\mathscr{D}$ and $H V$ is semisimple and product indecomposable, 5.9.3 says that $Q=Q_{1} \times \cdots \times Q_{r}$, where $Q_{i}=X_{i} \cap Q$ is a component of $Q$.

Suppose $X \neq Q$. Then $V<\bar{X} \leq \bar{K}$, so applying 3.7 to $X, K H$ in the roles of $Y, X$ it follows that $K H=X N_{K H}(Q)$. Also $D_{1} \leq Q_{1}<X_{1}$, so by 3.2.2 in [4], $\left|D_{1}\right|=p$ is prime. As $K H=X N_{K H}(Q)$, we get $K=X_{1} N_{K}\left(Q_{1}\right)$. But now as $\operatorname{Aut}_{K}\left(X_{1}\right) \cong S_{p}, 5.7$ in [14] contradicts $Q_{1}<X_{1}$. Therefore $X=Q$, completing the proof of (3). Further $V=\bar{Q}=\bar{X}$, establishing (4).

Next for $Y \in d_{N_{X}(D)}(H), \bar{Y} \in \mathcal{W}_{0}$ by 2.7.1. Then as $V=\bar{X} \in \mathcal{W}_{0}^{*}$, part (5) follows.

By 5.6, $D \leq J$. As $D=F^{*}(H)$ and $D \leq J \unlhd H$, it follows that $D=F^{*}(J)$, establishing (6).

5.11. Assume $H$ is affine. Then:

(1) There exists a unique $V \in W_{1}^{*}$.

(2) Choose $\mathcal{E}(V)$ as in 5.10.3. Then $\mathcal{E}(V)$ is the unique member of $\mathfrak{D}^{*}(H)$.

Proof. Let $D:=F^{*}(H)$ and $M:=N_{G}(D)$. By 5.10 .2 there exists a $V \in W_{1}^{*}$. Let $\mathcal{E}:=\mathscr{E}(V), X:=X(V), K:=K(V)$, and $P:=N_{X}(D)$. By 5.10.5, $P \leq J$. But from 4.1 in [4], $N_{K}(D)$ is the kernel of the action of $N_{M}(\mathcal{E})$ on $\mathcal{E}$. Thus as $N_{K}(D) / P$ is a 2-group of exponent 2, it follows from 4.7 in [5] that either $\mathscr{E}$ is the unique maximal $P$-invariant member of $\mathscr{D}(H)$, and hence (2) holds, or $\mathscr{D}$ consists of $k$ members of order 5 , and choosing $\mathcal{E}^{\prime} \in \mathscr{D}(H)$ with $\mathcal{E}^{\prime} \not \leq \mathcal{E}, \mathcal{E}^{\prime}$ also has $k$ members of order 5 and $\mathscr{B}:=\mathscr{E} \wedge \mathcal{E}^{\prime}=\left\{B_{1}, \ldots, B_{k / 2}\right\}$ has $k / 2$ members of order 25 . 
Assume the latter case and let $X^{\prime}:=X\left(\mathcal{E}^{\prime}\right)$. Then $P^{\prime}:=N_{X^{\prime}}(D)$ is solvable, so by $2.8, V^{\prime}:=\bar{X}^{\prime} \in W_{0}$. Further arguing as in the proof of 5.10.5, $P^{\prime} \leq J$, so as $P^{\prime}$ is a maximal $H$-invariant subgroup of $X^{\prime}$, we conclude $V^{\prime} \in \mathcal{W}_{0}^{*}$.

Suppose that $k=2$. Then

$$
N_{F^{*}(G)}(D) \cong\left(Z_{4} * \mathrm{SL}_{2}(5)\right) / E_{25}
$$

and $H \leq N_{G}(D)$ acts on $J$ and $X$ with $N_{X}(D) \cong D_{10} \times D_{10}$ contained in $J$, so $N_{G}(J) / J$ is solvable, a contradiction.

Hence $k>2$, so that $\mathscr{B} \in \mathscr{D}(H)$. Moreover there is a third member $\mathcal{E}^{\prime \prime}$ of $\mathscr{D}(H)$ with $\mathcal{B}=\mathcal{E} \wedge \mathcal{E}^{\prime \prime}=\mathcal{E}^{\prime} \wedge \mathcal{E}^{\prime \prime}$, and by symmetry $V^{\prime \prime}=\bar{X}^{\prime \prime} \in \mathcal{W}_{0}$, where $X^{\prime \prime}:=X\left(\mathscr{E}^{\prime \prime}\right)$. Let $Y:=X(\mathscr{B})$ and $Y_{1}$ a component of $Y$. From 5.12 in [5], we have $U \leq Y$ for $U \in\left\{X, X^{\prime}, X^{\prime \prime}\right\}$, and $N_{Y_{1}}(U)$ is the stabilizer in $Y_{1}$ of a regular product structure on $\omega Y_{1}$. In particular $Y_{1}$ contains components $U_{j} \cong A_{5}$ of $U$, $j \in\{1,2\}$, and $Y_{1} \cong A_{25}$ with $N_{Y_{1}}(U) / U_{1} U_{2}$ solvable. Applying 2.8 to $X \cap Y$, $N_{Y}(X), Y$ in the roles of $X_{1}, X_{2}, X_{3}$, we conclude that $\bar{Y} \in \mathcal{W}_{0}$. Then by 2.7, $N_{Y}(U) J \in \mathcal{W}_{0}$ for $U \in\left\{X^{\prime}, X^{\prime \prime}\right\}$. As $N_{Y_{1}}(U)$ is the stabilizer of a regular product structure, it is maximal in $Y_{1}$, so $Y=\left\langle X, N_{Y}(U)\right\rangle$. As $V=\bar{X} \in W_{0}^{*}$ and $N_{Y}\left(X^{\prime}\right) \neq N_{Y}\left(X^{\prime \prime}\right)$, this contradicts 3.4 and 3.7. Therefore (2) holds.

If $V^{\prime} \in \mathcal{W}_{1}^{*}$, then by (2) applied to $V^{\prime}, \mathcal{E}\left(V^{\prime}\right)=\mathscr{E}$, so by $5.10 .4, V^{\prime}=\bar{X}=V$, establishing (1).

\subsection{2. $H$ is not affine.}

Proof. Assume $H$ is affine and set $D:=F^{*}(H), M:=N_{G}(D), M^{*}:=M / D$. By 5.11.1 there is a unique $V \in W_{1}^{*}$. Let $\mathcal{E}:=\mathcal{E}(V)$. As $\Lambda$ is disconnected, there exists $W \in W_{0}^{*}$ with $\mathcal{C}(W) \neq \mathcal{C}(V)$. Then $W \notin W_{1}^{*}$, so $W \leq M$. Set $P:=H W$.

Let $Q:=J \cap X(V)$ and $Q^{\prime}$ the kernel of the action of $N_{M}(\mathcal{E})$ on $\mathcal{E}$. By 4.1 in [4], $Q^{\prime}=N_{K(V)}(D)$. By 5.10.5, $Q \geq N_{X(V)}(D)$, so $Q^{\prime} / Q$ is a 2-group of exponent 2 .

Suppose that $P$ is primitive on $D$. As $H^{*} \leq P^{*}$ with $H^{*}$ not solvable, and as $Q^{\prime} / Q$ is a 2-group of exponent 2, 4.8 and 4.9 in [5] say that $F^{*}\left(P^{*}\right)=P_{0}^{*} Z^{*}$ where $P_{0}^{*}$ is quasisimple and $Z^{*}$ induces scalars on $D$. Thus as

$$
1 \neq Q^{*} \leq W^{*} \unlhd P^{*},
$$

$P_{0}^{*} \leq W^{*}$, so $P^{*} / W^{*}$ is solvable, contradicting $F^{*}(H / W) \cong L$.

Therefore there exists a $\mathscr{D}=\left\{D_{1}, \ldots, D_{m}\right\} \in \mathscr{D}(P)$. Suppose $\mathscr{D}=\mathcal{E}$. Then $P \leq M(V)$ and as $\mathcal{C}(V) \neq \mathcal{C}(W), 3.8$ and 3.9.3.d in [6] say that $V=\theta(W)$ acts on $J$, contrary to $V \in W_{1}^{*}$ and 5.10.6. Thus $\mathscr{D} \neq \mathcal{E}$, so by 5.11.2, $\mathscr{D}<\mathcal{E}$. Let $R$ be the kernel of the action of $N_{M}(\mathscr{D})$ on $\mathscr{D}, R_{P}:=R \cap P$, and $Y:=X(D)$. From 4.1 in [4], $H \cap Y \leq P \cap Y \leq N_{Y}(D) \leq R$. 
As $W \in W_{0}^{*}$, it follows that $H$ is maximal in $P$, so $\mathscr{D}$ is maximal in $\mathcal{E}$. Therefore as $Q^{\prime} / Q$ is a 2-group of exponent 2, 4.10 in [5] says that either $H \cap R$ is solvable or $F^{*}\left(R_{P}^{*}\right)=R_{0}^{*} Z^{*}$ where $Z^{*}$ induces scalars on each $D_{i}, R_{0}^{*}=R_{1}^{*} \cdots R_{m}^{*}$ with $H$ transitive on the $R_{i}^{*}$, and for $1 \leq i \leq m, R_{i}^{*}$ is quasisimple and $R_{i}$ is contained in the $i$ th component of $Y$. As $H \cap Y \leq H \cap R$, in the first case $\bar{Y} \in \mathcal{W}_{0}$ by 2.8. Similarly in the second case, as $1 \neq Q^{*} \leq R_{0}^{*}$, we get $R_{0}=\left\langle Q^{P}\right\rangle \leq W$, so $R /(R \cap W)$ is solvable. Applying 2.8 to $Y \cap W, Y \cap P, Y$ in the role of $X_{1}, X_{2}, X_{3}$, we again conclude that $\bar{Y} \in \mathcal{W}_{0}$. As $\mathscr{D}<\mathcal{E}, \mathcal{F}(D)<\mathcal{F}(\mathcal{E})$, so $X(V)<Y$ by 5.12.1 in [5]. But now $\mathcal{C}(V)=\mathcal{C}(\bar{Y})$, and arguing as in the previous paragraph, $\mathcal{C}(\bar{Y})=\mathcal{C}(W)$, contrary to the choice of $W$.

\subsection{If $H$ is semisimple, then $H$ is not octal.}

Proof. See 4.2 in [4] for the definition of an octal semisimple group. Assume $H$ is octal, and set $X:=X(J), M:=M(J)$, and $K:=K(J)$. By 5.9.4, $\bar{X}$ and $\bar{K}$ are in $W_{0}$. Let $D_{1}$ be a component of $H$. As $H$ is octal, from 4.2 in [4], it follows that $D_{1} \cong \operatorname{Aut}_{H}\left(D_{1}\right) \cong L_{3}(2)$ and $\Gamma_{1}:=\omega D_{1}$ is of order 8. By 5.9.3, $D_{1}$ is contained in a component $X_{1}$ of $X$. As $\left|\Gamma_{1}\right|=8, X_{1} \cong A_{8} \cong L_{4}$ (2). Now (cf. Proposition 2 in [4]) $X \cap J=F^{*}(H)=D$ and $\mathcal{V}_{X}(H)=\left\{D, Y_{1}, Y_{2}, X\right\}$, where $Y_{i}$ is affine with $Y_{i, 1}=Y_{i} \cap X_{1} \cong L_{3}(2) / E_{8}$ a maximal parabolic of $X_{1}$. By 2.7, we have $\bar{Y}_{i} \in \mathcal{W}_{0}$. As $Y_{1} \cap Y_{2}=D=X \cap J$, it follows from 3.4 that $\bar{Y}_{i} \in \mathcal{W}_{0}^{*}$ and $\bar{X}=\bar{Y}_{1} \vee \bar{Y}_{2}$. But $X<K$, and as $\operatorname{Aut}_{H}\left(D_{1}\right)=D_{1}, N_{K}(D)=D$. As $K / X$ is solvable, $\bar{K} \in \mathcal{W}_{0}$ by 2.8. As $N_{K}(D)=D=X \cap J$, 3.7.4 supplies a contradiction.

5.14. We have $\mathcal{M}(H)=\{M(\mathcal{F}): \mathcal{F} \in \mathcal{F}(H)\}$, and $\mathcal{F}^{+}(H)$ is the greatest member of $\mathcal{F}(H)$.

Proof. By 5.12, $H$ is not affine, so by 5.5.2, $H$ is pseudo-semisimple. By 5.13, $H$ is not octal semisimple. Thus the lemma follows from 5.17 in [5] and 5.7.

\subsection{The following hold:}

(1) $H$ is not doubled, complemented, or diagonal.

(2) If $H$ is semisimple, then $H$ is product indecomposable.

Proof. See 2.3 in [4] for the definition of doubled, complemented, and diagonal primitive groups. See 5.10 in [4] for the definition of product decomposable primitive groups. Assume $H$ is a counterexample to (1) or (2), and set $D:=F^{*}(H)$, $X:=X(J), M:=M(J)$, and $\mathcal{F}:=\mathscr{F}^{+}(H)$. By 3.3 there exists $V \in W_{0}^{*}$ with $V \not \leq M$. By 5.12 and 5.5.2, $H V$ is pseudo-semisimple, so $\mathcal{F}^{+}(H V)$ exists by 5.7. 
Then by $5.14, \mathcal{F}^{+}(H V)<\mathscr{F}$, so by 5.14 in [5] we get $X \leq X(H V)=: Y$. As $V \not \leq M=N_{G}(X), X \neq Y$, and hence $\mathcal{F} \neq \mathscr{F}^{+}(H V)$.

Claim $H V$ is semisimple. If $H$ is semisimple, then this is a consequence of Proposition 5 in [4], and the fact that $H$ is not octal by 5.13. So we may assume $H$ is doubled, complemented, or diagonal. If $D=F^{*}(H V)$, or $H$ is complemented and $F^{*}(H V)=D C_{G}(D)$, then from Definition 5.7 in [5] and Notation 2.6 in [4], $\mathscr{F}=\mathcal{F}^{+}(H)=\mathscr{F}(H)=\mathscr{F}(H V)=\mathscr{F}^{+}(H V)$, contrary to the remark at the end of paragraph one of the proof. Thus by Propositions 7, 9, and 11 in [4], $H V$ is semisimple. This completes the proof of the claim.

We next claim that $F^{*}(H V)=Y$. Assume otherwise. By 5.8.3 in [4], the parameter $b$ of Notation 5.2 in [4] is 1 or 2. Suppose $b=2$. Then by 5.9 .3 and by Definition 5.10 and 5.8.4 in [4], $H$ is product decomposable, while by 5.11 in [4], $\mathcal{F}^{+}(H V)=\mathscr{F}$, contrary to an earlier remark. Thus $b=1$. As $H<H V$, we conclude from 5.5.5 in [4] that $H$ is semisimple and the parameter $s$ of Notation 5.2 in [4] is equal to 1 . As $b=1$, a component $D_{1}$ of $D$ is contained in a component $L_{1}$ of $H V$, and then as $s=1, D_{1}$ is transitive on $\Gamma_{1}:=\omega L_{1}$. As $H$ is semisimple and a counterexample to (1) or (2), $H$ is product decomposable. Now by the Main Theorem of [12], either $N_{\mathrm{Sym}\left(\Gamma_{1}\right)}\left(D_{1}\right)$ is the unique maximal overgroup in $\operatorname{Sym}\left(\Gamma_{1}\right)$ of $D_{1}$, so that $L_{1}=D_{1}$, or $D_{1} \cong \operatorname{Sp}_{4}(q)$ and $L_{1} \cong \operatorname{Sp}_{4 e}\left(q^{1 / e}\right)$ for some $e$. In the first case $D=F^{*}(H V)$ so $H V$ is also product decomposable and $\mathcal{F}^{+}(H V)=\mathcal{F}$, a contradiction. The second case is impossible as some element of $H$ induces an automorphism on $D_{1}$ nontrivial on the Dynkin diagram of $D_{1}$, and such automorphisms do not lift to $L_{1}$. This completes the proof of the second claim.

By the second claim and 5.6.2, $\bar{Y} \in \mathcal{W}_{0}$. Then $\bar{X} \in \mathcal{W}_{0}$ by 2.7. Now as $X<Y$, $Y$ does not act on $X$. As $M$ is the stabilizer of a product structure, $M$ is semisimple and product indecomposable. Then as $H$ is not, $D<X$, so $X$ does not act on $D$. Then as $J$ does act on $D$ and $X$, it follows that $J=\bar{D} \neq \bar{X} \neq \bar{Y}$. Then $V \in W_{0}^{*}$ and $J<\bar{X}<\bar{Y}=V$, so we have a contradiction.

5.16. $H$ is semisimple, product indecomposable, and not octal.

Proof. By 5.12, $H$ is not affine, so by 5.15.1, $H$ is semisimple. Then by 5.13, $H$ is not octal, and by 5.15.2, $H$ is product indecomposable, completing the proof.

Notation 5.17. Adopt Notation 5.8 , and let $\mathscr{F}:=\mathscr{F}^{+}(H)$ and $X:=X(J)$. Let $\Xi:=\mathscr{F}(H)-\{\mathcal{F}\}$ and $\Xi^{*}$ the maximal members of $\Xi$. Set

$$
\mathcal{W}_{1}^{*}:=\left\{V \in \mathcal{W}_{0}^{*}: \mathcal{F}^{+}(H V) \in \Xi^{*}\right\} .
$$

Let $I:=\{1, \ldots, r\}$ and $\mathcal{X}:=\left\{X_{i}: i \in I\right\}$ be the set of components of $X$. Set $m:=\left|\omega X_{1}\right|$ for $\omega \in \Omega$. Thus $\mathcal{F}$ is a regular $(m, r)$-product structure on $\Omega$. 
Represent $N_{S}(\mathscr{F})$ on $I$ so that the map $\varphi: i \mapsto X_{i}$ is an equivalence of that representation with the representation of $N_{S}(\mathscr{F})$ on $\mathcal{X}$ via conjugation. For given $Y \leq N_{S}(\mathcal{F})$, write $Y^{I}$ for the image of $Y$ in $\operatorname{Sym}(I)$ under this representation.

5.18. The following hold:

(1) $X=F^{*}(H)=F^{*}(J)$.

(2) $K(J) \leq J$.

(3) For $V \in \mathfrak{W}_{0}, H V$ is semisimple, product indecomposable, and not octal; moreover $X \leq X(V)$, and $K(J) \leq K(V)$.

(4) $(M(J) \cap A)^{I}=\operatorname{Sym}(I)$.

Proof. Let $V \in \mathcal{W}_{0}$. By 5.16 and Proposition 5 in [4], $H V$ is semisimple and product indecomposable but not octal. This allows us to verify the hypothesis of 2.10 with $M(V)$ and $K(V)$ defined in 5.8. Then we appeal to that lemma.

Hypothesis (a) of 2.10 follows from 5.6.1. As $F^{*}(M(V))$ is perfect, we have $F^{*}(M(V)) \leq F^{*}(G)$. By 5.9.1, $M(V) \in \mathcal{M}(H V)$, while by 5.9.2, $F^{*}(H(V)) \leq$ $X(V)$. Then by 5.9.4, the remaining condition in hypothesis (b) of 2.10 is satisfied.

As $H$ is semisimple, $F^{*}(H)$ is the unique member of the set $\mathcal{X}$ of 2.10.2. As $M(J)=M\left(\mathcal{F}^{+}(H)\right)$, we get $N_{G}\left(F^{*}(H)\right) \leq M(J)$. By 5.14, and by 5.14 in [5], $X \leq X(V)$, so hypothesis (c) of 2.10 is satisfied, and the second statement in (3) holds. Hence as $\Lambda$ is disconnected, $X \leq J$ by 2.10.2. Then as $J \unlhd H \leq M(J)$ and $X=E(X)=F^{*}(M(J))$, (1) holds.

By construction, $X(V) \leq K(V) \unlhd M(V)$ with $K(V) \leq \hat{G}$, and $K(V) / X(V)$ is a 2-group. As $\mathcal{F} \leq \mathscr{F}(H V), K(J) \leq K(V)$, completing the proof of (3). Now (2) follows from 2.10.3.

By (2), $H \cap K(J) \leq J$, so as $H / J$ is not solvable, $r>2$. Thus (4) follows from 5.10 .3 in [5].

5.19. Let $V \in \mathcal{W}_{0}$. Then:

(1) $H V$ is semisimple, product indecomposable, and not octal.

(2) $X \leq X(V)=F^{*}(H V)$.

(3) If $V \in W_{0}^{*}$, then either

(i) $X(V)=X$ and $H$ is maximal in $H V$, or

(ii) $V=X(V) J, V \in W_{1}^{*}$, and $N_{X(V)}(X) \leq J$.

(4) $K(V) J \in \mathcal{W}_{0}$. 
Proof. By 5.18.1, $X=F^{*}(H)$. For $P \leq G$ let $m(P):=|\omega P|$. A component $X_{1}$ of $X$ is isomorphic to the alternating group of degree $m:=m\left(X_{1}\right)$.

We first prove (1) and (2). By 5.10.3, $H V$ is semisimple and product indecomposable, but not octal, and by Proposition 5 in [4], $X_{1}$ is contained in a component $Y_{1}$ of $Y:=F^{*}(H V)$. By 5.9.3, $Y_{1}$ is contained in a component $D_{1}$ of $X(V)$ and $Y_{1}=F^{*}(H V) \cap D_{1}$, so $m\left(Y_{1}\right)=m\left(D_{1}\right)$. Let $s$ be the number of components of $H$ contained in $Y_{1}$; this is the parameter defined in Notation 5.2 of [4]. By 5.5.5 in [4], either $Y_{1} \cong A_{m\left(Y_{1}\right)}$, or $s=1$. In the former case as $m\left(Y_{1}\right)=m\left(D_{1}\right), Y_{1}$ is a component of $X(V)$ and statement (1) holds, so assume $s=1$. Then $m\left(Y_{1}\right)=m$, and $X_{1} \leq Y_{1} \leq D_{1}$, with $D_{1} \cong A_{m\left(Y_{1}\right)}$, so as $X_{1} \cong A_{m}, X_{1}=Y_{1}=D_{1}$. This completes the proof of (1) and (2).

We next prove (3), so assume $V \in W_{0}^{*}$.

By 2.12.1 in [6], $H$ is maximal in $H V$. Thus (3i) holds if $X=X(V)$, so we may assume $X(V) \neq X$. By (2) and 5.6, $X(V) \leq V$ and $V^{\prime}:=\overline{X(V)} \in \mathcal{W}_{0}$, so that $V^{\prime} \leq V$. Further if $\mathcal{F}^{+}(H V) \leq \mathscr{D}<\mathcal{F}$, then by 5.14 in [5], $X(\mathscr{D}) \leq X(V)$, so $V^{\prime \prime}:=\overline{X(\mathscr{D})} \in \mathcal{W}_{0}$ by 2.7. Thus as $V \in \mathcal{W}_{0}^{*}, V^{\prime}=V=V^{\prime \prime}$, so $V=\overline{X(V)}$ and $\mathcal{F}^{+}(H V) \in \Xi^{*}$. Hence $V \in \mathcal{W}_{1}^{*}$. Further by $2.7, N_{X(V)}(X) J \in \mathcal{W}_{0}$, so as $V \in W_{0}^{*}, N_{X(V)}(X) \leq J$. This shows that if $V \in W_{0}^{*}$ and (3i) fails, then (3ii) holds, completing the proof of (3).

As $\overline{X(V)} \in \mathcal{W}_{0}$ and $K(V) / X(V)$ is solvable, (4) follows from 2.8 .

5.20. $w_{1}^{*} \neq \varnothing$.

Proof. Assume otherwise. Then by 5.19.3, $W_{0}^{*} \subseteq M(J)$, contrary to 3.3.

5.21. Let $V \in W_{1}^{*}$. Let $\mathcal{X}(V)$ be the set of components of $X(V)$, and for $Y \in \mathcal{X}(V)$ $\operatorname{set} \sigma(Y)=\left\{i \in I: X_{i} \leq Y\right\}$ and $k=k(V):=|\sigma(Y)|$. Set $P(V):=N_{K(V)}(X)$. Then:

(1) $\Sigma=\Sigma(V):=\{\sigma(Y): Y \in \mathcal{X}(V)\}$ is an $H$-invariant partition of I such that $N_{M(J)}(\Sigma)=M(J) \cap M(V)$.

(2) $P(V)^{I}$ is the kernel $K(\Sigma)$ of the action of $N_{\mathrm{Sym}(I)}(\Sigma)$ on $\Sigma$.

(3) Either $N_{X(V)}(X)^{I}=K(\Sigma)$ or $k=2, m \equiv 2 \bmod 4, N_{X(V)}(X)=K(J)$, and the involution $\tau=\tau(V) \in \operatorname{Sym}(I)$ with cycles $(i, j)$ for $\{i, j\} \in \Sigma$ is in $J^{I}$.

(4) Let $U \in \mathcal{W}_{0}$ and set $Q:=N_{H U}(X)^{I}$. Assume either

(a) $P(V) \leq U$, or

(b) $N_{X(V)}(X)^{I}=K(\Sigma)$.

Then $Q$ is imprimitive on $I$, and for each nontrivial $Q$-invariant partition $\Gamma$ of $I, \Gamma \leq \Sigma$.

(5) $\mathcal{W}_{1}^{*}=\{V\}$. 
Proof. Set $T:=N_{S}(X(V)) \cap N_{S}(X)$. Visibly $\Sigma$ is a $T$-invariant partition of $I$. By 5.14, $\mathcal{F}^{+}(H V) \leq \mathcal{F}$, so by 5.12.2 in [5], $T$ is the stabilizer in $N_{S}(X)$ of a nontrivial partition $\Sigma^{\prime}$ of $I$. By 1.8 in [4], $N_{S}(\mathscr{F})^{I}=\operatorname{Sym}(I)$, so $\Sigma^{\prime}$ is the unique nontrivial $T$-invariant partition of $I$, and hence $\Sigma=\Sigma^{\prime}$ and

$$
N_{M(J)}(\Sigma)=M(J) \cap M(V),
$$

establishing (1).

Next $P(V)$ acts on each block of $\Sigma$ so $P(V)^{I} \leq K(\Sigma)$. On the other hand by (1),

$$
N_{M(J)}(\Sigma)=M(J) \cap M(V),
$$

so $M(J)_{\Sigma}$ is the kernel of the action of $M(J) \cap M(V)$ on $\Sigma$. Then (2) follows from 5.18.4.

By 5.12.4 in [5], $T$ is the subgroup of $N_{S}(X(V))$ permuting the set $\mathcal{C}$ of product structures defined in that lemma. Hence for $Y \in \mathcal{X}(V), N_{Y}(X)$ is the stabilizer of the $(m, k)$-product structure on the $m^{k}$-set permuted by $Y$. Then all but the last remark in part (3) follow from 5.10.3 in [5], which says the $Y^{\alpha}=\operatorname{Sym}(Y)$, where $\alpha:=\left\{X_{i}: X_{i} \leq Y\right\}$, unless the exceptional case in (3) holds. Further in that event, there is $t \in K(V)$ with $t^{I}=\tau$ the involution in (3). Set $B:=\langle t\rangle X(V)$. Then $B$ is $H$-invariant, so by 5.19.4 and 2.7, $\bar{B} \in \mathcal{W}_{0}$. Further if $B \neq(B \cap J) X(V)$, then by 3.7, $|\bar{B}: \overline{X(V)}|=2$, contrary to 3.5.2. Thus we may take $t \in J$, completing the proof of (3).

Assume the hypothesis and setup of (4). As $V \in W_{1}^{*}, N_{X(V)}(X) \leq J$ by 5.19.3. In case (4b), $K(\Sigma)=N_{X(V)}(X)^{I} \leq J^{I} \leq U^{I}$, while in case (4a), $P(V) \leq U$ by hypothesis, and by $(2), K(\Sigma)=P(V)^{I}$. Therefore in either case,

$$
K(\Sigma) \leq U^{I} \leq Q
$$

Thus if $Q$ is primitive on $I$, then by 3.2.1 in [8], $Q$ is the symmetric group on $I$ and $Q=U^{I}$. This is impossible as the kernel $K(J)$ of the action of $M(J)$ on $I$ is contained in $J$ by 5.18.2, and $H \leq N_{H U}(X)$ with $H \cap U=J$ and $F^{*}(H / J) \cong L$. Therefore $Q$ is imprimitive on $I$.

Pick $\Gamma$ as in (4). As $K(\Sigma) \leq Q^{I}, 3.2 .2$ in [8] shows that $\Gamma \leq \Sigma$, completing the proof of (4).

Suppose $V^{\prime} \in W_{1}^{*}-\{V\}$. Then by (3) and (4) applied to $V^{\prime}, \Sigma^{\prime}=\Sigma\left(V^{\prime}\right)$ in the role of $U, \Gamma$, either $k=2$ or $\Sigma^{\prime} \leq \Sigma$. Assume $k>2$. If $k\left(V^{\prime}\right)=2$ then $\Sigma^{\prime}$ is a maximal partition of $I$, so $\Sigma^{\prime}=\Sigma$. On the other hand if $k\left(V^{\prime}\right)>2$, then by symmetry between $V$ and $V^{\prime}, \Sigma \leq \Sigma^{\prime}$, so again $\Sigma=\Sigma^{\prime}$. Thus in any event, $\mathscr{F}^{+}(H V)=\mathscr{F}^{+}\left(H V^{\prime}\right)$ by (1) and 5.12.2 in [5], and then $V=X\left(\mathscr{F}^{+}(H V)\right)=$ $X\left(\mathscr{F}^{+}\left(H V^{\prime}\right)\right)=V^{\prime}$, a contradiction. 
So we may assume $k=2$, and then by symmetry also $k\left(V^{\prime}\right)=2$. Now we have $\left[\tau, \tau\left(V^{\prime}\right)\right]=1$ and $H^{I}$ centralizes $E^{I}=\left\langle\tau, \tau\left(V^{\prime}\right)\right\rangle$, so $H$ preserves the partition $\Phi$ of $I$ consisting of the orbits of $E^{I}$ on $I$. Moreover the kernel $K(\Phi)$ of the action of $M(J)^{I}=\operatorname{Sym}(I)$ on $\Phi$ is a direct product $K(\Phi)=K_{1} \times \cdots \times K_{S}$, where $K_{i} \cong S_{4}, K_{i}=\left\langle K_{i} \cap P(V)^{I}, K_{i} \cap P\left(V^{\prime}\right)^{I}\right\rangle, K_{i} \cap P(V)^{I} \cong K_{i} \cap P\left(V^{\prime}\right)^{I} \cong E_{4}$. Let $R$ be the preimage of $K(\Phi)$ in $M(J)$. Then $R / K(J)$ is solvable, so $\bar{R} \in \mathcal{W}_{0}$ by 2.8. Also $\bar{P}(V)$ and $\bar{P}\left(V^{\prime}\right) \in \mathcal{W}_{0}$. Finally $N_{H}\left(K_{1}\right)$ acts on the third 4-subgroup $\bar{P}_{1}^{I}$ of $K_{1}$ generated by transpositions, and hence on its preimage $P_{1}$ in $M(J)$, and on $P=\left\langle P_{1}^{H}\right\rangle$. Thus $\bar{P} \in \mathcal{W}_{0}$ by 2.7 . Next

$$
P(V)^{I} \cap P\left(V^{\prime}\right)^{I}=O_{2}(K(\Phi))=N_{K(\Phi)}(\Sigma) \cap N_{K(\Phi)}\left(\Sigma^{\prime}\right),
$$

so $J \cap R$ is contained in the preimage $R_{0}$ of $O_{2}(K(\Phi))$ in $R$. As

$$
P(V) \cap P\left(V^{\prime}\right)=P(V) \cap P=P\left(V^{\prime}\right) \cap P=R_{0},
$$

this is contrary to 3.7. Thus (5) holds.

5.22. Let $V \in W_{1}^{*}$ and set $P(V):=N_{K(V)}(X)$. Assume $P(V) \leq U \in W_{0}$ with $U \leq M(J)$, and set $Q:=(H U)^{I}$. Then there exists $\mathscr{D} \in \mathcal{F}(H U)$ such that

$$
\mathscr{D} \leq \mathscr{F}^{+}(H V) \text { and } X(V) \leq X(\mathscr{D})
$$

Proof. By hypothesis,

$$
P(V) \leq U \leq M(J),
$$

so we can form $U^{I}$, and $P(V)^{I} \leq U^{I}$. Define $\Sigma=\Sigma(V)$ as in 5.21. By 5.21.4, there is a nontrivial $Q$-invariant partition $\Gamma$ of $I$ and $\Gamma \leq \Sigma$. As $\Gamma$ is $H U$-invariant, it determines $\mathscr{D}:=\mathscr{F}(H, \Gamma) \in \mathcal{F}(H U)$ by 2.6 in [4]. As $\Gamma \leq \Sigma$, it follows that $\mathscr{D} \leq \mathcal{F}^{+}(H V)$, and hence $X(V) \leq X(D)$ by 5.14 in [5]. This completes the proof of the lemma.

Theorem 5.23. Assume Hypothesis 5.1. Then $H$ is not primitive on $\Omega$.

Proof. Assume otherwise. Then Hypothesis 5.2 is satisfied, so we can appeal to the results in this section. In particular, appealing to 5.20 and 5.21.5, there is a unique $V \in W_{1}^{*}$. Let $\mathcal{C}=\mathcal{C}(V)$ be the connected component of $\Lambda$ containing $V$ and adopt the notation of 3.4. Set $\alpha:=\mathcal{C}^{*}-\{V\}$ and $U:=L_{\alpha}$.

As $W_{1}^{*}=\{V\}$, each $W \in W_{0}^{*}-\{V\}$ satisfies 5.19.3.i. In particular, we have $X(W)=X$, so by 5.19.2, $X=F^{*}(H W)$, so $W \leq M(J)$. Thus $U \leq M(J)$.

On the other hand as $V \in W_{1}^{*}$, it follows that $V$ satisfies 5.19.3.ii, so $V=\overline{X(V)}$ and $N_{X(V)}(X) \leq J$. As in 5.22, set $P(V):=N_{K(V)}(X)$. Then

$$
P(V) \cap X(V)=N_{X(V)}(X) \leq J,
$$


so $P(V) \leq U$ by 3.4. Thus the hypotheses of 5.22 are satisfied, so we may choose $\mathscr{D}$ and $D:=X(\mathscr{D})$ as in that lemma. Thus $\mathscr{D} \in \mathcal{F}(H U)$ so $U \leq M(\mathscr{D})$, while $X(V) \leq D$ by 5.22 so $V=\overline{X(V)} \leq M(D)$. Therefore $\ell^{*}$ acts on $D$, contrary to 3.8. This completes the proof of the theorem.

\section{The transitive but imprimitive case}

In this section we assume:

Hypothesis 6.1. Hypothesis 5.1 holds with $H$ transitive but not primitive on $\Omega$. Set

$$
\begin{aligned}
& \mathcal{P}_{1}=\mathcal{P}_{1}(H):=\left\{\Gamma \in \mathcal{P}^{\prime}(H): H_{*} \leq G_{\Gamma}\right\}, \\
& \mathcal{P}_{2}=\mathcal{P}_{2}(H):=\mathcal{P}^{\prime}(H)-\mathcal{P}_{1}(H) .
\end{aligned}
$$

For $\Gamma \in \mathcal{P}:=\mathcal{P}(\Omega)$, set $M(\Gamma):=N_{G}(\Gamma)$ and $D(\Gamma):=K_{+}(\Gamma)$, except when $\Gamma$ has blocks of size 2 , where we set $D(\Gamma):=\hat{G}_{\Gamma}$.

6.2. The following hold:

(1) $\mathcal{P}(H)$ is a sublattice of $\mathcal{P}$.

(2) Pick $\omega \in \Omega$, and for $U \in \mathcal{O}_{H}\left(H_{\omega}\right)$, set $\gamma(U):=\omega U$. Then the map

$$
U \mapsto \gamma(U)^{H}
$$

is an isomorphism of the dual of the poset $\mathcal{O}_{H}\left(H_{\omega}\right)$ with the poset $\mathcal{P}(H)$.

Proof. If $H$ acts on $\Gamma$ and $\Sigma$ in $\mathcal{P}(\Omega)$, then it acts on $\Gamma \vee \Sigma$ and $\Gamma \wedge \Sigma$, so (1) holds. See 5.18 in [1] for (2).

6.3. Suppose $\Gamma \in \mathcal{P}^{\prime}(H)$. Then:

(1) $\Gamma \in \mathcal{P}_{2}$ if and only if $H_{*} \leq \pm D(\Gamma)$ if and only if $H_{*} \leq \bar{D}(\Gamma)$.

(2) If $\Gamma$ has blocks of size $k \leq 4$, then $\Gamma \in \mathcal{P}_{2}(H)$.

Proof. If $\Gamma \in \mathcal{P}_{2}$, then $H_{*} \not \leq K=G_{\Gamma}$, so as $D:=D(\Gamma) \leq K, H_{*} \not \leq D$. On the other hand, if $H_{*} \not \leq D$, then $H_{*} \not \leq K$ as $K / D$ is solvable and $H_{*}=H_{*}^{\infty}$. Now (1) follows from 2.12 .

Under the hypothesis of (2), $K$ is solvable, so $H_{*} \not \leq K$. Thus (2) holds.

6.4. Suppose $\Gamma \in \mathcal{P}_{2}(H)$. Then:

(1) $\hat{G}_{\Gamma} J$ and $\bar{D}(\Gamma)$ are in $W_{0}$.

(2) If $\Gamma \leq \Sigma \in \mathcal{P}^{\prime}(H)$, then $\Sigma \in \mathcal{P}_{2}(H), \hat{G}_{\Sigma} \leq \hat{G}_{\Gamma}$, and if $|\Sigma| \neq n / 2$, then we have $D(\Sigma) \leq D(\Gamma)$. 
(3) Let $Y \in \mathcal{O}_{G}(D(\Gamma) H)-\{G\}$. Then $Y$ is imprimitive on $\Omega$, $\mathcal{P}^{\prime}(Y)$ has a greatest member $\Gamma_{Y}, \Gamma_{Y} \leq \Gamma, D\left(\Gamma_{Y}\right) \leq Y$, and $D(\Gamma) \leq \hat{G}_{\Gamma_{Y}}$.

(4) If $D(\Gamma) \leq Y=W H$ for some $W \in \mathcal{W}_{0}$, then we have $D(\Gamma) \leq W \geq D\left(\Gamma_{Y}\right)$, so $\Gamma_{Y} \in \mathcal{P}_{2}(H)$.

Proof. Part (1) follows from 2.12. Assume $\Gamma \leq \Sigma$. Then by 3.4.2 in [8], $S_{\Sigma} \leq S_{\Gamma}$, so (2) follows.

Let $k$ be the size of a block in $\Gamma$. We next prove (3). If $k>2$, then (3) follows from 3.2 in [8]. Thus we may take $k=2$. As $H$ is not solvable, neither is $M(\Gamma)$, so $n>8$. Hence by 4.5 in [8], $Y$ is imprimitive on $\Omega$. By 4.4.3 in [8], $\Sigma \leq \Gamma$ for each $\Sigma \in \mathcal{P}^{\prime}(Y)$, so $D(\Gamma) \leq \hat{G}_{\Sigma}$. Thus as $\mathcal{P}(Y)$ is a sublattice of $\mathcal{P}, \mathcal{P}^{\prime}(Y)$ has a greatest member $\Gamma_{Y}$. Let $\sigma \in \Sigma:=\Gamma_{Y}$. By maximality of $\Sigma, Y^{\sigma}$ is primitive. Further $D(\Gamma)^{\sigma}=\operatorname{Sym}(\sigma)_{\Gamma_{\sigma}}$, so by 3.2 in [8], $Y^{\sigma}=\operatorname{Sym}(\sigma)$. Then

$$
\operatorname{Alt}(\sigma)=X^{\sigma}, \quad \text { where } X:=\left\langle D(\Gamma)_{\Omega-\sigma}^{N_{Y}(\sigma)}\right\rangle \leq Y_{\Omega-\sigma},
$$

so $D(\Sigma) \leq Y$. Hence (3) also holds when $k=2$.

Finally assume the hypothesis of (4), and let $\Sigma \in\left\{\Gamma, \Gamma_{Y}\right\}, r$ the size of a block of $\Sigma$, and $E:=D(\Sigma)$. If $\Sigma=\Gamma$, then $E \leq Y$ by hypothesis, while if $\Sigma=\Gamma_{Y}$, then $E \leq Y$ by part (3). If $r \leq 4$, then $\Sigma \in \mathcal{P}_{2}(H)$ by 6.3.2, and $E$ is solvable so $W E \in \mathcal{W}_{0}$ by 2.13 . Hence $H_{*} \not \subset E$ by 2.12 , so as $H_{*} W / W=F^{*}(Y / W)$, it follows that $E \leq W$, and (4) holds in this case.

Thus we may take $r>4$. Then $E$ is a minimal normal subgroup of $E H=Y$, so either $E \leq W$ or $E \cap W=1$. As $F^{*}(Y / W)=H_{*} W / W$ is simple, but $E$ is not, the latter is impossible, so $E \leq W$. Thus $H_{*} \not \leq E$, so $\Sigma \in \mathcal{P}_{2}(H)$ by 6.3.1, completing the proof of (4).

6.5. Suppose $\mathcal{P}_{1}(H) \neq \varnothing$. Then:

(1) $\mathcal{P}_{1}(H)$ has a greatest member $\Gamma_{1}(H)$.

(2) $\Gamma_{1}(H)$ is the set of orbits of $H_{*}$ on $\Omega$.

(3) If $\Sigma \in \mathcal{P}^{\prime}(H)$ with $\Sigma \leq \Gamma_{1}(H)$, then $\Sigma \in \mathcal{P}_{1}(H)$.

(4) Let $W \in \mathcal{W}_{0}, Y:=W H$, and suppose $\Sigma \in \mathcal{P}_{1}(H) \cap \mathcal{P}(Y)$ such that $W_{\Sigma} \neq 1$. Let $\sigma \in \Sigma$. Then $Y^{\sigma}$ is not almost simple.

Proof. Let $\mathcal{O}$ be the set of orbits of $H_{*}$ and $\Gamma \in \mathcal{P}_{1}(H)$. As $H$ is transitive on $\Omega$ and $H_{*} \unlhd H$, it follows that $\mathcal{O} \in \mathcal{P}(H)$. Also $H_{*} \leq \hat{G}_{\Gamma}$, so $\Gamma \leq \mathcal{O}$, and in particular $\mathcal{O} \in \mathcal{P}^{\prime}(H)$. By construction, $H_{*} \leq G_{\mathcal{O}}$, so $\mathcal{O} \in \mathcal{P}_{1}$. Thus (1) and (2) hold.

Assume the hypothesis of (3). Then $\Sigma \leq \mathcal{O}$, so each orbit of $H_{*}$ is contained in a block of $\Sigma$, and hence $H_{*} \leq G_{\Sigma}$, so (3) follows. 
Assume the hypothesis of (4) with $\Sigma=\left\{\sigma_{1}, \ldots, \sigma_{m}\right\}$ and $\sigma=\sigma_{1}$. Then

$$
D:=D(\Sigma)=D_{1} \times \cdots \times D_{m},
$$

where $D_{i}=A_{\Omega-\sigma_{i}}$. Let $\pi_{i}: D \rightarrow D_{i}$ be the $i$ th projection. Assume $Y^{\sigma}$ is almost simple and set $X:=Y_{\Sigma}^{\infty}$. As $\Sigma \in \mathcal{P}_{1}(H), H_{*} \leq X$ and $X \pi_{i}=X^{\sigma_{i}}=F^{*}\left(Y^{\sigma_{i}}\right)$ is a nonabelian simple group for $1 \leq i \leq m$. Thus by 1.4 in [7], there is $\Gamma \leq \Sigma$ such that

$$
X=\prod_{\gamma \in \Gamma} X_{\gamma},
$$

where $X_{\gamma}$ is a full diagonal subgroup of $D_{\gamma}=\prod_{i \in \gamma} X \pi_{i}$. Hence $\left\{X_{\gamma}: \gamma \in \Gamma\right\}$ is the set of components of $X$, and as $H$ is transitive on $\Gamma, X$ is a minimal normal subgroup of $H X$. But $W_{\Sigma} \neq 1$, so $1 \neq W_{\Sigma}^{\sigma} \unlhd Y^{\sigma}$, and hence

$$
X^{\sigma}=F^{*}\left(Y^{\sigma}\right) \leq W_{\Sigma}^{\sigma},
$$

so that $W_{\Sigma}^{\infty} \neq 1$. Then $W_{\Sigma}^{\infty}$ is a nontrivial normal subgroup of $H X$ contained in $X$, so $X \leq W$ by minimality of $X$. But now $H_{*} \leq X \leq W$, a contradiction.

6.6. Set $\mathcal{P}_{J}(H):=\left\{\Gamma \in \mathcal{P}^{\prime}(H): D(\Gamma) \leq J\right\}$. Assume $\mathcal{P}_{J}(H) \neq \varnothing$. Then:

(1) $\mathcal{P}_{J}(H)$ has a unique member $\Gamma_{J}(H)$.

(2) $\Gamma_{J}(H)$ is the greatest member of $\mathcal{P}^{\prime}(H)$.

(3) Each $M \in \mathcal{O}_{G}(H)^{\prime}$ is transitive and imprimitive on $\Omega, \mathcal{P}^{\prime}(M)$ has a greatest member $\Gamma_{M}, \Gamma_{M} \leq \Gamma_{J}$, and $D\left(\Gamma_{J}\right) \leq \hat{G}_{\Gamma_{M}}$.

(4) $\Gamma_{J}(H) \in \mathcal{P}_{2}(H)$.

Proof. Let $M \in \mathcal{O}_{G}(H)^{\prime}, \Gamma_{0} \in \mathcal{P}_{J}(H)$, and $D_{0}:=D\left(\Gamma_{0}\right)$. As $H_{*} \not \leq J$, we get $H_{*} \not \leq D\left(\Gamma_{0}\right)$, so $\Gamma_{0} \in \mathcal{P}_{2}(H)$ by 6.3.1. By definition, $D_{0} \leq J \leq H \leq M$, so by 6.4.3, (3) holds with $\Gamma_{J}$ replaced by $\Gamma_{0}$. Then specializing to the case $\Gamma \in \mathcal{P}^{\prime}(H)$ and $M=N_{G}(\Gamma)$, we conclude $\Gamma_{0}$ is the greatest member of $\mathcal{P}^{\prime}(H)$. As this holds for each $\Gamma_{0} \in \mathcal{P}_{J}$, part (1) follows with $\Gamma_{0}=\Gamma_{J}$, and then (2)-(4) follow from earlier remarks which showed these statements hold for $\Gamma_{0}$.

Notation 6.7. Suppose $\mathcal{P}_{J}(H) \neq \varnothing$. In that event set

$$
\Gamma_{J}:=\Gamma_{J}(H), \quad M_{J}:=M\left(\Gamma_{J}\right), \quad \text { and } \quad M_{J}^{*}:=M_{J} / G_{\Gamma_{J}} .
$$

For $\Sigma \in \mathcal{P}^{\prime}(H)$, we define $\Sigma^{*}$ to be the partition of $\Gamma_{J}$ with blocks $\sigma^{*}=\left(\Gamma_{J}\right)_{\sigma}$ for $\sigma \in \Sigma$. By 6.6.2, $\Sigma \leq \Gamma_{J}$, so this makes sense. Define $\mathcal{P}^{\prime}\left(H^{*}\right)$ to be the set of nontrivial $H^{*}$-invariant partitions of $\Gamma_{J}$. In the other direction, for $\Xi \in \mathcal{P}^{\prime}\left(H^{*}\right)$, 
define $\varphi(\Xi) \in \mathcal{P}^{\prime}(H)$ to be the partition with blocks

$$
\phi(\xi)=\bigcup_{\alpha \in \xi} \alpha \text { for } \xi \in \Xi .
$$

For $W \in \mathcal{W}_{0}$, define $\Gamma_{W}:=\Gamma_{W H}$ using 6.6.3, and set

$$
M_{W}:=M\left(\Gamma_{W}\right) \quad \text { and } \quad D_{W}:=D\left(\Gamma_{W}\right) .
$$

6.8. Assume $\mathcal{P}_{J}(H) \neq \varnothing$, and let $\mathcal{P}_{*}(H)$ denote the set of maximal members of $\mathcal{P}_{2}(H)-\left\{\Gamma_{J}\right\}$. Then:

(1) The map $\varphi: \mathcal{P}^{\prime}\left(H^{*}\right) \rightarrow \mathcal{P}^{\prime}(H)-\left\{\Gamma_{J}\right\}$ is an isomorphism of posets with inverse $\Sigma \mapsto \Sigma^{*}$.

(2) For $W \in \mathcal{W}_{0}, \Gamma_{W} \leq \Gamma_{J}, D_{W} \leq W$, and $\Gamma_{W} \in \mathcal{P}_{2}(H)$.

(3) $\mathcal{P}_{*}(H)$ has a unique member $\Gamma_{*}=\Gamma_{*}(H)$.

(4) $\Gamma_{*}^{*}$ is the greatest member of $\mathcal{P}^{\prime}\left(H^{*}\right)$.

(5) $U:=\bar{D}\left(\Gamma_{*}\right) \in W_{0}^{*}$ and $N_{U}\left(\Gamma_{J}\right)=J$.

(6) For $\gamma \in \Gamma_{*}$, define $\tilde{J}_{\gamma}:=J \cap A_{\Omega-\gamma}$. Then $\tilde{J}_{\gamma}=N_{A_{\Omega-\gamma}}\left(\gamma^{*}\right)$.

(7) $J_{\Gamma_{J}-\gamma}^{*}=\operatorname{Sym}\left(\Gamma_{J}\right)_{\Gamma_{J}-\gamma^{*}}$.

(8) Each member of $\mathcal{O}_{M_{J}^{*}}\left(H^{*}\right)^{\prime}$ is imprimitive on $\Gamma_{J}$.

(9) $\Gamma_{*}$ is the unique $\Sigma \in \mathcal{P}^{\prime}(H)$ such that $\bar{D}(\Sigma) \in W_{0}^{*}$.

Proof. Part (1) follows from 6.6.2. By 6.6.4, $\Gamma_{J} \in \mathcal{P}_{2}(H)$. Further $D_{J} \leq J \leq H$, so for $W \in W_{0}, D_{J} H \leq W H$. Thus (2) follows from parts (3) and (4) of 6.4 applied to $\Gamma_{J}$ in the role of " $\Gamma$ ".

Let $\mathcal{W}_{1}:=\left\{W \in \mathcal{W}_{0}: \Gamma_{W} \neq \Gamma_{J}\right\}$. By 3.3, $W_{1} \neq \varnothing$. Pick $U \in \mathcal{W}_{1}$. By (2), $D_{U} \leq U, \Gamma_{U} \in \mathcal{P}_{2}(H)$, and $\Gamma_{U} \leq \Gamma_{J}$, so as $\Gamma_{U} \neq \Gamma_{J}, \Gamma_{U}<\Gamma_{J}$. If $D_{U} \leq J$, then $\Gamma_{U}=\Gamma_{J}$ by 6.6.1, which we just saw is not the case. Therefore $D_{U} \not J J$. Also we have $\mathcal{P}_{*}(H) \neq \varnothing$, so taking $U:=\bar{D}(\Gamma)$ for $\Gamma \in \mathcal{P}_{*}(H)$, we may assume $\Gamma_{U}=\Gamma \in \mathcal{P}_{*}(H)$, and we have shown $U \not \leq J$.

Suppose $V \in \mathcal{W}_{0}^{*}$ and $V<U$. Then by definition in $6.7, \Gamma_{V}$ is the unique maximal member of $\mathcal{P}^{\prime}(H V)$, so as $H V \leq H U$, we conclude that $\Gamma \leq \Gamma_{V}$. Hence as $\Gamma_{*} \in \mathcal{P}_{*}(H)$, we get $\Gamma_{V}=\Gamma$ or $\Gamma_{J}$ by maximality of $\Gamma$. In the former case, $D(\Gamma)=D_{V} \leq V$ by (2), so $U=\bar{D}(\Gamma) \leq V$, contradicting $V<U$. Thus we have $V \leq N_{U}\left(\Gamma_{J}\right)$, so $N_{U}\left(\Gamma_{J}\right)$ is the unique maximal member of $\left\{W \in \mathcal{W}_{0}: W<U\right\}$, contrary to 3.4 .

Thus no such $V$ exists, so $U \in W_{0}^{*}$. Set $E:=N_{U}\left(\Gamma_{J}\right)$. Now $E$ is $H$-invariant, so $\bar{E} \in \mathcal{W}_{0}$ by 2.7. Then as $U \in \mathcal{W}_{0}^{*}, \bar{E}=U$ or $J$. As $\Gamma_{J} \neq \Gamma, \bar{E} \neq U$, so $E \leq J$. 
However $D(\Gamma)=D_{U} \leq U$ and $N_{D(\Gamma)}\left(\Gamma_{J}\right)$ is a maximal $H$-invariant subgroup of $D(\Gamma)$ by 3.7.2 in [8], using the fact that $D\left(\Gamma_{J}\right) \leq H$ when the blocks of $\Gamma_{J}$ are of size 2 , and those of $\Gamma$ are of size 8 . Therefore as $U=\bar{D}(\Gamma) \in W_{0}^{*}$, it follows that $D(\Gamma) \cap J=N_{D(\Gamma)}\left(\Gamma_{J}\right)$ and $J=N_{U}\left(\Gamma_{J}\right)$. This establishes (5), once we prove (3). Further as $J=N_{U}\left(\Gamma_{J}\right)$ and $D(\Gamma) \leq U$, (6) follows.

Next let $\alpha, \beta \in \Gamma_{J}$ with $\alpha, \beta \subseteq \gamma \in \Gamma$, and set $m:=|\alpha|$. We next prove (7). By (6), it suffices to show there is a transposition $t \in M_{J}^{*}$ with cycle $(\alpha, \beta)$ on $\Gamma_{J}$, such that $t=s^{*}$ for some $s \in A_{\Omega-(\alpha \cup \beta)}$. If $m$ is even, we can choose $s$ so that $s^{*}$ is of cycle type $2^{m}$. Thus we may assume $m$ is odd. Now $m-1=2^{a} k$ with $k$ odd and $a>0$. For $\delta \in\{\alpha, \beta\}$, let $t_{\delta} \in \operatorname{Sym}\left(\Gamma_{J}\right)$ have one fixed point and $k$ cycles of length $2^{a}$, with $\operatorname{Mov}\left(t_{\delta}\right) \subseteq \delta$. Let $t \in \operatorname{Sym}\left(\Gamma_{J}\right)$ interchange $\alpha$ and $\beta$ with $t^{2}=t_{\alpha} t_{\beta}$ and $\operatorname{Mov}(t)=\alpha \cup \beta$. Then $t$ has one cycle of length 2 and $k$ cycles of length $2^{a+1}$, so $t$ is even and hence $t \in M_{J}^{*}$. Thus (7) holds.

Now (7) and 3.2.1 in [8] applied to $M_{J}^{*}$ acting on $\Gamma_{J}$ imply (8), and, together with 3.2.2 in [8], say that $\Gamma^{*}$ is the greatest member of $\mathcal{P}^{\prime}\left(H^{*}\right)$. As $\Gamma$ can be chosen to be any member of $\mathcal{P}_{*}(H)$, (3) and (4) follow from this fact and (1).

Let $\delta$ consist of those $\Sigma \in \mathcal{P}^{\prime}(H)$ such that $\bar{D}(\Sigma) \in \mathcal{W}_{0}^{*}$. By (5), $\Gamma_{*} \in \mathcal{S}$. Conversely suppose $\Sigma \in \mathcal{S}$ and set $V:=\bar{D}(\Sigma)$. Then $\Sigma \in \mathcal{P}_{2}(H)$ and $\Sigma \leq \Gamma_{*}$ by (1) and (4). As $\Gamma_{*}<\Gamma_{J},\left|\Gamma_{*}\right|<n / 2$, so $D\left(\Gamma_{*}\right) \leq D(\Sigma)$ by 6.4.2. But now $U=\bar{D}\left(\Gamma_{*}\right) \leq \bar{D}(\Sigma)=V$, so as $V \in \mathcal{W}_{0}^{*}, U=V$, and hence $\Sigma=\Gamma_{*}$. Thus (9) is established.

6.9. $\mathcal{P}_{J}(H)=\varnothing$.

Proof. Assume otherwise and adopt Notation 6.7 together with the notation in 6.8. Set $U:=\bar{D}\left(\Gamma_{*}\right)$. Thus $U \in \mathcal{W}_{0}^{*}$ by 6.8 .5 . Let $\mathcal{C}$ be the connected component of $\Lambda$ containing $U$ and $Y:=\hat{G}_{\Gamma_{J}}$. As $\Lambda$ is disconnected there exists $V \in W_{0}^{*}$ with $V \notin \mathcal{C}$. By 6.8.9, $V \neq \bar{D}_{V}$, while $\bar{D}_{V} \in \mathcal{W}_{0}$ is contained in $V$ by 6.8.2. Thus we have $D_{V} \leq J$, so by 6.6.1, $\Gamma_{V}=\Gamma_{J}$, so $H V \leq N_{G}\left(\Gamma_{J}\right)=M_{J}$. Next by 6.8.7,

$$
\left(M_{J}^{*}\right)_{\Gamma_{*}^{*}} \leq J^{*} \leq V^{*},
$$

so by 6.8.7 and 3.2 in [8], either $\operatorname{Alt}\left(\Gamma_{J}\right) \leq V^{*}$ or $\mathcal{P}^{\prime}\left(H^{*} V^{*}\right)$ has a greatest member $\Sigma^{*}, \Sigma^{*} \leq \Gamma_{*}^{*}$, and $\left(M_{J}^{*}\right)_{\Sigma^{*}} \leq V^{*}$. As $Y \leq J$ and $H_{*} \not \leq V$, the latter holds.

Next $H^{*} \cap\left(M_{J}^{*}\right)_{\Sigma^{*}} \leq H^{*} \cap V^{*}=(H \cap V)^{*}=J^{*}$ as $Y \leq J$. Thus the kernel of the action of $H_{*}$ on $\Sigma^{*}$ is contained in $J$, so from 6.8.1, the kernel of the action of $H_{*}$ on $\Sigma:=\varphi\left(\Sigma^{*}\right)$ is also contained in $J$, and hence $\Sigma \in \mathcal{P}_{2}$ by 6.3.1, so we obtain $E:=\bar{D}(\Sigma) \in \mathcal{W}_{0}$ by 6.4.1. By 6.8.4, $\Sigma \leq \Gamma_{*}$. As we saw during the proof of 6.8.9, $\left|\Gamma_{*}\right|>n / 2$, so by 6.4.2, $D\left(\Gamma_{*}\right) \leq D(\Sigma)$, and hence $U=\bar{D}\left(\Gamma_{*}\right) \leq E$. Moreover $D(\Sigma) \cap M_{J}=: E_{J}$ is $H$-invariant, so $\bar{E}_{J} \in \mathcal{W}_{0}$. Also

$$
E_{J}^{*} \leq\left(M_{J}^{*}\right)_{\Sigma^{*}} \leq V^{*},
$$


so $\bar{E}_{J} \leq V$. If $\Sigma \neq \Gamma_{*}$, then $E_{J}$ does not act on $\left(M_{J}^{*}\right)_{\Gamma_{*}^{*}} \unlhd J^{*}$, so $E_{J} \not \leq J$. But then $U, E, \bar{E}_{J}, V$ is a path in $\Lambda^{\prime}$, contradicting $V \notin \mathcal{C}$. Hence $\Sigma=\Gamma_{*}$, so $V$ acts on $D\left(\Gamma_{*}\right)$. Then by 3.9.3 in [6], $U V=H_{*} V \leq M_{J}$, which is impossible as $D_{U}$ does not act on $D_{J}$. This completes the proof of the lemma.

\section{$7 \quad \mathcal{P}_{2}(H) \neq \varnothing$}

In this section we assume:

Hypothesis 7.1. Hypothesis 6.1 holds and $\mathcal{P}_{2}(H) \neq \varnothing$.

Notation 7.2. For $\Sigma \in \mathcal{P}_{2}(H)$, write $\Sigma=\left\{\sigma_{1}, \ldots, \sigma_{m}\right\}$, where $m=|\Sigma|$, and set $k(\Sigma)=\left|\sigma_{i}\right|$, the size of the blocks in $\Sigma$. If $k(\Sigma) \neq 2$, by definition of $D=D(\Sigma)$, $D=D_{1} \times \cdots \times D_{m}$, where $D_{i}=A_{\Omega-\sigma_{i}}$ acts faithfully as the alternating group $A_{k(\Sigma)}$ on $\sigma_{i}$.

7.3. Let $\Sigma \in \mathcal{P}_{2}(H)$. Then:

(1) $\bar{D}(\Sigma) \in \mathcal{W}_{0}^{\prime}$.

(2) $|\Sigma| \geq 5$.

Proof. By 6.4.1, $U:=\bar{D}(\Sigma) \in \mathcal{W}_{0}$. By 6.9, we have $\Sigma \notin \mathcal{P}_{J}(H)$, so $D(\Sigma) \not \leq J$, and hence $U \in W_{0}^{\prime}$. That is (1) holds.

As $\Sigma \in \mathcal{P}_{2}(H), H_{*} \not \leq G_{\Sigma}$, so $H_{*}^{\Sigma} \neq 1$. Then as $H_{*}=H_{*}^{\infty},(2)$ holds.

7.4. Suppose $\Sigma \in \mathcal{P}_{2}(H)$ with $k(\Sigma) \neq 2$. Let $D:=D(\Sigma)$ and $K:=\hat{G}_{\Sigma}$. Then:

(1) $\bar{K} \in W_{0}^{\prime}$.

(2) $K=D X$, where $\bar{X} \in \mathcal{W}_{0}$ with $\bar{D} \cap \bar{X}=J$ and $\bar{X} \cap K=X$.

(3) $\mathcal{W}_{0}(\leq \bar{D})=\left\{\bar{B}: B \in \mathcal{V}_{D}(H)\right\}$ and $\mathcal{W}_{0}(\leq \bar{K})=\left\{\bar{B}: B \in \mathcal{V}_{K}(H)\right\}$.

(4) Each member of $\mathcal{V}_{D}(H)$ is $X H$-invariant.

(5) In the notation of $2.5, \Xi_{K H} \cong \mathcal{V}_{K}(H) \cong \Delta(r), \Xi_{D H} \cong \mathcal{V}_{D}(H) \cong \Delta(s)$, and $\Xi_{X H} \cong \mathcal{V}_{X}(H) \cong \Delta(r-s)$ for some $1 \leq s \leq r$.

Proof. By 6.4.1, $\bar{K} \in W_{0}$, so (1) follows from 7.3.1.

We next prove (2), by applying 3.7 to $D, K H$ in the roles of $Y, X$, and with $\mathcal{C}$ the connected component of $\bar{D}$. Then, in the notation of 3.7, we have $\bar{K}=L_{\mathscr{D}}$ and $\bar{D}=L_{\mathscr{A}}$. Set $V:=L_{\mathscr{B}}$ and $X:=V \cap K$. Then by construction,

$$
V \cap \bar{D}=L_{\mathscr{B}} \cap L_{\mathscr{A}}=L_{\mathscr{B} \cap \mathcal{A}}=L_{\varnothing}=J,
$$

and as $D \unlhd \bar{K}$,

$$
\bar{K}=\mathscr{L}_{\mathscr{D}}=\left\langle\mathscr{L}_{\mathscr{A}}, \mathscr{L}_{\mathscr{B}}\right\rangle=D V,
$$


so $K=K \cap D V=D(K \cap V)=D X$. Finally $\bar{K}=K J=D X J=D \bar{X}$ so

$$
V=V \cap \bar{K}=V \cap D \bar{X}=(V \cap D) \bar{X}=J \bar{X}=\bar{X},
$$

completing the proof of (2).

Moreover as $\bar{K} \in \mathcal{W}_{0}^{\prime}$, (3) follows from 3.7.3. Then 3.7.4 and (3) imply (4). Next 2.5 says

$$
\Xi_{K H} \cong \mathcal{W}_{0}(\leq \bar{K}) \cong \Delta(r)
$$

for some $r \geq 1$, and indeed $\mathscr{D}=W_{0}^{*} \cap \bar{K}$, so $r=|\mathscr{D}|$. Similarly

$$
\Xi_{K H} \cong \mathcal{W}_{0}(\leq \bar{D}) \cong \Delta(s)
$$

with $\mathcal{A}=\mathcal{W}_{0}^{*} \cap \bar{D}$ and $s=|\mathcal{A}|$, so $\Xi_{X K} \cong \Delta(t)$ with $t=|\mathscr{B}|=r-s$, establishing (5).

7.5. Assume $\Sigma \in \mathcal{P}_{2}(H)$ with $k(\Sigma) \geq 5$. Define $K:=\hat{G}_{\Sigma}$ and $X$ as in 7.4. Then for each $V \in \mathcal{V}=\mathcal{W}_{0}(\leq \bar{D})$ and each $1 \leq i \leq m:=|\Sigma|$,

(1) $V \cap D=\prod_{i} V_{i}$, where $1 \neq V_{i}=V \cap D_{i}$.

(2) $V \cap D$ is $X H$-invariant.

(3) $N_{H}\left(\sigma_{i}\right)^{\sigma_{i}}$ is primitive.

(4) $\Sigma$ is maximal in $\mathcal{P}^{\prime}(H)$.

(5) $J_{i}$ is transitive on $\sigma_{i}$.

Proof. Adopt the notation of 7.4 and its proof. Then $\bar{D}=L_{\mathcal{A}}, \bar{X}=L_{\mathcal{B}}$, and by 3.7.4, for each $\alpha \subseteq \mathcal{A}, D_{\alpha}=D \cap L_{\alpha}$ is $X H$-invariant. Pick $\alpha$ to be maximal proper subset of $\mathcal{A}$, and set $M:=H D_{\alpha}$ and $Y:=H D$. Then by 7.4.3, $M$ is maximal in $Y$. Further $\bar{D}_{\alpha}=V \in \mathcal{V}$.

Let $B:=H K, F:=H X, B^{i}:=N_{B}\left(\sigma_{i}\right)^{\sigma_{i}}$, and for $P \leq B, P^{i}:=N_{P}\left(\sigma_{i}\right)^{\sigma_{i}}$. Then $B^{i}=\operatorname{Sym}\left(\sigma_{i}\right), D^{i}=D_{i}^{i}=\operatorname{Alt}\left(\sigma_{i}\right)$, and $X^{i} \not \leq \operatorname{Alt}\left(\sigma_{i}\right)$, so $F^{i} \not \leq \operatorname{Alt}\left(\sigma_{i}\right)$.

As $k=k(\Sigma) \geq 5, D_{i} \cong A_{k}$ is a nonabelian simple group from 7.2. Thus we have $F^{*}(Y)=D$ and $H \leq M$, so $M$ is transitive on the set $\left\{D_{1}, \ldots, D_{m}\right\}$ of components of $Y$. Thus, in the language of [4], $Y$ is faithful and primitive on $Y / M$, and is complemented, diagonal, or semisimple. In the last case,

$$
M \cap D=M_{1} \times \cdots \times M_{m},
$$

where $M_{i}=M \cap D_{i} \neq 1$. Then as

$$
M \cap D=H D_{\alpha} \cap D=(H \cap D) D_{\alpha}=(J \cap D) D_{\alpha}=D_{\alpha},
$$

we have $V=\prod_{i} V_{i}$. In particular if $Y$ on $Y / H D_{\alpha}$ is semisimple for each maximal $\alpha$, then (1) holds, modulo the assertion that $W_{i} \neq 1$ for each $W \in \mathcal{V}$, which we now prove. 
Suppose $W_{i}=1$. Then $W \cap D=1$, so $W=J$. Now $H^{i} \cap D^{i}$ is $H^{i}$-invariant, so $H^{i} \cap D^{i}=U_{i}^{i}$ for some $U=L_{\gamma}$ and $\gamma \subset \mathcal{A}$ by 7.4.3. As $H^{i}$ acts on $Z_{i}$ for each $Z \in \mathcal{V}$, also $U_{i}$ acts on $Z_{i}$. Claim $\gamma=\varnothing$, so that $U=J$ and hence $U_{i}^{i}=$ $J_{i}^{i}=1$. For if not, $\delta=\mathcal{A}-\gamma \neq \varnothing$ or $\mathcal{A}$, so by 3.7.3, $Z:=L_{\delta} \neq J$ or $D$, and $U_{i}$ acts on $Z_{i}$. Then by 3.7.3, $D=\left\langle U_{i}, Z_{i}\right\rangle$ acts on $Z_{i} \neq 1$ or $D$, a contradiction. This establishes the claim, and hence $H^{i} \cap D^{i}=1$. Therefore $\left|H^{i}\right| \leq 2$. But then as $\mathcal{O}_{D_{i}}\left(N_{H}\left(\sigma_{i}\right)\right)=\left\{W_{i}: W \in \mathcal{A}\right\} \cong \Delta(s)$ by 7.4.5, we have a contradiction.

Thus we may assume $Y$ is complemented or diagonal. But by 7.4.2, $K=D X$ and $D_{\alpha}$ and $D \cap J$ are $H X$-invariant. Now by 7.4.2, $X \cap D \leq J$, while

$$
\operatorname{Aut}_{X}\left(D_{i}\right) \not \leq \operatorname{Inn}\left(D_{i}\right)
$$

by paragraph two of the proof. Thus if $Y$ is complemented, then $D \cap J=D_{\alpha}=1$, so

$$
\operatorname{Aut}_{X}\left(D_{i}\right) \cong Z_{2} \text { and } \operatorname{Aut}_{M}\left(D_{i}\right)=\operatorname{Aut}_{H}\left(D_{i}\right) .
$$

Then as $\operatorname{Aut}_{H}\left(D_{i}\right)$ acts on $\operatorname{Aut}_{X}\left(D_{i}\right), \operatorname{Inn}\left(D_{i}\right) \not \leq \operatorname{Aut}_{M}\left(D_{i}\right)$, contradicting $Y$ complemented.

This leaves the case $Y$ diagonal. In this case $D_{\alpha}=\prod_{\rho} D_{\alpha, \rho}$, where $D_{\alpha, \rho}$ is a full diagonal subgroup of $D_{\rho}, \rho$ varies over the blocks of an $H$-invariant partition of $\{1, \ldots, m\}$, and $D_{\rho}=\prod_{i \in \rho} D_{i}$. As $K=D X, D_{\alpha}$ is $X$-invariant, and $m>2$ by 7.3.2, this too is a contradiction. So (1) is established.

Part (2) follows from 7.4.4.

Suppose $H^{i}$ is not primitive. Then $H^{i} \leq P^{i}$ the stabilizer of some nontrivial partition $\Gamma$ of $\sigma_{i}$. Let $P_{i}:=N_{D_{i}}(\Gamma)$ and $P:=\left\langle P_{i}^{H}\right\rangle$. Then $P \in \mathcal{V}_{D}(H)$, so by 7.4.4, $X$ acts on $P$. Therefore $F^{i}$ acts on $P_{i}$, and hence $F^{i}$ is also imprimitive. Therefore by 7.4 .5 and 5.2 in [8], $\mathcal{O}_{B^{i}}\left(F^{i}\right)$ is the lattice with maximal members $\left\{B_{1}^{i}, \ldots, B_{s}^{i}\right\}$, where $B_{j}^{i}$ is the stabilizer of a nontrivial partition $\Gamma_{j}$ of $\sigma_{i}$, and $\Gamma_{1}<\cdots<\Gamma_{s}$. Then setting $P_{i, j}=N_{D_{i}}\left(\Gamma_{j}\right)$ and $P_{j}=\left\langle P_{i, j}^{H}\right\rangle$, it follows from 7.4 that the maximal members of $W_{0}(<\bar{D})$ are $\bar{P}_{j}, 1 \leq j \stackrel{<}{\leq} s$. Let $\Sigma_{j}$ be the union of the images of the blocks in $\Gamma_{j}$ under $H$. Then $\Sigma_{j} \in \mathcal{P}^{\prime}(H)$ with $\Sigma<\Sigma_{j}$. Further

$$
J=\bigcap_{j} \bar{P}_{j}
$$

contains $D\left(\Sigma_{s}\right)$. But then we get $\Sigma_{s} \in \mathcal{P}_{J}(H)$, contrary to 6.9. This completes the proof of (3), and (3) implies (4). Finally as $J_{i}$ is $N_{H}\left(\sigma_{i}\right)$-invariant, (1) and (3) imply (5).

7.6. Assume $\Sigma \in \mathcal{P}_{2}(H)$ and $k(\Sigma)=4$. Let $D:=D(\Sigma)$ and $U:=\bar{D}$. Then:

(1) $O_{2}(D)=D \cap J$ and $U \in W_{0}^{*}$.

(2) For each $W \in W_{0}, W H$ is imprimitive. 
Proof. Let $Q:=O_{2}(D), \sigma:=\sigma_{1}$, and assume $Q \not \leq J$. By 7.3.1, $U \in \mathcal{W}_{0}^{\prime}$, so $\bar{Q} \in W_{0}^{\prime}$ by 2.7.1. Further either $D=Q(J \cap D)$ or $\bar{Q}<U$. In any event by 3.7, there exists $Y \leq D$ such that $D=Q Y, \bar{Y} \in \mathcal{W}_{0}$, and $\bar{Q} \cap \bar{Y}=J$. Thus

$$
Y \cap Q=Q \cap J .
$$

Let $M:=Y H$. Then $M$ is transitive on $\Sigma$ and $Y$ is irreducible on $Q_{i}:=Q \cap D_{i}$, so $M$ is irreducible on $Q$. Thus as $Q \not \leq J$ and $Q \cap J=Y \cap Q \unlhd M$, we conclude that $Q \cap J=1$. Thus as $Q \cap J=Y \cap Q$ and $D=Q Y, Y$ is a complement to $Q$ in $D$. That is $Y \in \operatorname{Syl}_{3}(D)$. Therefore

$$
Y_{1}:=Y \cap D_{1} \in \operatorname{Syl}_{3}\left(D_{1}\right) .
$$

But $Y=\bar{Y} \cap D$ and $\bar{Y}$ is $H$-invariant, so $N_{H}(\sigma)$ acts on $Y_{1}$. This is impossible as $Y_{1}$ fixes a unique point of $\sigma$, and $H$ is transitive on $\Omega$, so $N_{H}(\sigma)$ is transitive on $\sigma$.

Therefore $Q \leq J$. Now define $K$ and $X$ as in 7.4, and let $M:=X H$. Then $K=D X$ and by 7.4.2,

$$
X \cap D=J \cap D,
$$

so $Q \leq X$. Set $D^{*}=D / Q$. Then $D_{1}^{*}=\left[D_{1}^{*}, X^{*}\right]$ and $H$ is transitive on $\Sigma$, so $M$ is irreducible on $D^{*}$. Thus either $Q=X \cap D$ or $D \leq X$, and the latter is impossible as $X \cap D=J \cap D$ and $D \not \leq J$ by 7.3.1. Hence $Q=\bar{X} \cap D$, so as $Q \leq J \leq \bar{X}$, $Q=J \cap D$. Further as $X$ is irreducible on $D^{*}$ and $Q \leq J, U \in \mathcal{W}_{0}^{*}$. This completes the proof of (1).

Next $E:=Q_{\Omega-\sigma} \cong E_{4}$ and for each $e \in E^{\#}, \operatorname{Mov}(e)=\sigma$. Let $W \in W_{0}$. Then $E \leq J \leq W$, and by 2.14, $W H$ is not almost simple. Therefore by 4.2 in [8], $W H$ is imprimitive, establishing (2).

7.7. For $\Sigma \in \mathcal{P}_{2}(H), k(\Sigma) \neq 3$.

Proof. Assume $\Sigma \in \mathcal{P}_{2}(H)$ with $k(\Sigma)=3$, let $D:=D(\Sigma), U:=\bar{D}$, and adopt the notation of 7.4. Set $M:=H X$ and $Y:=H K$. Then $N_{M}\left(D_{1}\right)$ is irreducible on $D_{1}$ and $H$ is transitive on $\Sigma$, so $M$ is irreducible on $D$. But, using 7.4.2,

$$
M \cap K=H X \cap K=X(H \cap K)=X(J \cap K)=X,
$$

so $M \cap D=X \cap D$. Thus as $M$ is irreducible on $D, X \cap D=1$ or $D$. By 7.4.2, $X \cap D=J \cap D$, so as $D \not \leq J$ by 7.3.1, $D \cap X=1$. By construction in 7.4.2, $K=D X$, so $X$ is a complement to $D$ in $K$. That is $X \in \operatorname{Syl}_{2}(K)$. Let $\sigma=\sigma_{1}$. Now $K^{\sigma}=\operatorname{Sym}(\sigma) \cong S_{3}$ and $H$ acts on $X$, so $H^{\sigma}$ acts on $X^{\sigma} \in \operatorname{Syl}_{2}\left(D^{\sigma}\right)$. This is impossible as $X^{\sigma}$ fixes a unique point of $\sigma$, whereas $H$ is transitive on $\Omega$, so $H^{\sigma}$ is transitive on $\sigma$. 
7.8. Assume $\Sigma \in \mathcal{P}_{2}(H)$ and $k(\Sigma)=2$. Let $D:=D(\Sigma), J_{D}:=J \cap D, U:=\bar{D}$, and $\mathcal{W}_{0}^{*} \cap U=\left\{U_{1}, \ldots, U_{r}\right\}$. Then $J_{D} \unlhd H D$, and setting $D^{*}=D / J_{D}$,

$$
D^{*}=X_{1}^{*} \oplus \cdots \oplus X_{r}^{*},
$$

where $X_{i}:=U_{i} \cap D, X_{i}^{*}=\left[X_{i}^{*}, H\right]$, and for $i \neq j, X_{i}^{*}$ and $X_{j}^{*}$ are inequivalent $\boldsymbol{F}_{2} H$-submodules of $D^{*}$.

Proof. As $H$ acts on the abelian group $D$ and $J \unlhd H, J_{D} \unlhd H D$.

By 7.3.1, $U \in W_{0}^{\prime}$, so by 3.7.3,

$$
D=U \cap D=\left\langle X_{i}: 1 \leq i \leq r\right\rangle,
$$

and $\mathcal{X}=\left\{X_{i}^{*}: 1 \leq i \leq r\right\}$ is the set of irreducible $\boldsymbol{F}_{2} H$-submodules of $D^{*}$. By 3.5.2, $X_{i}^{*}=\left[X_{i}^{*}, H\right]$. If $i \neq j$, then by 3.7.3, $X \cap\left(X_{i}^{*}+X_{j}^{*}\right)=\left\{X_{i}^{*}, X_{j}^{*}\right\}$, so $X_{i}^{*}$ is not $\boldsymbol{F}_{2} H$-isomorphic to $X_{j}^{*}$, completing the proof.

7.9. Assume there exists $\Sigma \in \mathcal{P}_{2}(H)$ such that $k(\Sigma) \geq 3$. Then for each $W \in W_{0}$, $H W$ is imprimitive on $\Omega$.

Proof. Let $1 \neq W \in \mathcal{W}_{0}$ and $Y:=H W$. By 2.14 :

(*) $Y$ is not almost simple.

Choose $\Sigma \in \mathcal{P}_{2}(H)$ and set $D:=D(\Sigma), U:=\bar{D}, k:=k(\Sigma)$. We may assume $k>2$ and $Y$ is primitive, and it remains to derive a contradiction. By 7.6.2 and 7.7, $k>4$. Set $\sigma:=\sigma_{1}$ and $J_{1}:=J \cap D_{1}$. As $k>4, J_{1}$ is transitive on $\sigma$ by 7.5.5.

Next $J_{1} \leq Y$ and $Y$ is primitive on $\Omega$, so by 3.5 in [8] and (*), $n=2^{a+1}$ for some $a>1, Y$ is the stabilizer of an affine structure, and $J_{1} \not \leq E:=F^{*}(Y)$. As $Y$ is the stabilizer of an affine structure, $E$ is the unique minimal normal subgroup of $Y$, so $E \leq W$. Further $J_{1} \leq J \leq W$, so as $J_{1} \not \leq E, E<W$. However as $Y$ is the stabilizer of an affine structure over $\boldsymbol{F}_{2}$ and $n>4, Y / E$ is simple, so $W=Y$. This is a contradiction as $F^{*}(Y / W) \cong L \neq 1$.

7.10. For each $\Sigma \in \mathcal{P}_{2}(H), \bar{D}(\Sigma) \in W_{0}^{!}$.

Proof. Let $\Sigma \in \mathcal{P}_{2}(H), D:=D(\Sigma)$, and $U:=\bar{D}$. Assume $U \notin W_{0}^{!}$; we derive a contradiction.

Let $k:=k(\Sigma)$, let $\left\{W_{i}: 1 \leq i \leq t\right\}$ be the members of $W_{0}$ such that $U$ is a maximal $H$-invariant subgroup of $W_{i}$, and set $Y_{i}:=W_{i} H$. Then $D \leq W_{i} \leq Y_{i}$, so by parts (3) and (4) of 6.4:

(1) For $1 \leq i \leq t, Y_{i}$ is imprimitive on $\Omega, \mathcal{P}^{\prime}\left(Y_{i}\right)$ has a greatest member $\Gamma_{i}$, $\Gamma_{i} \in \mathcal{P}_{2}$, and $\Gamma_{i} \leq \Sigma$. 
Next by 3.8:

(2) For some $1 \leq j \leq t, W_{j}$ does not act on $D$. Thus $\Gamma_{j} \neq \Sigma$, so $\Gamma_{j}<\Sigma$.

Let $\Gamma:=\Gamma_{j}$ and $k^{\prime}:=k(\Gamma)$. If $k^{\prime}>4$, then $\Gamma$ is maximal in $\mathcal{P}^{\prime}(H)$ by 7.5.4, contradicting $\Gamma<\Sigma$. Similarly if $k^{\prime}=2$, then $\Gamma$ is maximal in $\mathscr{P}^{\prime}(H)$, so it follows from 7.7 that:

(3) $k^{\prime}=4$.

However as $k^{\prime}=4,7.6 .1$ says that $W_{j} \in W_{0}^{*}$, contradicting $J<U<W_{j}$. This completes the proof of the lemma.

7.11. For $\Sigma \in \mathcal{P}_{2}(H), k(\Sigma) \neq 4$.

Proof. Assume $\Sigma \in \mathcal{P}_{2}(H)$ with $k(\Sigma)=4$. Then by 7.6.1, $\bar{D}(\Sigma) \in \mathcal{W}_{0}^{*}$, contrary to 7.10 .

7.12. Assume $\Sigma \in \mathcal{P}_{2}(H)$ with $k(\Sigma)>2$. Then:

(1) $k(\Sigma)>4$.

(2) $\mathcal{P}_{2}(H)=\{\Sigma\}$.

(3) For each $\Gamma \in \mathcal{P}^{\prime}(H)$, $\Gamma \leq \Sigma$.

Proof. By 7.7 and 7.11, $k:=k(\Sigma)>4$. Adopt the notation of 7.5 and set $\sigma:=\sigma_{1}$. By 7.5.5, $J_{1}^{\sigma}$ is transitive. Let $\Gamma \in \mathcal{P}^{\prime}(H)$ and $\gamma \in \Gamma$ with $\sigma \cap \gamma \neq \varnothing$. As $J_{1}^{\sigma}$ is transitive, 1.1 says that $\sigma \subseteq \gamma$ or $\gamma \subseteq \sigma$. Thus as $H$ is transitive on $\Gamma$ and $\Sigma$, it follows that $\Gamma$ and $\Sigma$ are comparable. Further if $\Sigma \leq \Gamma$, then $\Sigma=\Gamma$ by 7.5.4. This establishes (3).

Suppose $\Gamma \in \mathcal{P}_{2}(H)-\{\Sigma\}$. Then by (3), $\Gamma<\Sigma$. By 6.4.2, $D<D(\Gamma)$, so by 7.10, $\bar{D}=\bar{D}(\Gamma)$. But then $D(\Gamma)=D J \leq N_{G}(D)$, a contradiction. Hence (2) holds.

7.13. For each $\Sigma \in \mathcal{P}_{2}(H), k(\Sigma)=2$.

Proof. Assume $\Sigma \in \mathcal{P}_{2}(H)$ with $k:=k(\Sigma) \neq 2$. By 7.12, $k>4$, so we adopt the notation of 7.5. By 7.5.5, $J_{1}:=J \cap D_{1}$ is transitive on $\sigma=\sigma_{1} \in \Sigma$.

Let $\mathcal{C}$ be the connected component of $\Lambda^{\prime}$ containing $\bar{D}$. By 7.10, $\bar{D} \in \mathcal{W}_{0}^{!}$, so there exists a unique $U \in \mathcal{C} \cap \mathfrak{W}_{0}^{*}$ with $U \not \subset \bar{D}$. By 7.9, $Y:=U H$ is imprimitive. By 7.12.3, for each $\Gamma \in \mathcal{P}^{\prime}(Y), \Gamma \leq \Sigma$. Thus $\mathcal{P}^{\prime}(Y)$ has a unique maximal member $\Gamma$. As $\Gamma \leq \Sigma, \sigma \subseteq \gamma \in \Gamma$. By 3.8.1, $U$ does not act on $D$, so $\Gamma<\Sigma$. Then by $7.12 .2, \Gamma \in \mathcal{P}_{1}$, so $H_{*} \leq D(\Gamma)$.

As $\Gamma$ is maximal in $\mathcal{P}^{\prime}(Y), Y^{\gamma}$ is primitive. By 6.5.4, $Y^{\gamma}$ is not almost simple. As $J_{1}^{\gamma} \leq Y^{\gamma}$ and $J_{1}$ is transitive on $\sigma$, it follows from 3.5 in [8] that $k(\Gamma)=2 k$, 
$k=2^{a}, Y^{\gamma}$ is the stabilizer of an affine structure on $\gamma$, and $J_{1}^{\gamma} \not F^{*}\left(Y^{\gamma}\right)$. As $J_{1} \leq U$, it follows that

$$
X=\left\langle J_{1}^{N_{Y}(\gamma)}\right\rangle \leq U \cap B(\gamma),
$$

where $B(\gamma)=\hat{G}_{\Omega-\gamma}$, and that $F^{*}\left(Y^{\gamma}\right) \leq X^{\gamma}$. Therefore as $J_{1}^{\gamma} \not F^{*}\left(Y^{\gamma}\right)$ and $Y^{\gamma} / F^{*}\left(Y^{\gamma}\right)$ is simple, $X^{\gamma}=Y^{\gamma}$. In particular as $H_{*} \leq D(\Gamma), H_{*}^{\gamma} \leq X^{\gamma}$. Thus $H_{*} \leq\left\langle X^{H}\right\rangle \leq U$, a contradiction. This establishes the lemma.

Notation 7.14. By Hypothesis 7.1, $\mathcal{P}_{2}(H) \neq \varnothing$. In the remainder of the section, let $\Sigma \in \mathcal{P}_{2}(H), D:=D(\Sigma)$, and $W=: \bar{D}$. By 7.13, we have $k(\Sigma)=2$, so $\Sigma$ is maximal in $\mathcal{P}^{\prime}(H)$. By 7.10, $W \in W^{\text {! }}$. Let $\mathcal{C}$ be the connected component of $\Lambda^{\prime}$ containing $W$. As $W \in W^{!}$, there is a unique $U \in \mathcal{C} \cap W_{0}^{*}$ such that $U \not Z W$. Set $\mathcal{C}^{!}:=\mathcal{C} \cap w_{0}^{!}$, and set $s:=\left|\mathcal{C}^{!}-\{W\}\right|$. Thus $U$ is contained in each member of $\ell^{!}-\{W\}$.

Let $\left(s\right.$ be the set of pairs $(V, \Gamma)$ such that $V \in \mathcal{C}^{!}-\{W\}$ and $\Gamma \in \mathcal{P}^{\prime}(V H)$. Let $\mathfrak{V}$ consist of those $V \in \mathcal{C}^{!}-\{W\}$ such that $m_{2}(V \cap D) \geq m_{2}(D) / 2$. Let $z$ be the fixed-point-free involution in $S$ such that $\Sigma$ is the set of orbits of $Z:=\langle z\rangle$ on $\Omega$.

7.15. $\mathcal{P}_{2}(U H)=\varnothing$.

Proof. Suppose $\Gamma \in \mathcal{P}_{2}(U H)$, and let $E:=D(\Gamma)$. By 7.13, we have $k(\Gamma)=2$ and $\bar{E} \in W_{0}^{!}$by 7.10 .

As $k(\Gamma)=2, E$ is solvable. Then by 2.13 applied to $U, E, 1$ in the roles of $W, X, Y, U E \in W_{0}$. Therefore as $\bar{E} \in W_{0}^{!}, U E=\bar{E}$. Then $U \leq \bar{E}$, so $\bar{E} \in \mathcal{C}$. Then as $W, \bar{E} \in \mathcal{W}_{0}$, $W \cap \bar{E}=: V \in \mathcal{W}_{0}^{\prime}$, and by 3.7, $E=(V \cap E)(U \cap E)$. As $E$ is abelian and $J$ acts on $U_{E}:=U \cap E$ and $J_{E}=J \cap E, V=(V \cap E) J$ acts on $U_{E}$ and $J_{E}$. From 7.8, $H$ is irreducible on $U_{E} / J_{E}$, so as $V \cap D$ is an $H$-invariant 2-group, $V \cap D$ centralizes $U_{E} / J_{E}$. Thus $\left[U_{E}, V \cap D\right] \leq J_{E} \leq V \cap E$. Thus $E=(V \cap E) U_{E}$ acts on $Y=(V \cap E)(V \cap D)$. By symmetry, $D$ acts on $Y$, so $Y \unlhd M=\langle D, E, H\rangle$. However this contradicts 3.8.1.

7.16. For each $(V, \Gamma) \in$ (5, $\Gamma \in \mathcal{P}_{1}$.

Proof. As $U \leq V$, this follows from 7.15.

7.17. Assume $(V, \Gamma) \in \circlearrowleft$ such that $\Gamma \nless \Sigma \Sigma$. Then:

(1) $\Sigma \vee \Gamma=\infty$.

(2) $G_{\Gamma} \cap G_{\Sigma}=1$.

(3) $\left[H_{\Gamma}, V \cap D\right]=1$.

(4) $H_{*}$ centralizes $V \cap D$.

(5) $m_{2}(V \cap D) \leq n / 10$. 
Proof. Part (1) follows from the maximality of $\Sigma$ in $\mathcal{P}^{\prime}(H)$. Then (2) follows from (1) and 1.2, and (2) implies (3). By 7.16, we have $\Gamma \in \mathcal{P}_{1}$, so $H_{*} \leq H_{\Gamma}$. Thus (3) implies (4).

As $k(\Sigma)=2$,

$$
D \leq E=S_{\Sigma}=E_{1} \times \cdots \times E_{m},
$$

where $m=n / 2$ and $E_{i}=\left\langle e_{i}\right\rangle=S_{\Omega-\sigma_{i}} \cong Z_{2}$. Further $|E: D| \leq 2, H_{*}$ acts on $\Sigma$ with orbits $\left\{\Sigma_{1}, \ldots, \Sigma_{r}\right\}$ of length $s \geq 5$, and $C_{E}\left(H_{*}\right)=\left\langle f_{1}, \ldots, f_{r}\right\rangle$, where

$$
f_{j}=\prod_{i \in \Sigma_{j}} e_{i}
$$

is of order 2. Thus $m_{2}\left(C_{E}\left(H_{*}\right)\right)=r=m / s \leq m / 5=n / 10$. Then (4) completes the proof of (5).

7.18. Suppose $(V, \Gamma) \in$ G and $V \in \mathfrak{V}$.

(1) $m_{2}(V \cap D) \geq(n-2) / 4$.

(2) $\Gamma \leq \Sigma$, so $D \leq \hat{G}_{\Gamma}$.

(3) $H_{*}$ is nontrivial on $\Sigma_{\gamma}$ for each $\gamma \in \Gamma$.

(4) $k(\Gamma) \geq 10$ is even.

Proof. As $V \in \mathfrak{V}, m_{2}(V \cap D) \geq m_{2}(D) / 2$. As $k(\Sigma)=2, m_{2}(D) \geq(n-2) / 2$, so (1) holds. By (1), $m_{2}(V \cap D)>n / 10$, so (2) follows from 7.17.5.

By 7.16, $\Gamma \in \mathcal{P}_{1}$, so $H_{*} \leq G_{\Gamma}$ and then as $H$ is transitive on $\Gamma, H_{*}$ is nontrivial on $\gamma$. As $H$ acts on $\Sigma$ and $\Gamma \leq \Sigma, H_{*}$ acts on $\Sigma_{\gamma}$ and then as $k(\Sigma)=2$ and $H^{*}$ is perfect, (3) holds with $\left|\Sigma_{\gamma}\right| \geq 5$ and then (4) also follows.

7.19. $\mathfrak{V} \neq \varnothing$.

Proof. Adopt the notation of 7.8. As $W \in \mathcal{C}^{!}, W_{0}^{*} \cap W=\left\{U_{1}, \ldots, U_{s}\right\}$ is of order $s \geq 2$, so

$$
D^{*}=X_{1}^{*} \oplus \cdots \oplus X_{s}^{*}
$$

by 7.8, where $X_{i}:=U_{i} \cap D$. Order the $U_{i}$ so that $m_{2}\left(U_{i}\right) \geq m_{2}\left(U_{i+1}\right)$ for each $i$, and take $V \in \mathcal{C}^{!}-\{W\}$ such that $V \cap W=U_{1} \cdots U_{s-1}$. Thus

$$
m_{2}(V \cap D) \geq m_{2}(D) / 2 .
$$

7.20. Assume $V \in \mathfrak{V}$ and $Y:=V H$ is imprimitive on $\Omega$. Let $\Gamma$ be a maximal member of $\mathcal{P}^{\prime}(Y), \gamma \in \Gamma, r:=k(\Gamma)$, and $X:=V \cap D$. Then:

(1) $Y^{\gamma}$ is primitive but not almost simple.

(2) $r \equiv 0 \bmod 4$. 
(3) $z \in J$.

(4) $X \leq \hat{G}_{\Gamma}$ and $m_{2}\left(X^{\gamma}\right)>(r-2) / 4$.

(5) If $r=16$ and $m_{2}\left(X^{\gamma}\right)=4$, then $X$ is the direct product of the groups $X_{\Omega-\gamma}$, for $\gamma \in \Gamma$, with $X_{\Omega-\gamma} \cong E_{16}$.

Proof. By 7.16, we have $\Gamma \in \mathcal{P}_{1}$. By the maximality of $\Gamma, Y^{\gamma}$ is primitive. By 7.18.2, $X \leq V_{\Gamma}$, and by 7.18.1, $m_{2}(X) \geq(n-2) / 4$, so $X \neq 1$. Hence $Y^{\gamma}$ is not almost simple by 6.5 .4 , completing the proof of (1).

By 7.18.4, $r$ is even. Then (2) follows from (1) and 1.4. By (2), $z \in A$, so $z \in D$. Further $H$ centralizes $Z$, so (3) follows from 7.8.

As $X \leq V_{\Gamma}$ is $H$-invariant,

$$
m_{2}(X) \leq(n / r) \cdot m_{2}\left(X^{\gamma}\right)
$$

so as $m_{2}(X) \geq(n-2) / 4, m_{2}\left(X^{\gamma}\right) \geq(n-2) r / 4 n>(r-2) / 4$, establishing (4). Finally if $r=16$ and $m_{2}\left(X^{\gamma}\right) \leq 4$, then $m_{2}(X) \leq n / 4$, while as $n \equiv 0 \bmod 4$, $n / 4$ is the smallest integer as big as $(n-2) / 4$. We conclude that $m_{2}(X)=n / 4$ and then that (5) holds.

7.21. For each $V \in \mathfrak{V}, V H$ is primitive on $\Omega$.

Proof. Assume otherwise and choose notation as in 7.20. We observe that $Y^{\gamma}, \gamma$, $z^{\gamma}, X^{\gamma}$ satisfy the hypotheses of 1.9 in the role of $G, \Omega, z, X$ by 7.20. Then we conclude from 1.9 that $r=16, Y^{\gamma}$ is affine and $X^{\gamma} \cong E_{16}$. Then by 7.20.5, $X$ is a direct product of the groups $X_{\Omega-\gamma}$. Hence by $1.9, Y_{\Gamma}=\left\langle X^{Y}\right\rangle$, so $Y$ is the direct product of the groups $Y_{\Omega-\gamma} \cong Y^{\gamma}$. As $X \leq V \unlhd Y, Y_{\gamma} \leq V$. But then as $H_{*}^{\gamma} \leq Y^{\gamma}$ for each $\gamma \in \Gamma, H_{*} \leq V$, a contradiction.

7.22. Let $V \in \mathfrak{V}$ and $Y:=V H$. Then:

(1) $Y$ is primitive but not almost simple on $\Omega$.

(2) $n \equiv 0 \bmod 4$.

(3) $z \in J$.

Proof. By 7.21, $Y$ is primitive on $\Omega$, so (1) follows from 2.14. As $k(\Sigma)=2, n$ is even, so as $Y$ is primitive but not almost simple, (2) follows from 1.4. Now (3) follows as in the proof of 7.20.3.

We are now in a position to obtain a contradiction to Hypothesis 7.1. We argue as in the proof of 7.21. By 7.19 we can pick $V \in \mathfrak{V}$; set $Y:=V H$ and $X:=V \cap D$. 
By 7.18.1, $m_{2}(X) \geq(n-2) / 4$. Then by 7.22 , the hypotheses of 1.9 are satisfied by $Y$ in the role of $G$, so that lemma says that $n=16$ and $Y=\left\langle X^{Y}\right\rangle$. Then as $X \leq V \unlhd Y, H_{*} \leq Y=V$, for our final contradiction.

This contradiction shows:

Theorem 7.23. Assume Hypothesis 6.1. Then $\mathcal{P}_{2}(H)=\varnothing$, so $\mathcal{P}^{\prime}(H)=\mathcal{P}_{1}(H)$.

\section{The case $\mathscr{P}^{\prime}(H)=\mathscr{P}_{1}$}

In this section we continue to assume Hypothesis 6.1, and adopt the notation established there.

8.1. The following hold:

(1) $\mathcal{P}^{\prime}(H)=\mathcal{P}_{1}(H)$.

(2) Let $\Gamma$ be the set of orbits of $H_{*}$ on $\Omega$. Then $\Gamma$ is the greatest member of $\mathcal{P}^{\prime}(H)$.

(3) For each $\gamma \in \Gamma, H^{\gamma}$ is primitive.

(4) If $Y \in \mathcal{O}_{G}(H)$ is imprimitive, then there is a greatest member $\Gamma_{Y}$ of $\mathcal{P}^{\prime}(Y)$, and $\Gamma_{Y} \leq \Gamma$.

Proof. Part (1) is a restatement of Theorem 7.23. Then (2) follows from parts (1) and (2) of 6.5. As $\Gamma$ is maximal in $\mathcal{P}^{\prime}(H),(3)$ and (4) follow.

8.2. Assume that for $\gamma \in \Gamma$ that $J_{\Omega-\gamma} \neq 1$. Then for each $W \in W_{0}^{\prime}$,

(1) WH is imprimitive on $\Omega$.

(2) $\mathcal{P}^{\prime}(W H)$ has a greatest member $\Gamma_{W}$, and $\Gamma_{W} \leq \Gamma$.

Proof. Assume $I:=J_{\Omega-\gamma} \neq 1$. Then as $I^{\gamma} \unlhd H^{\gamma}$, it follows that $I^{\gamma}$ is transitive by 8.1.3. Let $W \in \mathcal{W}_{0}$ and $Y:=W H$. Suppose $Y$ is primitive on $\Omega$. Then by 3.5 in [8] and 2.14, $Y$ is the stabilizer of an affine structure on $\Omega, J \not \leq F^{*}(Y)$, and $Y=\left\langle F^{*}(Y), J^{Y}\right\rangle$. As $F^{*}(Y)$ is the unique minimal normal subgroup of $Y$ and $1 \neq W \unlhd Y, F^{*}(Y) \leq W$. Then as $J \leq W, Y=\left\langle F^{*}(Y), J^{Y}\right\rangle \leq W$. But now $H_{*} \leq Y \leq W$, a contradiction. Thus (1) holds. Then (1) and 8.1.4 imply (2) with $\Gamma_{W}=\Gamma_{Y}$.

8.3. For each $\gamma \in \Gamma, J_{\Omega-\gamma}=1$.

Proof. Assume otherwise. By 3.3, there exist $W \in W_{0}^{\prime}$ with $Y:=W H \not \leq M(\Gamma)$. Set $\Sigma:=\Gamma_{W}$; by 8.2, $\Sigma<\Gamma$. Let $\gamma \subseteq \sigma \in \Sigma, I:=J_{\Omega-\gamma}$ and $X:=\left\langle I^{N_{Y}(\sigma)}\right\rangle$. Then $X \leq W$. By maximality of $\Sigma$ in $\mathcal{P}^{\prime}(Y), Y^{\sigma}$ is primitive. Hence by 3.5 in 
[8] and 6.5.4, $Y^{\sigma}$ is the stabilizer of an affine structure on $\sigma, I^{\sigma} \not F^{*}\left(Y^{\sigma}\right)$, and $Y^{\sigma}=\left\langle F^{*}\left(Y^{\sigma}\right), I^{\sigma Y^{\sigma}}\right\rangle$. As $F^{*}\left(Y^{\sigma}\right)$ is the unique minimal normal subgroup of $Y^{\sigma}$ and $1 \neq X^{\sigma} \unlhd Y^{\sigma}, F^{*}\left(Y^{\sigma}\right) \leq X^{\sigma}$. Then as $I \leq X$,

$$
Y^{\sigma}=\left\langle F^{*}\left(Y^{\sigma}\right), I^{\sigma Y^{\sigma}}\right\rangle \leq X^{\sigma} .
$$

Now as $X \leq W_{\Omega-\sigma}$ and $H_{*}^{\sigma} \leq Y^{\sigma}, H_{*} \leq\left\langle X^{H}\right\rangle \leq W$, a contradiction.

In the remainder of this section (until the last theorem) we assume the following hypothesis:

Hypothesis 8.4. $J_{E}:=J \cap D(\Gamma) \neq 1$.

In addition adopt the following notation: Set $M:=M(\Gamma), E:=D(\Gamma)$, and for $\gamma \in \Gamma$, let $E(\gamma):=A_{\Omega-\gamma}$ (a component of $E$ ), $\pi_{\gamma}: E \rightarrow E(\gamma)$ the projection map, $X_{\gamma} \leq J_{E} \pi_{\gamma}$ such that $X_{\gamma}^{\gamma}$ is a minimal normal subgroup of $H^{\gamma}$ and $H$ permutes $\left\{X_{\gamma}: \gamma \in \Gamma\right\}$ via conjugation, and $X:=\left\langle X_{\gamma}: \gamma \in \Gamma\right\rangle$. Set $U:=\bar{X}$ and $k=k(\Gamma):=|\gamma|$.

8.5. Let $\gamma \in \Gamma$. Then:

(1) $H^{\gamma}$ is primitive and $X_{\gamma} \cong X_{\gamma}^{\gamma}$ is transitive on $\gamma$.

(2) $U \in W_{0}^{!}$.

(3) Let $\mathcal{W}_{0}^{*} \cap U=\{U(i): 1 \leq i \leq s\}$ and for $\alpha \subseteq\{1, \ldots, s\}$, set

$$
U(\alpha):=\langle U(i): i \in \alpha\rangle \text { and } X(\alpha):=U(\alpha) \cap X .
$$

Then $U(\alpha)=\bar{X}(\alpha), X(\alpha)=\langle X(i): i \in \alpha\rangle$, and $\bigcap_{i} X(i)=J \cap X=: J_{X}$.

(4) For $\gamma \in \Gamma$ and $1 \leq i \leq s, J_{X} \pi_{\gamma}=X(i) \pi_{\gamma}=X_{\gamma}$.

(5) $\mathcal{W}_{0}(\leq U)=\Xi_{M}$.

Proof. Observe that Hypothesis 4.1 is satisfied by $M:=M(\Gamma)$ and

$$
\mathscr{L}:=\{E(\gamma): \gamma \in \Gamma\} .
$$

Further in the language of Notation 4.2, in this setup, $X=\tilde{J}_{X}$, where $J_{X}$ is the preimage in $J_{E}$ under $\pi_{\gamma}$ of a minimal normal subgroup of $H^{\gamma}$. Moreover we have $J_{X}=J \cap X$. By 4.4.2, $\tilde{J} J \in \mathcal{W}_{0}$, so as $X \leq \tilde{J}$ is $H$-invariant, $U=\bar{X} \in \mathcal{W}_{0}$ by 2.7. By 8.2.3, $H^{\gamma}$ is primitive, so as $1 \neq X_{\gamma}^{\bar{\gamma}} \unlhd H^{\gamma}, X_{\gamma}$ is transitive on $\gamma$. Thus (1) holds, and if $U \leq W \in \mathcal{W}_{0}$, then arguing as in the proof of 8.2, $Y:=W H$ is imprimitive on $\Omega$, there is a greatest member $\Gamma_{W}$ of $\mathcal{P}^{\prime}(Y)$, and $\Gamma_{W} \leq \Gamma$. Then arguing as in the proof of 8.3, $\Gamma_{W}=\Gamma$. 
Suppose (2) fails, and let $\left\{W_{i}: 1 \leq i \leq s\right\}=\left\{W \in W^{!}: U \leq W\right\}$. By the previous paragraph, $W_{i} \leq M$. Thus the connected component $\ell$ of $U$ is contained in $\Xi_{M}$, so by $4.6 .4, X \leq J$, contrary to 8.3 .

Therefore (2) holds. Thus as $U=\bar{X}$, we conclude from 3.7 that (3) holds. By construction of $X, J_{X} \pi_{\gamma}=X_{\gamma}=X \pi_{\gamma}$, so as $J_{X}=J \cap X \leq X(i) \leq X$, part (4) follows from (3).

Recall $X=\tilde{J}_{X}$ and by 8.3, $X \not \leq J$. Thus from 4.6.3, $\Xi_{M}$ is connected, but does not contain the connected component of $U$. Thus (5) follows from (2).

8.6. Let $\gamma \in \Gamma$. Then either:

(1) $k=p^{a}$ is a prime power, $H^{\gamma}$ is affine, and $X_{\gamma} \cong X^{\gamma}=F^{*}\left(H^{\gamma}\right) \cong E_{p^{a}}$ is regular on $\gamma$. Further $X=X(1) \cdots X(s)$, and for each $1 \leq i \leq s, X(i)$ is normal in $U H$.

(2) $X^{\gamma}$ is the direct product of components transitively permuted by $H^{\gamma}$ isomorphic to some simple group $X_{0}$, and $X$ is the direct product of a set $X$ of components transitively permutated by $H$ and isomorphic to $X_{0}$. For $\alpha \subseteq\{1, \ldots, s\}$ there exist an $H$-invariant partition $\Theta(\alpha)$ of $\mathcal{X}$ such that $X(\alpha)$ is the direct product of full diagonal subgroups of $X_{\theta}, \theta \in \Theta(\alpha)$, and $X_{\theta}=\prod_{Y \in \theta} Y$. Thus $X(\alpha)$ is the direct product of its components, each of which is isomorphic to $X_{0}$, and $H$ is transitive on these components.

Proof. As $X_{\gamma} \cong X_{\gamma}^{\gamma}$ is a minimal normal subgroup of the primitive group $H^{\gamma}$, either

(i) $k=p^{a}$ is a prime power, $H^{\gamma}$ is affine, and $X_{\gamma}^{\gamma}=F^{*}\left(H^{\gamma}\right) \cong E_{p^{e}}$ is regular on $\gamma$, or

(ii) $X_{\gamma}^{\gamma}$ is the direct product of components permuted transitively by $H^{\gamma}$ and isomorphic to some nonabelian simple group $X_{0}$.

Then as $H$ is transitive on $\Gamma$ and $X$ is the direct product of the groups $X_{\gamma}, \gamma \in \Gamma$, it follows that $X$ is an elementary abelian $p$-group in (i), while in (ii), $X$ is the direct product of its set $\mathcal{X}$ of components, with $H$ transitive on $\mathcal{X}$ and the members of $\mathcal{X}$ are isomorphic to $X_{0}$. Then in (i), (1) holds by 8.5.3. Similarly in (ii), (2) holds using 8.5.4 and 1.4 in [7].

Notation 8.7. Assume Hypothesis 8.4, adopt the notation of 8.5.3, and let $\mathcal{C}$ be the connected component of $\Lambda^{\prime}$ containing $U$. By 8.5.2, there is a unique $V \in \mathcal{W}_{0}^{*} \cap \mathcal{C}$ with $V \not \leq U$. Let

$$
W^{!} \cap \mathcal{C}=: \mathcal{C}^{!} \text {and } \mathcal{V}:=\{W \in \mathcal{C}: W \not U U\} .
$$

Thus $\mathcal{C}^{!}-\{U\} \subseteq \mathcal{V}$, and for each $W \in \mathcal{V}, V \leq W$. 
For $W \in \mathcal{V}$, set $Y_{W}=W H$. If $Y_{W}$ is primitive on $\Omega$, let $\Gamma_{W}=0$ and $\Phi_{W}=\Omega$. If $Y_{W}$ is not primitive on $\Omega$, then by 8.1.4 there is a greatest member $\Gamma_{W}=\Gamma_{Y_{W}}$ of $\mathcal{P}^{\prime}\left(Y_{W}\right)$, and we have $\Gamma_{W} \leq \Gamma$. In this case let $\Phi_{W} \in \Gamma_{W}$. Set $B_{W}=F^{*}\left(Y_{W}\right)$ if $\Phi_{W}=\Omega$, and let $B_{W}$ be the product of the $H$-conjugates of the inverse image in $A_{\Omega-\Phi_{W}}$ of $F^{*}\left(Y_{W}\right)^{\Phi_{W}}$ if $\Phi_{W} \neq \Omega$.

8.8. Assume Hypothesis 8.4 and adopt Notation 8.7. Let $W \in \mathcal{V}$. Then:

(1) $Y_{W}^{\Phi_{W}}$ is primitive but not almost simple.

(2) $\Gamma_{\Phi_{W}}$ is a $(U H)^{\Phi_{W}}$ invariant partition of $\Phi_{W}$.

(3) Suppose $\Phi_{W} \neq \Omega$. Let $\alpha \in \Gamma_{W}$, and $\pi_{\alpha}: D\left(\Gamma_{W}\right) \rightarrow A_{\Omega-\alpha}$ be the projection. Then $D(\Gamma) \leq D\left(\Gamma_{W}\right)$, so $\langle U, W\rangle \leq M\left(\Gamma_{W}\right)$. Further for each $P \in \Xi_{M\left(\Gamma_{W}\right)}$, $\left(P \cap D\left(\Gamma_{W}\right)\right) \pi_{\alpha} \leq P$.

(4) $B_{W} \leq W$.

(5) $B_{W} \not U$.

(6) If $W \notin W_{0}^{!}$, then $B_{W} \neq B_{P}$ for some $P \in \mathcal{W}_{0}$ with $W$ maximal in $P$.

(7) Suppose $V \leq Q \leq W$ with $Q \in \mathcal{W}_{0}$. Then $\Gamma_{Q}=\Gamma_{W}, Y_{Q}^{\Phi_{W}}$ is primitive, and $B_{Q} \leq B_{W}$.

(8) There exists $W \in \mathcal{C}$ ! and $V=V_{1}<\cdots<V_{s}=W$ with $V_{i} \in \mathcal{W}_{0}$ and $\bar{B}_{V_{i}}=V_{i}$ for each $1 \leq i \leq s$.

Proof. Let $\Phi:=\Phi_{W}, Y:=Y_{W}, B:=B_{W}$. If $\Phi=\Omega$, then, by definition of $\Phi$, $Y$ is primitive on $\Omega$, so $Y^{\Phi}$ is primitive, while $Y^{\Phi}$ is not almost simple by 2.14 . If $\Phi \neq \Omega$, then $Y^{\Phi}$ is primitive as $\Phi \in \Gamma_{W}$ and $\Gamma_{W}$ is the greatest member of $\mathcal{P}^{\prime}(Y)$. Further $Y^{\Phi}$ is not almost simple by 8.1 .1 and 6.5.4. Thus (1) is established.

As $\Gamma \in \mathcal{P}^{\prime}(H)$, and $\Gamma_{W} \leq \Gamma$ when $\Phi \neq \Omega$, (2) follows.

Assume the setup of (3). By 8.1.4, $\Gamma_{W} \leq \Gamma$, so $D(\Gamma) \leq D\left(\Gamma_{W}\right)$. Therefore $X \leq D(\Gamma) \leq M\left(\Gamma_{W}\right)=: M_{W}$, so $U=J X \leq M_{W}$, and hence $\langle U, W\rangle \leq M_{W}$ as $\Gamma_{W} \in \mathcal{P}^{\prime}(H W)$. Observe that Hypothesis 4.1 is satisfied by $M_{W}, D\left(\Gamma_{W}\right)$ in the roles of " $M, D$ ". Further as $\langle U, W\rangle \leq M_{W}$, each group in the connected component of $U$ is contained in $M_{W}$. Therefore (3) follows from 4.6.4.

By definition in 8.7, $B^{\Phi}=F^{*}\left(Y^{\Phi}\right)$. Hence by (1), either $B^{\Phi}$ is the unique minimal normal subgroup of $Y^{\Phi}$, or $Y^{\Phi}$ is doubled. As $1 \neq W^{\Phi} \unlhd Y^{\Phi}$, we conclude that either $B^{\Phi} \leq W^{\Phi}$ or $Y^{\Phi}$ is doubled, with $B^{\Phi}=F_{1}^{\Phi} \times \bar{F}_{2}^{\Phi}$ the product of the two minimal normal subgroups $F_{j}^{\Phi}$ of $Y^{\Phi}$, and $F_{1}^{\Phi}=B^{\Phi} \cap W^{\Phi}$. Now in the second case, as

$$
H_{*} W / W=F^{*}(Y / W) \cong L
$$


it follows that $W^{\Phi} H_{*}^{\Phi}=W^{\Phi} F_{2}^{\Phi}$, and then $F_{1}^{\Phi} \cong F_{2}^{\Phi} \cong L$. Also

$$
\left[F_{2}^{\Phi}, W^{\Phi}\right] \leq F_{2}^{\Phi} \cap W^{\Phi}=1,
$$

so $F_{2}^{\Phi}$ centralizes $W^{\Phi}$. Therefore if $H_{*}^{\Phi}=F_{2}^{\Phi}$ then $H_{*}$ centralizes $W$. But then by 6.5.2, $W \leq M$, contrary to 8.5.5. Thus $H_{*}^{\Phi^{2}} \neq F_{2}^{\Phi}$, so $\bar{F}_{2} \in \mathcal{W}_{0}$ by 2.12 , where $F_{2}$ is the product of the $H$-conjugates of the inverse image of $F_{2}^{\Phi}$ in $A_{\Omega-\Phi}$ in case $\Phi \neq \Omega$. Further $\left[F_{2}, W\right]=1$, so as $J_{X} \leq W$ and as $\Gamma$ is the set of orbits of $J_{X}$ on $\Omega$, we have $F_{2} \leq M$. Thus $\bar{F}_{2} \leq U$ by 8.5.5. We saw that $H$ is transitive on the components of $F_{2}$, so $F_{2}$ is a minimal normal subgroup of $F_{2} H$. Therefore, in the notation of 8.5.3, $F_{2} \leq X(i)$ for some $1 \leq i \leq s$, and then from 8.5.1 and 8.5.4, $\Gamma$ is the set of orbits of $F_{2}$ on $\Omega$. As $W$ centralizes $F_{2}$, it follows that $W \leq M$, contradicting 8.5.5.

Thus $B^{\Phi} \leq W^{\Phi}$. Then (4) is immediate from the definition of $B$ if $\Phi=\Omega$, while if $\Phi \neq \Omega$, it follows from (3).

Assume $B \leq U$. Claim $T:=X \cap B \neq 1$. For if not, $[X \cap W, B] \leq X \cap B=1$, so $X \cap W \leq \bar{C}_{W}(B) \leq B$ as $B^{\Phi}=F^{*}\left(Y^{\Phi}\right)$. In particular

$$
1 \neq J_{X} \leq X \cap W \leq T
$$

establishing the claim.

Now, using (4), $B \leq U \cap W \leq N_{W}(X) \leq N_{W}(T)$. Also $T$ is $H$-invariant, so $\bar{T} \in \mathcal{W}_{0}$ by 2.7 , and as $T \neq 1, T^{\gamma}$ is transitive by 8.5 .1, so $\Gamma$ is the set of orbits of $T$ on $\Omega$. Adopt the notation of 3.7 with $B, Y$ in the roles of $Y, X$. Then we have $\mathscr{D}=\mathcal{W}_{0}(\leq W)$ and $\bar{T}=L_{\alpha}$ for some $\alpha \subseteq \mathcal{A}$, so by 3.7, $L_{\mathcal{B}}$ acts on $T=L_{\alpha} \cap B$, so $T$ is normal in $\left\langle B, L_{\mathscr{B}}\right\rangle=\left\langle L_{\mathcal{A}}, L_{\mathscr{B}}\right\rangle=L_{\mathscr{D}}=W$. Therefore $W$ acts on the set $\Gamma$ of orbits of $T$, so $W \leq M$, contrary to 8.5.5. This completes the proof of (5).

Assume (6) fails and let $\mathscr{D}:=\mathcal{C} \cap W_{0}^{*}$. From 3.4, $W=L_{\alpha}$ for some $\alpha \subseteq \mathscr{D}$, and the members of $\mathcal{W}_{0}$ in which $W$ is maximal are the groups $L(b)=L_{\alpha \cup\{b\}}$, $b \in \mathscr{B}=\mathscr{D}-\alpha$. By hypothesis, $B=B_{L(b)}$ for each $b \in \mathscr{B}$, so $L_{\mathscr{D}}$ acts on $B$, contrary to 3.8. This establishes (6).

Assume the hypothesis of (7). By (3), $U$ acts on $\Gamma_{Q}$, so $W \leq\langle U, Q\rangle$ acts on $\Gamma_{Q}$, and then by definition of $\Gamma_{W}, \Gamma_{W}=\Gamma_{Q}$. Therefore also $\Phi_{W}=\Phi_{Q}$, so $Y_{Q}^{\Phi_{W}}$ is primitive by (1). By (5), $\bar{B}$ and $\bar{B}_{V}$ are not contained in $U$, while $\bar{B}_{V} \leq V$ by (4), so as $V \cap U=J$ and $V \in W_{0}^{*}, \bar{B}_{V}=V$, and by 3.7, $V \cap B \not J$. Thus $Q \cap B \neq 1$, so

$$
1 \neq Q^{\Phi} \cap B^{\Phi} \unlhd Y_{Q}^{\Phi} .
$$

Then as $B_{Q}^{\Phi}=F^{*}\left(Y_{Q}^{\Phi}\right)$ and $Y_{Q} \leq Y$,

$$
1 \neq B_{Q}^{\Phi} \cap B^{\Phi}
$$

Then by 4.3 in [4], $B_{Q}^{\Phi} \leq B^{\Phi}$, so $B_{Q} \leq B$, completing the proof of (7). 
We saw $\bar{B}_{V}=V$. Let $V=V_{1}<\cdots<V_{r}=W$ be a chain of maximal length subject to $\bar{B}_{V_{i}}=V_{i}$, and suppose $W \notin W_{0}$. Then for $P \in W_{0}$ with $W$ maximal in $P, \bar{B}_{P} \neq P$. However by (7), $B \leq B_{P}$, so $W=\bar{B} \leq \bar{B}_{P}$, and hence $W=\bar{B}_{P}$ by maximality of $W$ in $P$. This contradicts (6), and completes the proof of (8).

8.9. Choose $W$ as in 8.8.8 and set $Y:=Y_{W}, \Phi:=\Phi_{W}$, and $B:=B_{W}$. Then:

(1) $B=\left\langle B \cap U, B_{V}\right\rangle$ and

$$
(B \cap U) \cap B_{V}=B \cap J
$$

with $\overline{B \cap U}$ maximal in $W_{0}(<U)$ and $W_{0}(<W), \bar{B}_{V}=V$, and $\bar{B}=W$.

(2) $Y=B H$.

(3) $Y^{\Phi}$ is not affine.

(4) $Y_{V}^{\Phi}$ is primitive.

(5) $Y^{\Phi}$ is semisimple, and hence $Y^{\Phi}$ preserves a regular product structure $\mathcal{F}$ on $\Phi$. Further $B^{\Phi}$ is the direct product of the set $\mathscr{L}$ of components of $Y^{\Phi}$, each of which is simple, and $\mathscr{L}$ is permuted transitively by $H^{\Phi}$.

(6) $H_{*}$ acts nontrivially on $\mathscr{L}$.

Proof. First 8.8.8 and 3.7 imply (1). By (1), $W=\bar{B}=B J$, so

$$
Y=W H=B J H=B H,
$$

establishing (2).

Suppose $Y^{\Phi}$ is affine. Then $B^{\Phi}$ is an elementary abelian $p$-group for some prime $p$, and $Y^{\Phi}$ is irreducible on $B^{\Phi}$. But by (2), $Y=B H$, so $H^{\Phi}$ is irreducible on $B^{\Phi}$. However by (1), $B_{V}$ is a proper nontrivial subgroup of $B$, while by 8.8.7, $\Gamma_{V}=\Gamma_{W}$, so $B_{V}^{\Phi}$ is a proper nontrivial subgroup of $B^{\Phi}$. However $B_{V}^{\Phi}$ is $H^{\Phi}$-invariant, contradicting $H^{\Phi}$ irreducible on $B^{\Phi}$. Hence (3) holds.

Part (4) follows from 8.8.7.

By (1), $\bar{B}_{V}=V$ and $\bar{B}=W$, so as $V \neq W$ also $B_{V} \neq B$. From the proof of 8.8.7, $B_{V}^{\Phi} \leq B^{\Phi}$, so as $B_{V} \neq B$ we have $B_{V}^{\Phi}<B^{\phi}$.

By (3) and as $Y^{\Phi}$ is primitive, $B^{\Phi}$ is the direct product of a set $\mathscr{L}$ of simple components. Further $H^{\Phi}$ is transitive on $\mathscr{L}$ iff $Y^{\Phi}$ is not doubled.

Suppose $Y^{\Phi}$ is doubled. Then $B^{\Phi}=B_{1}^{\Phi} \times B_{2}^{\Phi}$ is the product of the two minimal normal subgroups of $Y^{\Phi}$. By (4) and Proposition 8 in [4], and as

$$
F^{*}\left(Y_{V}^{\Phi}\right)=B_{V}^{\Phi} \neq B^{\Phi}
$$

it follows that we may choose $B_{V}^{\Phi}=B_{1}^{\Phi}$. As $C_{Y_{V}^{\Phi}}\left(B_{V}\right)=1$,

$$
Y_{V}^{\Phi} \cap B^{\Phi}=B_{1}^{\Phi} \text {. }
$$


Now $B_{2}^{\Phi}$ is an $H^{\Phi}$-invariant subgroup of $B^{\Phi}$, so by $3.7, B_{2}^{\Phi} \leq U^{\Phi}$. But then $(U H)^{\Phi}$ is primitive, contradicting 8.8.2. Therefore $H^{\Phi}$ is transitive on $\mathscr{L}$ and $Y^{\Phi}$ is not doubled.

As $B_{V}^{\Phi}<B^{\Phi}, Y^{\Phi}$ is not complemented by Proposition 10 in [4]. By Proposition 6 in [4] and as $H^{\Phi}$ is transitive on $\mathscr{L}, Y^{\Phi}$ is not diagonal. Hence (5) holds.

By 8.1.2, $H_{*}$ is trivial on $\Gamma$, so as $\Gamma_{W} \leq \Gamma, H_{*} \leq Y_{\Gamma_{W}}$. By (1), $\bar{B}=W$, so $H_{*} \not \leq B$. Let $K$ be the kernel of the action of $Y$ on its components. Then $K / B$ is solvable, so as $H_{*} \not \subset B$, also $H_{*} \not \leq K$. Hence (6) follows from the transitivity of $H$ on the components of $Y$.

8.10. Choose $W$ as in 8.8.8, set $\Phi:=\Phi_{W}$, and let

$$
\mathcal{R}(\Phi):=\left\{g \in \operatorname{Sym}(\Phi):\left|\operatorname{Fix}_{\Phi}(g)\right| \geq|\Phi| / 2\right\} .
$$

Then:

(1) $U \cap W=U(\alpha)$ and $X \cap W=X(\alpha)$, for some maximal proper subset $\alpha$ of $\{1, \ldots, s\}$.

(2) Assume 8.6.2 holds. Then $X(\alpha)=\langle X(\alpha) \cap \mathcal{R}(\Phi)\rangle$.

Proof. Part (1) is a consequence of 8.5.3 and 3.4.

Assume 8.6.2 holds, adopt the notation of 8.6.2, and set

$$
\mathcal{X}^{\Phi}:=\left\{F^{\Phi}: F \in \mathcal{X} \text { and } F^{\Phi} \neq 1\right\} .
$$

For $\gamma \in \Gamma_{\Phi}$, set $\hat{\gamma}:=\left\{F^{\Phi} \in \mathcal{X}^{\Phi}: F^{\Phi} \leq E(\gamma)^{\Phi}\right\}$. Then $\hat{\Gamma}=\left\{\hat{\gamma}: \gamma \in \Gamma_{\Phi}\right\}$ is a partition of $\mathcal{X}^{\Phi}$ with

$$
|\hat{\Gamma}|=\left|\Gamma_{\Phi}\right|=: m \text {. }
$$

By 8.6, for each $\beta \subseteq \alpha, X(\beta)^{\Phi}$ is the direct product of components $F_{\theta}^{\Phi}$, where $\theta \in \Theta_{\beta, \Phi}$, such that $\Theta_{\beta, \Phi}$ is an $H^{\Phi}$-invariant partition of $\mathcal{X}^{\Phi}$, and $F_{\theta}^{\Phi}$ is a full diagonal subgroup of $X_{\theta}^{\Phi}$. As $F_{\theta}^{\Phi}$ is simple and $\pi_{\gamma}: X(\beta)^{\Phi} \rightarrow X_{\gamma}^{\Phi}$ is a surjection, $F_{\theta}^{\Phi} \pi_{\gamma}$ is a component of $X_{\gamma}^{\Phi}$ whenever $F_{\theta}^{\Phi} \pi_{\gamma} \neq 1$. Thus $\hat{\Gamma} \vee \Theta_{\beta, \Phi}=\infty$, and hence $k_{\beta}=|\theta| \leq m$. Now for $\delta \subseteq \beta, X(\delta) \leq X(\beta)$, so $\Theta_{\beta, \Phi} \leq \Theta_{\delta, \Phi}$. It follows that $m \geq k_{\varnothing} \geq 2^{s-1} k_{\alpha}$, so $k_{\alpha} \leq m / 2^{2-1} \leq m / 2$.

Take $\beta=\alpha$. Now $F_{\theta}^{\Phi} \leq A_{\Phi-\mu}$, where

$$
\mu=\bigcup_{\left\{\gamma: F_{\theta}^{\Phi} \pi_{\gamma} \neq 1\right\}} \gamma,
$$

and $\mu$ is of order $k k_{\alpha} \leq k m / 2=|\Phi| / 2$, so $F_{\theta}^{\#} \subseteq \mathcal{R}(\Phi)$, completing the proof of the lemma. 
8.11. Assume 8.6.1 holds and choose $W$ as in 8.8 .8 , set $Y:=Y_{W}, \Phi:=\Phi_{W}$, and $B:=B_{W}$, and let $K$ be the kernel of the action of $Y^{\Phi}$ on the product structure $\mathcal{F}$ of 8.9.5. Then:

(1) $(X \cap W)^{\Phi} \leq K$.

(2) $X_{B}:=(X \cap B)^{\Phi} \neq 1$.

(3) $X_{B}$ contains its projection on each member of $\mathscr{L}$.

Proof. By 8.10.1, $X \cap W=X(\alpha)$ for some maximal proper subset $\alpha$ of $\{1, \ldots, s\}$, and by 8.5.3, $U \cap W=U(\alpha)=\bar{X}(\alpha)$. Therefore $X \cap W \neq 1$.

Let $\gamma \in \Gamma, I:=(X \cap W)^{\Phi}$, and suppose $P$ is a nontrivial $H$-invariant subgroup of $C_{M^{\Phi}}(I)$. Then $1 \neq I^{\gamma} \unlhd H^{\gamma}$, so by 8.5.4, $I^{\gamma}=X^{\gamma}$. Thus

$$
P^{\gamma} \leq C_{M^{\gamma}}\left(X^{\gamma}\right)=X^{\gamma}
$$

so $P_{\Gamma_{\Phi}} \leq C_{M^{\Phi}}\left(X^{\Phi}\right)=X^{\Phi}$, and $N_{P}(\gamma) \leq C_{M^{\Phi}}\left(X_{\gamma}\right)$, so that

$$
X_{P}:=C_{X^{\Phi}}(P)=\prod_{\sigma \in \Sigma} X_{\sigma},
$$

where $\Sigma$ is the set of orbits for $P$ on $\Gamma_{\Phi}$ and $X_{\sigma}$ is a full diagonal subgroup of $\left\langle X_{\gamma}: \gamma \in \sigma\right\rangle$.

Suppose $P \not \leq X^{\Phi}$. Then $P \neq P_{\Gamma_{\Phi}}$, so $\Sigma \neq \Gamma_{\Phi}$, and hence $X_{P} \neq X^{\Phi}$. Hence $J_{X}^{\Phi} \leq(X \cap W)^{\Phi} \leq X_{P}<X^{\Phi}$, and $X_{P}$ is $H^{\Phi}$-invariant, so

$$
X_{P}=(X \cap W)^{\Phi}=I
$$

by 3.7 and maximality of $(X \cap W)^{\Phi}=X(\alpha)^{\Phi}$ in $X^{\Phi}$. Further either

(i) $P$ is transitive on $\Gamma_{\Phi}$, or

(ii) $\left(X_{\sigma}^{\Phi}\right)^{\#} \subseteq \mathcal{R}(\Phi)$, so $I=\left\langle X_{P} \cap \mathcal{R}(\Phi)\right\rangle \leq K$,

by 8.9.5 and 4.2 in [8]. Indeed if (i) holds, then $X_{P} \cong X_{\gamma}$ as a $N_{H}(\gamma)$-module, so $N_{H}(\gamma)$ is irreducible on $X_{P}$, and hence $X_{P}=J_{X}^{\Phi}$, contradicting

$$
J_{X}^{\Phi}<X(\alpha)^{\Phi}=X_{P}
$$

Thus (ii) holds if $P \not \leq X^{\Phi}$.

Claim $X_{B}:=I \cap B^{\Phi} \neq 1$. Assume otherwise. Then $\left[I,(B \cap U)^{\Phi}\right] \leq X_{B}=1$. Also $(B \cap U)^{\Phi} \neq 1$ by 8.9.1, so $(B \cap U)^{\Phi} \not \leq X^{\Phi}$ or else $(B \cap U)^{\Phi} \leq I \cap B^{\Phi}=1$. Thus applying remarks above to $(B \cap U)^{\Phi}$ in the role of $P$, we conclude (ii) holds, so $I \leq K$. Then as $I$ centralizes $(B \cap U)^{\Phi}$, it also centralizes the product $\tilde{B}$ of the projections of $(B \cap U)^{\Phi}$ on the various members of $\mathscr{L}$. Hence applying remarks above to $\tilde{B}$ in the role of $P, \tilde{B}_{\Gamma_{\Phi}} \leq X^{\Phi}$, so that $\tilde{B}_{\Gamma_{\Phi}} \leq X^{\Phi} \cap B^{\Phi}=X_{B}=1$. 
Then as $\left[H_{*}, \tilde{B}\right] \leq \tilde{B}_{\Gamma_{\Phi}}, H_{*}$ centralizes $\tilde{B}$, which is not the case as $H_{*}$ acts nontrivially on $\mathscr{L}$ by 8.9.6. Thus the claim is established, so (2) holds.

Next let $\tilde{I}$ be the product of the projections of $X_{B}$ on the various members of $\mathscr{L}$. Now $X^{\Phi}$ is abelian, so $X_{B}$ and hence also $\tilde{I}$ are abelian. Also $1 \neq X_{B}$ is $H^{\Phi}$-invariant, so by 8.5.1, $X_{B}^{\gamma}$ is transitive for each $\gamma \in \Gamma_{\Phi}$, and hence $\Gamma_{\Phi}$ is the set of orbits of $X_{B}$ on $\Phi$, and $X_{B}^{\gamma}$ is regular. Thus as $I$ centralizes $X_{B}^{\gamma}, X_{B}^{\gamma}=I^{\gamma}$, so $I$ is contained in the product $X_{0}$ of the projections of $X_{B}$ on the $X_{\gamma}$, and $X_{0} \leq X$. Similarly $\tilde{I}$ acts on $\Gamma_{\Phi}$ and

$$
\tilde{I}_{\Gamma_{\Phi}} \leq X_{0} \cap B^{\Phi}=X_{B} .
$$

Thus $[I, \tilde{I}] \leq \tilde{I}_{\Gamma_{\Phi}} \leq X_{B}$, so we have quadratic action of $I$ on $\tilde{I}$. It follows that either (1) holds, or $p=2$ and all orbits of $I$ on $\mathscr{L}$ are of length 2, and we may assume the latter. Therefore $\left|\tilde{I}: X_{B}\right|=\left|X_{B}\right|$. But $\left[H_{*}, \tilde{I}\right] \leq \tilde{I}_{\Gamma_{\Phi}} \leq X_{B}$, so as $H_{*}$ is nontrivial on $\mathscr{L}$ by 8.9.6, $\left|\tilde{I}: X_{B}\right|<\left|X^{B}\right|$, a contradiction. This completes the proof of (1).

Finally suppose (3) fails, so that $\tilde{I} \neq X_{B}$, and hence $\tilde{I} \not \leq X^{\Phi}$. From the previous paragraph, $\tilde{I}_{\Gamma_{\Phi}} \leq X_{B}$, so $\tilde{I} \neq \tilde{I}_{\Gamma_{\Phi}}$. On the other hand $X^{\Phi}$ acts on $X_{B}$ and hence on $\tilde{I}$, so $\left[X^{\Phi}, \tilde{I}\right] \leq \tilde{I}_{\Gamma_{\Phi}} \leq X_{B}$. Therefore $X^{\Phi}$ acts quadratically on $\tilde{I}$, and we obtain a contradiction as in the previous paragraph.

8.12. Assume 8.6.2 holds, choose $W$ as in 8.8 .8 and set $\Phi:=\Phi_{W}$ and $B:=B_{W}$. Then:

(1) $(X \cap W)^{\Phi} \leq B^{\Phi}$.

(2) $(X \cap W)^{\Phi}$ contains its projection on each member of $\mathscr{L}$.

Proof. Part (1) follows from 8.10.2, 8.9.5, and 4.2 in [8].

By 8.10.1, $X \cap W=X(\alpha)$ for a subset $\alpha$ of $\{1, \ldots, s\}$, so by 8.6.2, $X \cap W=$ $E(X \cap W)$. Let $\tilde{X}$ be the product of the projections of $(X \cap W)^{\Phi}$ on the various members of $\mathscr{L}$. Claim $\tilde{X} \neq B^{\Phi}$. Suppose otherwise. Then for $P$ a component of $(X \cap W)^{\Phi}, P$ projects on a component $Q$ of $B^{\Phi}$. Now by 8.9.5, we can identify $\Phi$ with a product

$$
\Phi=\prod_{Q \in \mathscr{L}} \Phi_{Q},
$$

where the $\Phi_{Q}$ are $m$-sets. There exists $x \in Q$ acting without fixed points on $\Phi_{Q}$. Let $y \in P$ project on $x$. Then $y$ has no fixed points on $\Phi$, contrary to the proof of 8.10, which showed $P^{\#} \subseteq \mathcal{R}(\Phi)$. This establishes the claim.

By 8.9.1, $B \cap U$ is maximal in $\mathcal{W}_{0}(<W)$, so by 3.7.3, $H^{\Phi}(B \cap U)^{\Phi}$ is maximal in $B^{\Phi} H^{\Phi}$. Therefore as $\tilde{X}$ is normalized by $H^{\Phi}(B \cap U)^{\Phi}$, it follows from the claim that $\tilde{X} \leq(B \cap U)^{\Phi}$. Thus $P \unlhd \tilde{X}$, so $P$ is one of the components of $\tilde{X}$, and then $(X \cap W)^{\Phi}=\tilde{X}$, completing the proof of the lemma. 
Theorem 8.13. $J \cap D(\Gamma)=1$.

Proof. Assume otherwise. Then Hypothesis 8.4 is satisfied. Adopt Notation 8.7, pick $W$ as in 8.8.8, and set $Y:=Y_{W}, \Phi:=\Phi_{W}$, and $B:=B_{W}$. By 8.9.5, $Y^{\Phi}$ preserves a regular $(m, r)$-product structure on $\Phi$ for some $m \geq 5$ and $r \geq 2$, so we can make an identification

$$
\Phi=\prod_{j=1}^{r} \Phi_{j}
$$

where $\Phi_{j}$ is an $m$-set. If 8.6.1 holds, define $X_{B}$ as in 8.11.2, while if 8.6.2 holds, then let $X_{B}=(X \cap W)^{\Phi}$. Then by 8.11 and $8.12, X_{B} \leq B^{\Phi}$, and $X_{B}$ is the product of its projections on the various members of $\mathscr{L}$.

For $1 \leq j \leq r$, let $\Gamma_{j}$ be the set of orbits of $X_{B}$ on $\Phi_{j}$. Let $\mathcal{A}=\Gamma_{1} \times \cdots \times \Gamma_{r}$ and for $\alpha=\left(\alpha_{1}, \ldots, \alpha_{r}\right) \in \mathcal{A}$, define $\gamma_{\alpha}:=\alpha_{1} \times \cdots \times \alpha_{r} \subseteq \Phi$. Then as $X_{B}$ is the product of its projections:

(1) $\left\{\gamma_{\alpha}: \alpha \in \mathcal{A}\right\}$ is the set $\Gamma_{\Phi}^{\prime}$ of orbits of $X_{B}$ on $\Phi$.

However as $1 \neq X_{B}$ is $H$-invariant, and for each $\gamma \in \Gamma_{\Phi}, H^{\gamma}$ is primitive by 8.1.3, it follows that $X_{B}^{\gamma}$ is transitive, so:

(2) $\Gamma_{\Phi}=\Gamma_{\Phi}^{\prime}$.

Pick $\alpha=\left(\alpha_{1}, \ldots, \alpha_{r}\right) \in \mathcal{A}$. By 8.9.6, $H_{*}$ acts nontrivially on $\mathscr{L}$. Thus identifying $\mathscr{L}$ with $\{1, \ldots, r\}$, there exists $h \in H_{*}$ with $1 h=j \neq 1$. As $|\Gamma|>1, \alpha_{j} \neq \Phi_{j}$, so there exits $\delta_{j} \in \Gamma_{j}-\left\{\alpha_{j}\right\}$. Pick a $\beta \in \mathcal{A}$ with $\beta_{1}=\alpha_{1}$ and $\beta_{j}=\delta_{j}$. As $\Gamma_{\Phi}$ is the set of orbits of $H_{*}$ on $\Phi, H_{*}$ acts on each member of $\Gamma_{\Phi}$, so in particular $\gamma_{\alpha} h=\gamma_{\alpha}$. Hence $\alpha_{1} h=\alpha_{j}$. Similarly $\beta_{1} h=\beta_{j}$. But by construction, $\alpha_{1}=\beta_{1}$ while $\alpha_{j} \neq \beta_{j}$, a contradiction. This completes the proof of the theorem.

\section{The case $H_{*}$ simple}

In this section we continue to assume Hypothesis 6.1, and adopt the notation established there. By 8.1.1, $\mathcal{P}^{\prime}(H)=\mathcal{P}_{1}(H)$. Let $\Gamma$ be the set of orbits of $H_{*}$ on $\Omega$. Then by 8.1.2, $\Gamma$ is the greatest member of $\mathcal{P}^{\prime}(H)$. Set $D:=D(\Gamma)$ and let $\mathscr{L}$ the set of components of $D$. Thus $H$ is transitive on $\mathscr{L}$, the members of $\mathscr{L}$ are simple, and $D$ is the direct product of the members of $\mathscr{L}$.

9.1. The following hold:

(1) $J \cap D=1$.

(2) $H_{*} \cong L$.

(3) $H_{0}=H_{*} \times J$.

(4) $W_{*}=C_{\hat{G}}\left(H_{*}\right) \in W_{0}$. 
Proof. Part (1) is a restatement of Theorem 8.13. Then as $H_{*} \leq D$ by 8.1, it follows that $H_{*} \cap J=1$. Thus $H_{*} \cong H_{*} J / J=H_{0} / J \cong L$, so (2) holds. Further $\left[H_{*}, J\right] \leq D \cap J=1$, so (3) follows.

Let $W_{*}:=C_{\hat{G}}\left(H_{*}\right)$. By (3), $J \leq W_{*}$. On the other hand $H$ acts on $W_{*}$, and $H \cap W_{*} \leq C_{H}\left(H_{*}\right)=C_{H_{0}}\left(H_{*}\right)=J$ by (3), so $W_{*} \in W_{0}$, establishing (4).

\subsection{The following hold:}

(1) $H_{*}$ is not regular on $\gamma \in \Gamma$.

(2) $W_{*}=1$.

(3) $J=1$.

(4) $H$ is almost simple with $F^{*}(H)=H_{*}$.

Proof. Suppose $H_{*}$ is regular on $\gamma \in \Gamma$. Then $C_{\mathrm{Sym}(\gamma)}\left(H_{*}^{\gamma}\right) \cong H_{*} \cong L$ is also regular on $\gamma$. Let $W(\gamma):=C_{A_{\Omega-\gamma}}\left(H_{*}\right)$ and $W_{0}:=\langle W(\gamma): \gamma \in \Gamma\rangle$. Then

$$
W(\gamma) \cong W(\gamma)^{\gamma}=C_{\operatorname{Sym}(\gamma)}\left(H_{*}^{\gamma}\right) \cong L,
$$

and $W_{0}$ is the direct product of the $W(\gamma)$. Further $W_{0} \unlhd W_{*}$, and there is a complement $T$ to $W_{0}$ in $W_{*}$ acting faithfully as $\operatorname{Sym}(\Gamma)$ on $\Gamma$, with $N_{T}(\gamma)$ centralizing $W(\gamma)$; that is $W_{*}$ is the wreath product of $L$ by $\operatorname{Sym}(\Gamma)$.

By 8.1.3, $H^{\gamma}$ is primitive. Thus as $H_{*}^{\gamma} \cong L$ is a minimal normal subgroup of $H^{\gamma}$, either $H^{\gamma}$ is almost simple, or $H^{\gamma}$ is doubled with minimal normal subgroups $H_{*}^{\gamma}$ and $W(\gamma)^{\gamma}$. In the first case as $H_{*}^{\gamma}$ is regular, for $\omega \in \gamma, H_{\omega}^{\gamma}$ is a complement to $H_{*}^{\gamma}$ in $H^{\gamma}$, and maximal in $H^{\gamma}$. This is contrary to the argument in 6.3 in [7]. Thus $H^{\gamma}$ is doubled, and $W(\gamma)^{\gamma}=J^{\gamma}$ by 9.1.3. Thus

$$
\operatorname{Aut}_{J}(W(\gamma))=\operatorname{Inn}(W(\gamma)) .
$$

Claim $J$ is contained in no complement $R$ to $W_{0}$ in $W_{*}$. Assume otherwise and set $m:=|\Gamma|$. Then $R \cong S_{m}$ and $N_{R}(\gamma) \cong S_{m-1}$. But as

$$
\operatorname{Aut}_{J}(W(\gamma))=\operatorname{Inn}(W(\gamma))
$$

it follows that $\operatorname{Inn}(W(\gamma)) \leq \operatorname{Aut}_{R}(W(\gamma))$. Thus as $N_{R}(\gamma)$ is almost simple, $N_{R}(\gamma)$ is faithful on $W(\gamma) \cong L$, and $L \cong F^{*}\left(N_{R}(\gamma)\right) \cong A_{m-1}$. But there is an involution $r \in R-E(R)$ inducing an outer automorphism on $E(R)$, and hence also on $W(\gamma)$. But then as $H_{*}^{\gamma} W(\gamma)^{\gamma}$ is doubled, $r$ also induces an outer automorphism on $H_{*}$, contradicting $r \in W_{*} \leq C_{G}\left(H_{*}\right)$. This completes the proof of the claim.

Next $W_{0}=\left(W_{*}\right)_{\Gamma}$ and $W_{0} \leq D$, so $J \cap W_{0} \leq J \cap D=1$ by 9.1.1. Thus by the claim, $W_{*} \neq W_{0} J=\bar{W}_{0}$, so that $\bar{W}_{0} \in \mathcal{W}_{0}$ and $\bar{W}_{0}<W_{*}$. Therefore by 9.1 .4 
and 3.4, there exists $U \in \mathcal{W}_{0}^{\prime}$ with $W_{*}=W_{0} U$ and $\bar{W}_{0} \cap U=J$. Thus $J \leq U$, and $W_{0} \cap U=W_{0} \cap \bar{W}_{0} \cap U=W_{0} \cap J=1$. Hence $U$ is a complement to $W_{0}$ in $W_{*}$ containing $J$, contrary to the claim. This completes the proof of (1).

Let $\mathcal{O}$ be the set of orbits of $W_{*}$ on $\Omega$. Suppose (2) fails. As $H^{\gamma}$ is primitive, either $W_{*}^{\gamma}=1$ or $W_{\gamma}^{*}$ is transitive, and by (1) it is the former. Thus $W_{*}$ is not transitive on $\Omega$. Therefore $\mathcal{O} \in \mathcal{P}^{\prime}(H)$, so $\mathcal{O} \leq \Gamma$ by 8.1.2. This is impossible as we just saw $W_{*}^{\gamma}=1$. Thus (2) holds. Then as $J \leq W_{*}$ by 9.1.3, (2) implies (3).

As $J=1$ and $H_{*} J / J=F^{*}(H / J)$, (4) holds.

9.3. Let $W \in W_{0}^{\prime}$ and $Y:=W H$. Then:

(1) $H$ is a complement to $W$ in $Y$.

(2) $\operatorname{ker}_{H}(Y)=1$.

Proof. As $W \in W_{0}, H \cap W=J=1$ by 9.2.3. Thus (1) holds. If $1 \neq \operatorname{ker}_{H}(Y)$, then $H_{*} \leq \operatorname{ker}_{H}(Y)$ by 9.2.4. But then

$$
\left[H_{*}, W\right] \leq H \cap W=1
$$

by (1), so $W \leq W_{*}=1$ by 9.2.2, a contradiction. Hence (2) holds.

9.4. Let $W \in W_{0}^{*}$ and $Y:=W H$. Then:

(1) $H$ is maximal in $Y$.

(2) $Y$ is faithful and primitive on $Y / H$.

(3) $W=F^{*}(Y) \cong E_{p^{e}}$ for some prime $p$ and integer $e>1, W$ is regular on $Y / H$, and $Y$ is affine on $Y / H$.

Proof. As $W \in W_{0}^{*}$, part (1) follows from 2.12.1 in [6]. Then (2) follows from (1) and 9.3.2. As $W \unlhd Y$, it follows from (2) that $W$ is transitive on $Y / H$, and then $W$ is regular on $Y / H$ by 9.3.1. As $W$ is regular on $Y / H, Y$ is either affine or complemented, and we may assume the latter. Then $W$ is the direct product of the set $\mathcal{C}$ of the $r$ components of $Y$, and $H$ is faithful and transitive on $\mathcal{C}$, so $H \leq S_{r}$. Let $c$ be the order of a component of $Y$; thus $n=c^{r}$. Pick a prime divisor $p$ of $c$. As $H$ is transitive on $\Omega, n$ divides $|H|$, so $|H|_{p} \geq c_{p}^{r} \geq p^{r}$. But by 3.3 in [4],

$$
\left|S_{r}\right|_{p} \leq p^{(r-1) /(p-1)}<p^{r},
$$

a contradiction.

Theorem 9.5. Assume Hypothesis 5.1. If $H$ is transitive on $\Omega$, then $H$ is primitive on $\Omega$.

Proof. Assume otherwise. Then Hypothesis 6.1 is satisfied. Thus we may appeal to the results in this section. 
Let $W \in W_{0}^{*}$ and $Y:=H W$. By 9.4.3, $W$ is the unique minimal normal subgroup of $Y$ and $W \cong E_{p^{e}}$ for some prime $p$ and integer $e$.

If $Y$ is primitive on $\Omega$, we set $\Phi:=\Omega, K:=H$, and $X:=Y$. Suppose instead that $Y$ is imprimitive on $\Omega$. Then by 8.1.4, there is a greatest member $\Sigma$ of $\mathcal{P}^{\prime}(Y)$, and $\Sigma \leq \Gamma$. As $\Sigma \leq \Gamma, H_{*} \leq G_{\Sigma}$. As $H$ is irreducible on $W$,

$$
W=\left[W, H_{*}\right] \leq G_{\Sigma} .
$$

Thus by maximality of $\Sigma, \Sigma$ is the set of orbits of $W$ on $\Omega$. In this case pick $\Phi \in \Sigma$ and set $K:=N_{H}(\Phi)$ and $X:=W K$. By construction, $H_{*} \leq K \leq H$, so by 9.2.4, $K$ is almost simple with $F^{*}(K)=H_{*}$. As $H$ is transitive on $\Omega$ and preserves $\Sigma$, $K$ is transitive on $\Phi$.

Thus in either case, $K$ is almost simple with $F^{*}(K)=H_{*}, K$ is transitive on $\Phi$, and $|\Phi|$ is a power of $p$. Therefore by 1.5 , either $H_{*}$ is transitive on $\Phi$ or

$$
C_{A}\left(H_{*}\right) \neq 1 \text {. }
$$

The latter contradicts 9.2.2, so the former holds. However as $\Gamma$ is a nontrivial partition of $\Omega$, and the set of orbits of $H_{*}$ on $\Omega, H_{*}$ is not transitive on $\Omega$. Thus $Y$ is imprimitive on $\Omega$ and $\Phi \in \Sigma$. Moreover as $H_{*}$ is transitive on $\Phi, \Phi \in \Gamma$, so $\Sigma=\Gamma$, and hence $W \leq M$. But now by $3.3, G=\left\langle W_{0}^{*}, H\right\rangle \leq M(\Gamma)$, contrary to Hypothesis 5.1.

\section{The intransitive case}

In this section we assume:

Hypothesis 10.1. Hypothesis 5.1 holds and $H$ is intransitive on $\Omega$.

Notation 10.2. Let $\hat{G}:=F^{*}(G) J, I:=\{1, \ldots, k\}$, and $\left\{\Omega_{i}: i \in I\right\}$ the orbits of $H$ on $\Omega$. For $\gamma \subseteq I$, set $\gamma^{\prime}:=I-\gamma$,

$$
\Omega_{\gamma}:=\bigcup_{i \in \gamma} \Omega_{i}, \quad X_{\gamma}:=\hat{G}_{\Omega-\Omega_{\gamma}}, \quad T_{\gamma}:=S_{\Omega-\Omega_{\gamma}}, \quad G_{\gamma}:=\prod_{i \in \gamma} X_{i},
$$

and

$$
S_{I}:=\prod_{i \in I} T_{i}
$$

Recall the definition of $H_{*}$ from 2.11, and set $J_{*}:=J \cap H_{*}$. Set

$$
I_{*}:=\left\{i \in I: \Omega_{i} \subseteq \operatorname{Mov}\left(H_{*}\right)\right\} .
$$

Observe $G_{I}=X_{1} \times \cdots \times X_{k}$, and let $\pi_{i}: G_{I} \rightarrow X_{i}$ be the $i$ th projection map. As $H$ acts on each $\Omega_{i}$, and $\left|S_{I}: G_{I}\right|$ is of exponent $2, O^{2}(H) \leq G_{I}$. 
For $Y \in d_{G}(H)$, set $\bar{Y}:=Y J$. Let $\delta$ be the set of subsets $\gamma$ of $I$ such that $I_{*} \nsubseteq \gamma$.

10.3. The following hold:

(1) $H_{*} \leq G_{I_{*}}$.

(2) For each $i \in I_{*}$, set $H_{i}:=H_{*} \pi_{i}$ and $J_{i}:=J_{*} \pi_{i}$. Then

$$
H_{i} / J_{i} \cong L,
$$

so $\left|\Omega_{i}\right| \geq 5$.

(3) For $\gamma \in \mathcal{S}, \bar{X}_{\gamma}$ and $\bar{G}_{\gamma}$ are in $\mathcal{W}_{0}$.

(4) Assume $\alpha_{j} \in 8$ for $j=1,2$ such that $\alpha_{2} \nsubseteq \alpha_{1},\left|\alpha_{2}\right|>1$, and $\left|\Omega_{\alpha_{2}}\right|>2$. Then $\bar{X}_{\alpha_{1}} \neq \bar{X}_{\alpha_{2}}$ and $\bar{X}_{\alpha_{2}} \in \mathcal{W}_{0}^{\prime}$.

(5) For $i \in I_{*}, X_{i} \not \leq J$.

(6) Suppose $\alpha_{j} \in \mathcal{S}, j=1,2$, and there exists $i \in \alpha_{2} \cap I_{*}-\alpha_{1}$. Then $\bar{G}_{\alpha_{1}} \neq \bar{G}_{\alpha_{2}}$.

Proof. If $H_{*}$ is nontrivial on $\Omega_{i}$, then as $H$ is transitive on $\Omega_{i}$ and $H_{*} \unlhd H$, we have $i \in I_{*}$. Hence $H_{*} \leq S_{I_{*}}$. Then as $H_{*}=O^{2}\left(H_{*}\right)$, (1) follows from a remark in 10.2 .

For $i \in I_{*}, \Omega_{i} \subseteq \operatorname{Mov}\left(H_{*}\right)$, so $H_{i} \neq 1$. If $H_{i} \leq J_{i}$, then for $x \in H_{*}-J_{*}$, there is $y \in J_{*}$ with $x \pi_{i}=y \pi_{i}$. Thus $x y^{-1} \in \operatorname{ker}\left(\pi_{i}\right)$, so

$$
\operatorname{ker}\left(\pi_{i}\right) \cap H_{*} \not J_{*} .
$$

Hence as $\operatorname{ker}\left(\pi_{i}\right)=G_{i^{\prime}}$ is $H$-invariant, we get $H_{*} \leq \operatorname{ker}\left(\pi_{i}\right)$ by 2.11 , contradicting $H_{i} \neq 1$. Therefore $H_{i} \not \leq J_{i}$, so the induced map $\zeta: H_{*} / J_{*} \rightarrow H_{i} / J_{i}$ defined by $\zeta: h J_{*} \mapsto\left(h \pi_{i}\right) J_{i}$ is nontrivial. Then as $H_{*} / J_{*} \cong L$ is simple, (2) holds.

Pick $\gamma$ as in (3) and let $Y \in\left\{G_{\gamma}, X_{\gamma}\right\}$. For $i \in \gamma, \Omega_{i}$ is $H$-invariant, so $Y$ is $H$-invariant. Thus if $\bar{Y} \notin \mathcal{W}_{0}$, then we get $H_{*} \leq Y$ by 2.12. But as $\gamma \in \delta$, there is $i \in I_{*}-\gamma$, and by (2), $H_{*} \pi_{i} \neq 1$, a contradiction. Therefore (3) holds.

Assume the hypothesis of (4). Then $X_{\alpha_{1}}$ acts on $\Omega_{i}$ for $i \in \alpha_{2}-\alpha_{1}$, but as $\left|\Omega_{\alpha_{2}}\right|>2, X_{\alpha_{2}}$ is transitive on $\Omega_{\alpha_{2}}$, and then as $\left|\alpha_{2}\right|>1, X_{\alpha_{2}}$ does not act on $\Omega_{i}$. Thus as $J$ acts on $\Omega_{i}, \bar{X}_{\alpha_{1}}=X_{\alpha_{1}} J \neq X_{\alpha_{2}} J=\bar{X}_{\alpha_{2}}$, and $\bar{X}_{\alpha_{2}} \neq J$. Therefore (4) holds.

Suppose $i \in I_{*}$ with $X_{i} \leq J$, and let $\gamma=I_{*}-\{i\}$. Then by (1),

$$
H_{*} \leq G_{I_{*}}=G_{\gamma} X_{i} \leq G_{\gamma} J=\bar{G}_{\gamma},
$$

contrary to (3). Thus (5) holds. 
Assume the hypothesis of (6) and $\bar{G}_{\alpha_{1}}=\bar{G}_{\alpha_{2}}$. By hypothesis there exists an $i \in I_{*} \cap \alpha_{2}-\alpha_{1}$. Therefore $G_{i} \leq \bar{G}_{\alpha_{2}}=\bar{G}_{\alpha_{1}}=G_{\alpha_{1}} J \leq G_{i^{\prime}} J$. But by (1),

$$
H_{*} \leq G_{I_{*}} \leq G_{i^{\prime}} G_{i} \leq G_{i^{\prime}} J=\bar{G}_{i^{\prime}},
$$

whereas $\bar{G}_{i^{\prime}} \in \mathcal{W}_{0}$ by (3).

10.4. Suppose $\gamma \in 8$ and $\alpha_{j} \subseteq \gamma$ for $j=1,2$, such that $\left|\Omega_{\alpha_{1}}\right|=\left|\Omega_{\alpha_{2}}\right|=m>0$ and $\alpha_{1} \cap \alpha_{2}=\varnothing$. Then $m=1,|\gamma| \leq 3$ with $\left|\Omega_{i}\right|=1$ for $i \in \gamma-\left(\alpha_{1} \cup \alpha_{2}\right)$, and $\hat{G}=A$.

Proof. By 10.3.3, $\bar{X}_{\gamma} \in \mathcal{W}_{0}$. Let $Y:=N_{X_{\gamma}}\left(\left\{\Omega_{\alpha_{1}}, \Omega_{\alpha_{2}}\right\}\right)$ and $X:=N_{Y}\left(\Omega_{\alpha_{1}}\right)$. Then $X$ and $Y$ are $H$-invariant, so $\bar{X}, \bar{Y} \in \mathcal{W}_{0}$ by 2.7. Also $|Y: X| \leq 2$, and as $J$ acts on $\Omega_{\alpha_{1}}$, if $Y \neq X$, then $\bar{Y} \neq \bar{X}$. Therefore by 3.5.2, $Y=X$. The lemma follows.

10.5. $k \leq 3$.

Proof. Assume $k>3$. Then we may choose notation so that some member of $I_{*}$ is not contained in $I_{0}=\{1,2,3\}$, and hence $I_{0} \in \mathcal{S}$. Let $I_{1}=\left\{i \in I_{0}:\left|\Omega_{i}\right|=1\right\}$, and let $I_{2}$ be the set of 2-subsets of $I_{0}$. Set $X:=X_{I_{0}}$.

Assume $\left|\Omega_{\alpha}\right|>2$ for each $\alpha \in I_{2}$. Then by 10.3.4 applied to $\alpha, i_{\alpha}$ in the role of $\alpha_{2}, \alpha_{1}$, with $i_{\alpha} \in \alpha, \bar{X}_{\alpha} \in \mathcal{W}_{0}^{\prime}$ and $\bar{X}_{\alpha} \neq \bar{X}_{i_{\alpha}}$. Similarly by $10.3 .4, \bar{X} \in W_{0}$ and $\bar{X}_{\alpha} \neq \bar{X}$. Observe that for distinct $\alpha$ and $\beta \in I_{2}$,

$$
X=\left\langle X_{\alpha}, X_{\beta}\right\rangle \quad \text { and } \quad X_{\alpha} \cap X_{\beta}=X_{\alpha \cap \beta} .
$$

Applying 3.4 to the connected component $\mathcal{C}$ of $\Lambda^{\prime}$ containing $\bar{X}$, it follows that $\bar{X}=L_{\mathcal{B}}$ for some $\mathcal{B} \subseteq \mathcal{C}^{*}, \bar{X}_{\alpha}=L_{\nu(\alpha)}$ for some $\nu(\alpha) \subseteq \mathscr{B}$, and

$$
\bar{X}_{\alpha \cap \beta}=L_{v(\alpha) \cap v(\beta)} .
$$

Then by 3.4 and 3.7, $v(\alpha) \cup v(\beta)=\mathscr{B}$ and $v(\alpha \cap \beta)=v(\alpha) \cap v(\beta)$. But now

$$
\mathscr{B}=v(\{1,2\}) \cup v(\{1,3\}) \cup v(\{2,3\}),
$$

so as $X_{i, j} \cap X_{i, l}=X_{i}$, it follows from 3.4 that $X=Y$, where $Y=\left\langle X_{1}, X_{2}, X_{3}\right\rangle$. As $Y$ acts on $\Omega_{i}$ for each $1 \leq i \leq 3$, but $X$ does not, this is a contradiction.

Therefore $\left|\Omega_{\alpha}\right|=2$ for some $\alpha \in I_{2}$. We may take $\alpha=\{1,2\}$, so $\left|\Omega_{i}\right|=1$ for $i \in\{1,2\}$. Therefore by $10.4,\left|\Omega_{j}\right|=1$ for each $j \in I_{0}$, and $\hat{G}=A$. Since we could have chosen $I_{0}$ to be any 3 -subset of $I$ not contained in $I_{*}$, and since $\left|\Omega_{i}\right| \geq 5$ for each $i \in I_{*}$ by 10.3 .2 , it follows that $\left|I_{*}\right|=1$ and $\left|\Omega_{i}\right|=1$ for each $i \in I_{*}^{\prime}$. Finally if $k>4$, we obtain a contradiction from 10.4 by choosing $\alpha_{j}=\{j\}$ for $j=1,2$ and $\gamma=I_{*}^{\prime}$ in that lemma. Thus we have shown that $k=4$, and we may choose notation so that $I_{*}=\{4\}$ and $\left|\Omega_{i}\right|=1$ for $1 \leq i \leq 3$. 
Now as $\hat{G}=A, X \cong Z_{3}$. Further all orbits of $H$ on $\Omega_{1,2,3}$ are of length 1, so $H$ centralizes $X$ and $J \cap X=1$. Therefore $J \neq \bar{X}=N_{\bar{X}}(H)$, contrary to 3.5.1. This completes the proof of the lemma.

10.6. Assume $\{1,2\} \in \mathcal{S}$ and $\bar{X}_{2} \in W_{0}^{\prime}$. Then $\bar{X}_{2}=\bar{G}_{1,2}$, so $X_{1} \leq J$.

Proof. Assume otherwise. By 10.3.3, $\bar{X}_{1,2}, \bar{X}_{j}, j=1,2$, and $\bar{G}_{1,2}$ are in $\mathcal{W}_{0}$. By hypothesis, we have $\bar{X}_{2} \in W_{0}^{\prime}$ and $\bar{X}_{2} \neq \bar{G}_{1,2}$, so also $\bar{X}_{1} \in W_{0}^{\prime}$. Let $m_{j}=\left|\Omega_{j}\right|$. As $\bar{X}_{j} \in \mathcal{W}_{0}^{\prime}, m_{j}>2$ by 3.5.3. By 10.4, $m_{1} \neq m_{2}$, so we may assume $m_{2}>m_{1}$.

We apply 3.4 and 3.7 to $X_{1,2}$ in the role of $Y, X_{1,2} H$ in the role of $X$, and $\mathcal{C}$ the connected component of $\Lambda^{\prime}$ containing $\bar{X}_{1,2}$. In particular $\bar{X}_{1,2}=L_{\mathscr{A}}$ for some $\mathcal{A} \subseteq \mathcal{C}^{*}, \bar{X}_{i}=L_{\alpha_{i}}$ for some $\alpha_{i} \subseteq \mathcal{A}$, and $\bar{G}_{1,2}=L_{\gamma}$, where $\gamma=\alpha_{1} \cup \alpha_{2} \subset \mathcal{A}$ as $\bar{G}_{1,2} \neq \bar{X}_{1,2}$. Pick $a \in \mathcal{A}-\gamma$, and set

$$
\beta:=\alpha_{2} \cup\{a\}, \quad U:=L_{\beta}, \quad \text { and } \quad Z:=X_{1,2} \cap U .
$$

By 3.7, $U=\bar{Z}$, and as $\beta \subset \mathcal{A}, Z \neq X_{1,2}$. As $a \notin \gamma$, it follows that $Z \not G_{1,2}$, so $Z_{H}$ is transitive on $\Omega_{1,2}$. As $m_{2}>2, X_{2}$ contains a 3 -cycle, so as $Z \neq X_{1,2}$ and $Z H$ is transitive on $\Omega_{1,2}$, it follows from 3.2 in [8] that $Z$ is imprimitive on $\Omega_{1,2}$, and $\Omega_{2}$ is contained in a block of each nontrivial $Z$-invariant partition of $\Omega_{1,2}$. As $m_{2}>m_{1}=\left|\Omega_{1,2}-\Omega_{2}\right|$, this is a contradiction.

10.7. Assume $k=3$. Then:

(1) $I_{*}^{\prime} \neq \varnothing$, and

(2) for some $i \in I_{*}^{\prime}, X_{i} \leq J$.

Proof. Assume otherwise. We may choose $I_{0}=\{1,2\} \in 8$. Thus for $i \in I_{0}$ we have $\bar{X}_{i} \in \mathcal{W}_{0}$ by 10.3.3. If also $i \in I_{*}$, then $\bar{X}_{i} \in \mathcal{W}_{0}^{\prime}$ by 10.3 .5 . Therefore by 10.6, there exists $j \in I_{0} \cap I_{*}^{\prime}$ such that $X_{j} \leq J$, completing the proof.

Theorem 10.8. Assume $k=3$ and choose notation so that $I_{*}=\{3\}$ or $\{2,3\}$. If $I_{*}=\{3\}$, assume $X_{2} \neq 1$. Then:

(1) $\left|\Omega_{1}\right| \neq\left|\Omega_{2}\right|$, and

(2) $N_{X_{1,2}}\left(\Omega_{1}\right) \leq J$.

We prove Theorem 10.8 in a series of reductions, so assume the hypotheses of the theorem. For $1 \leq i \leq 3$, set $m_{i}:=\left|\Omega_{i}\right|$, and set $M:=N_{X_{1,2}}\left(\Omega_{1}\right)$.

If $I_{*}=\{3\}$, then $X_{2} \neq 1$ by hypothesis, while if $I_{*}=\{2,3\}$, this follows from 10.3.2. Thus

10.9. $X_{2} \neq 1$. 
By 10.9, $m_{2}>1$, so 10.8.1 follows from 10.4. By 10.8.1 and 3.7.1 in [8]:

10.10. $M$ is a maximal subgroup of $X_{1,2}$.

In the remainder of the proof of Theorem 10.8, assume $M \not \subset J$.

10.11. The following hold:

(1) $\bar{M}, \bar{X}_{1,2} \in W_{0}^{\prime}$ with $\bar{M}<\bar{X}_{1,2}$.

(2) There exists a unique $U \in W_{0}^{*}$ contained in $\bar{X}_{1,2}$ but not in $\bar{M}$. Set

$$
U_{X}:=U \cap X_{1,2} \text {. }
$$

(3) $H U_{X}$ is transitive on $\Omega_{1,2}$.

Proof. By 10.3.3, we have $\bar{X}_{1,2} \in \mathcal{W}_{0}$. Hence as $M \in d_{X_{1,2}}(H), \bar{M} \in \mathcal{W}_{0}$ by 2.7, so as $M \not \subset J, \bar{M} \in \mathcal{W}_{0}^{\prime}$. As $M$ acts on $\Omega_{1}$ but $X_{1,2}$ does not, $\bar{M}<\bar{X}_{1,2}$ by an argument in the proof of 10.3.4, so (1) holds.

As $M$ is maximal in $X_{1,2}, \bar{M}$ is maximal in $W_{0}\left(<\bar{X}_{1,2}\right)$ by 3.7 , so (2) follows from 3.4. By maximality of $M, X_{1,2}=\left\langle U_{X}, M\right\rangle$, and in particular $U_{X} \not \leq M$. Then as $H$ is transitive on $\Omega_{i}$, (3) follows.

Let $\mathcal{C}$ be the connected component of $\Lambda^{\prime}$ containing $\bar{X}_{1,2}$ and $\mathcal{C}^{*}:=\mathcal{C} \cap W_{0}^{*}$. By 3.8 some $V \in \mathcal{C}^{*}$ does not act on $\Omega_{1,2}$. Let

$$
U_{X} \leq Y \in d_{X_{1,2}}(H)
$$

with $\bar{Y} \neq \bar{X}_{1,2}$, and set $Z_{Y}:=\langle Y, V\rangle$ and $B_{Y}:=Z_{Y} H$.

10.12. The following hold:

(1) $Z_{Y} \in \mathcal{C}, \bar{Y} \cap V=J$, and $\bar{X}_{1,2} \cap Z_{Y}=\bar{Y}$.

(2) $B_{Y}$ is transitive on $\Omega$.

(3) $A \not \leq B_{Y}$.

Proof. Part (1) follows from 3.4. As $U H$ is transitive on $\Omega_{1,2}$ and $\Omega_{3}$, and as $V$ does not act on $\Omega_{1,2}$, (2) holds. As $Z_{Y} \in \mathcal{C}$, (3) holds.

By 10.7, $X_{i} \leq J$ for $i=1,2$. Choose such an $i \in\{1,2\}$.

10.13. Assume $X_{i} \neq 1$.

(1) $\Delta_{Y}:=\Omega_{1}^{Y}$ is a $U H$-partition of $\Omega_{1,2}, Y H$ is 2-transitive on $\Delta_{Y}, Y$ is transitive on $\Omega_{1,2}$, and $K_{+}\left(\Delta_{Y}\right) \leq Y$.

(2) We may assume $i=1$.

(3) $m_{1}<m_{2}$. 
Proof. Set $j=3-i$. As $X_{1,2} \not \leq Y, 3.2$ in [8] says that there is a $Y H$-partition $\Delta$ on $\Omega_{1,2}$ such that $\Omega_{i} \subseteq \delta \in \Delta$ and $K_{+}(\Delta) \leq Y$. As $H$ is transitive on $\Omega_{1,2}-\Omega_{i}$, it follows that $\Omega_{i}=\delta$ and $Y H$ is 2-transitive on $\Delta$. In particular $Y$ is transitive on $\Delta$ and hence also on $\Omega_{1,2}$, and $m_{i} \leq m_{j}$. This proves (1), and $m_{i}<m_{j}$ by 10.8.1.

By symmetry between $i$ and $j, X_{j} \not \leq J$. Thus by 10.7.2, $i \in I_{*}^{\prime}$. Thus by the hypothesis of Theorem 10.8, either $i=1$ or $I_{*}=\{3\}$. Moreover in the latter case, interchanging 1 and 2 if necessary, we may assume $i=1$. Thus $m_{1}<m_{2}$.

10.14. We may assume $m_{1}<m_{2}$.

Proof. If $X_{i} \neq 1$, this follows from 10.13, so assume otherwise. Thus $m_{i} \leq 2$. If $I_{*}=\{2,3\}$, then $m_{2} \geq 5$ by 10.2 .3 , so $i=1$ and the lemma holds. If $I_{*}=\{3\}$, interchanging 1 and 2 if necessary, and appealing to 10.8.1, the lemma holds.

10.15. $V$ acts on $\Omega_{2}$.

Proof. Set $W=\langle M, V\rangle$ and assume $V$ does not act on $\Omega_{2}$. Then either $W$ is transitive on $V$ or $W H$ has orbits $\left\{\Omega_{2}, \Omega_{1,3}\right\}$ or $\left\{\Omega_{1}, \Omega_{2,3}\right\}$, so we may assume $W$ is transitive on $\Psi=\Omega$ or $\Omega_{2,3}$. As $H_{*} \leq X_{2,3}$, we have $X_{2,3} \not \leq W$. On the other hand $1 \neq X_{2} \leq M \leq W$, so by 3.2 in [8] there is a nontrivial $W H$-invariant partition $\Sigma$ of $\Psi$ such that $\Omega_{2} \subseteq \sigma \in \Sigma$ and $K_{+}(\Sigma) \leq W$. As $H$ is transitive on $\Omega_{1}$ and $\Omega_{3}$, and as $m_{1}<m_{2}$ by 10.14 , it follows that either $\sigma=\Omega_{2}$ or $\Psi=\Omega$ and $\sigma=\Omega_{1,2}$. In the latter case, $X_{1,2} \leq K_{+}(\Sigma) \leq W$, so $U \leq W$, contrary to 3.4. Thus $\Omega_{2}=\sigma$.

Next for $\omega \in \Omega_{1}$, we have $\omega \in \alpha \in \Sigma$ and $\omega K_{+}(\Sigma)=\alpha$ is of order $m_{2}>m_{1}$, so $K_{+}(\Sigma)$ does not act on $\Omega_{1}$. Thus $K_{+}(\Sigma) \not \leq \bar{M}$, so $V \leq K_{+}(\Sigma) J$. But then $V$ acts on $\Omega_{2}$, contrary to assumption.

10.16. $\Omega_{1,2}^{B_{Y}}$ is not a partition of $\Omega$.

Proof. Assume otherwise. First, $Y$ acts on $\Omega_{1,2}$, and by $10.15, V$ acts on $\Omega_{2}$, so $V$ also acts on $\Omega_{1,2}$. But then $B_{Y}=\langle V, Y, H\rangle$ acts on $\Omega_{1,2}$, contrary to 10.12.2.

10.17. $X_{1}=1$.

Proof. Assume otherwise. Applying 10.13 to $U$ in the role of $Y$, we obtain the $U H$-partition $\Delta=\Delta_{U}=\Omega_{1}^{U}$. By 10.13.1, we have $K_{+}(\Delta) \leq U \leq Y$, so as $\Delta$ is the unique $K_{+}(\Delta)$-invariant partition of $\Omega_{1,2}$ with blocks of size $m_{1}$, we conclude that $\Delta=\Delta_{Y}$ is independent of $Y$. In particular $N_{X_{1, \underline{2}}}(\Delta)$ is the unique maximal overgroup of $U_{X}$ in $X_{1,2}$, so it follows from 3.4 that $M \in W_{0}^{*}$. Then applying 3.7 with $X_{1,2}, X_{1,2} H$ in the roles of $Y, X$, we conclude that $U_{X}$ is a maximal $H$-invariant subgroup of $X_{1,2}$. Thus $U_{X}=N_{X_{1,2}}(\Delta)$ and $Y=U_{X}$. Set $Z=Z_{Y}$ and $B=B_{Y}$. 
By $10.12 .2, B$ is transitive on $\Omega$, and as $H_{*} \not \leq Z$, we have $A \not \leq B$. Therefore by 3.2 in [8], there is a $B$-partition $\Gamma$ of $\Omega$ with $\Omega_{1} \subseteq \gamma \in \Gamma$ and $K_{+}(\Gamma) \leq Z$. As $H$ has orbits $\Omega_{k}, 1 \leq k \leq 3$, it follows that $\gamma=\Omega_{1}, \Omega_{1,2}$, or $\Omega_{1,3}$. As $\bar{Z} \cap \bar{M}=J$, $M \not \leq Z$, so as $K_{+}(\Gamma) \leq Z, \gamma \neq \Omega_{1,2}$.

Suppose $\gamma=\Omega_{1,3}$ and let $u \in U$ with $\Omega_{1} u \neq \Omega_{1}$. Then $\gamma u \neq \gamma$ so $\gamma u \subseteq \Omega_{2}$, and then as $X_{1,3} \leq K_{+}(\Gamma) \leq Z$, we have $X_{1,3}^{u} \leq Z \cap X_{2}=Y$, impossible as $X_{1,3}^{u}$ does not preserve $\Delta$. Therefore $\gamma=\Omega_{1}$, so $\Gamma=\Omega_{1}^{Z}$. In particular $\Delta \subseteq \Gamma$.

Next $Y^{\Delta}=\operatorname{Sym}(\Delta)$ and $H$ is transitive on $\Gamma-\Delta$, so if $B$ is imprimitive on $\Gamma$, then $\Delta^{B}$ is a partition of $\Gamma$. But then $\Omega_{1,2}^{B}$ is a partition of $\Omega$, contrary to 10.16 .

Therefore $B$ is primitive on $\Gamma$. Let $K$ be the kernel of the action of $B$ on $\Gamma$. Then $K / K_{+}(\Gamma)$ is solvable and $K_{+}(\Gamma) \leq Z$, so $\bar{K} \in \mathcal{W}_{0}$ by 2.8 . Then $K \leq Z$ by 3.4. Also as $Y^{\Delta}=\operatorname{Sym}(\Delta), 3.2$ in [8] says $\operatorname{Sym}(\Gamma)=B^{\Gamma}$, contrary to 2.15 .

10.18. $m_{1} \leq 2$.

Proof. This follows from 10.17 and 10.9.

10.19. If $Y$ is transitive on $\Omega_{1,2}$, then $B_{Y}$ is imprimitive on $\Omega$.

Proof. Assume otherwise. By 1.7.3, $B:=B_{Y}$ is 2-transitive on $\Omega$, so by $2.15, B$ is affine and $D=F^{*}(B) \leq Z$. Thus $\bar{D} \in \mathcal{W}_{0}$ by 2.7 . We apply 3.7 to $D, B$ in the roles of $Y, X$, and adopt the notation of that lemma. As $D \not \leq J$, it follows that $\mathcal{A} \neq \varnothing$ and $D J=\bar{D}=L_{\mathcal{A}}$. Let $a \in \mathcal{A}$; then $1 \neq D_{a}=L_{a} \cap D$ is $J D$-invariant as $D$ is abelian, and by 3.7, $D_{a}$ is $L_{\mathscr{B}}$-invariant, so

$$
D_{a} \unlhd\left\langle L_{\mathcal{A}}, L_{\mathcal{B}}, H\right\rangle=B
$$

using 3.4. Therefore as $B$ is irreducible on $D, D=D_{a}$, so $\mathcal{A}=\{a\}$. As $Y$ acts on $\Omega_{2}, L_{a} \not \bar{Y}$, so $L_{a}=V$. Thus $B=\langle Y, V, H\rangle=\bar{Y} D$. But now 1.7.4 supplies a contradiction.

10.20. $m_{1}=2$.

Proof. Assume otherwise and let $B:=B_{Y}$. Then $m_{1}=1$ by 10.18 . By 10.11.3, $H Y$ is transitive on $\Omega_{1,2}$, so as $H$ is transitive on $\Omega_{2}, H Y$ is 2-transitive on $\Omega_{1,2}$. By $10.19, B$ is imprimitive on $\Omega$. Therefore by $1.7 .2, \Gamma=\Omega_{1,2}^{B}$ is a partition of $\Omega$, contradicting 10.16 .

10.21. The following hold:

(1) There exists a nontrivial $Y H$-partition $\Delta$ on $\Omega_{1,2}$.

(2) Either $\Omega_{1} \in \Delta$ or $H_{\Omega_{1}}$ has two orbits $\theta$, $\theta$ h of length $m_{2} / 2$ on $\Omega_{2}$, for $h \in$ $H-H_{\Omega_{1}}$, and $\Delta=\{\Xi, \Xi h\}$, where $\Xi=\{\omega\} \cup \theta$ for some $\omega \in \Omega_{1}$. 
(3) We may choose $Y=N_{X_{1,2}}(\Delta)$, so $Y$ is transitive on $\Omega_{1,2}$.

(4) If $Y=N_{X_{1,2}}(\Delta)$, then there is a nontrivial $B_{Y}$-partition $\Gamma$ of $\Omega$ with $\Delta \subseteq \Gamma$.

Proof. Set $B:=B_{Y}$. Suppose that (1) fails. By 1.6 applied to the action of $Y H$ on $\Omega_{1,2}$, with $\Omega_{1}$ in the role of $\Sigma, Y H$ is 2-transitive on $\Omega_{1,2}$. Then by 1.7.1, $Y$ is transitive on $\Omega_{1,2}$, so by $10.19, B$ is imprimitive on $\Omega$. Then 1.7.2 applied to the action of $B$ on $\Omega, \Omega_{1,2}^{B}$ is a partition of $\Omega$, contrary to 10.16 .

This establishes (1). Then (2) follows from (1) and 1.6. As $H U$ acts on $\Delta$, (3) follows.

Assume $Y=N_{X_{1,2}}(\Delta)$. By (3) and 10.19 there is a nontrivial $B$-partition $\Gamma$ on $\Omega$. Let $\gamma \in \Gamma$ with $\gamma \cap \Omega_{1,2} \neq \varnothing$. By 1.1, either $\Omega_{1,2} \subseteq \gamma$ or $\gamma \subseteq \Omega_{1,2}$. In the former case as $H$ is transitive on $\Omega_{3}, \gamma=\Omega_{1,2}$, contrary to 10.16 . Therefore $\Delta_{1}=\left\{\gamma \in \Gamma: \gamma \subseteq \Omega_{1,2}\right\}$ is a nontrivial $Y H$-partition of $\Omega_{1,2}$, so as $\Delta$ is the unique nontrivial $Y H$-partition of $\Omega_{1,2}, \Delta_{1}=\Delta$. Therefore $\Delta \subseteq \Gamma$, completing the proof of (4).

Choose $\Gamma$ and $\Delta$ as in 10.21 and $Y=N_{X_{1,2}}(\Delta)$. Let $\gamma=\Omega_{1}$ or $\Xi$ in the respective case of 10.21.2; thus $\gamma \in \Gamma$. By construction:

10.22. $\Gamma-\Delta \subseteq \operatorname{Fix}(Y)$ and $Y^{\Delta}=\operatorname{Sym}(\Delta)$.

Set $B:=B_{Y}$ and $Z:=Z_{Y}$. By10.22 we can apply 1.7 to the action of $B^{\Gamma}$ on $\Gamma$. Then if $B^{\Gamma}$ is imprimitive, we conclude from 1.7.2 that $\Delta^{B}$ is a $B$-partition of $\Gamma$. But then $\Omega_{1,2}^{B}$ is a $B$-partition of $\Omega$, contrary to 10.16 .

Therefore $B^{\Gamma}$ is primitive, so it follows from 10.22 and 3.2.1 in [8] that

$$
B^{\Gamma}=\operatorname{Sym}(\Gamma) .
$$

We claim that $B_{\Gamma} \leq Z$. If so, then as $Y^{\Gamma} \neq 1, B_{\Gamma}<Z$, so as $B^{\Gamma}=\operatorname{Sym}(\Gamma)$, 2.15.1 supplies a contradiction. Thus it remains to establish the claim. But $Y$ contains $K_{+}(\Delta)$, so as $B^{\Gamma}$ is transitive, $Z$ contains $K_{+}(\Gamma)$. Then as $B_{\Gamma} / K_{+}(\Gamma)$ is a 2-group, appealing to 2.13 and enlarging $Z$ to $Z B_{\Gamma}$ if necessary, we have $B_{\Gamma} \leq Z$, establishing the claim. This completes the proof of Theorem 10.8 .

10.23. Assume $k=3$. Then:

(1) $\left|I_{*}\right|=1$, so we may choose notation so that $I_{*}=\{1\}$.

(2) Assume $\left|\Omega_{2,3}\right|>2$, and set $M:=N_{X_{2,3}}\left(\Omega_{2}\right)$. Then

$$
M=X_{2,3} \cap J \text { and } \bar{X}_{2,3} \in \mathcal{W}_{0}^{*} .
$$

Proof. By 10.7.1, $\left|I_{*}\right|=1$ or 2 . If $\left|I_{*}\right|=2$, then we may take $I_{*}=\{2,3\}$, so by $10.8, X_{2} \leq J$, contrary to 10.3 .5 . Thus (1) is established and we choose $I_{*}=\{1\}$. 
It remains to prove (2), so we may assume $m:=\left|\Omega_{2,3}\right|>2$. Suppose $X_{i}=1$ for $i=2,3$. Then $m_{i}=\left|\Omega_{i}\right| \leq 2$ for $i=2,3$, so as $m>2$, we may assume $m_{i}=2$ for some $i \in\{2,3\}$. Then as $X_{i}=1, \hat{G}=A$. It follows that either $m=3$ and the subgroup $M$ of (2) is trivial, or $m=4$. In the former case, $Z_{3} \cong X_{2,3}$, so $\bar{X}_{2,3} \in \mathcal{W}_{0}^{*}$ by 2.8 , and hence (2) holds. Thus we may assume the latter case holds. But now $m_{2}=m_{3}=2$, and hence 10.4 supplies a contradiction.

Thus we may assume that $X_{2} \neq 1$. Then, modulo change of notation, the hypotheses of 10.8 are satisfied, and we conclude from that theorem that $M \leq J$, and $m_{2} \neq m_{3}$, so $M$ is maximal in $X_{2,3}$ (cf. 3.7 .1 in [8]). By 10.3.4, $\bar{X}_{2,3} \in W_{0}^{\prime}$, so it follows from the maximality of $M$ in $X_{2,3}$ and 3.7 that

$$
M=X_{2,3} \cap J \quad \text { and } \quad \bar{X}_{2,3} \in \mathcal{W}_{0}^{*} .
$$

This completes the proof of the lemma.

Hypothesis 10.24. $\left|I_{*}\right|=1$.

Notation 10.25. When assuming Hypothesis 10.24, we adopt the following notation: Take $I_{*}:=\{1\}$ and let $X:=X_{1^{\prime}}$ and $\Omega_{X}:=\Omega_{1^{\prime}}$. Observe that Hypothesis 10.24 is always satisfied when $k=3$ by 10.23 .1, so we always adopt Notation 10.24 when $k=3$.

10.26. Assume $k=3$. Then exactly one of the following holds:

(1) $\left|\Omega_{X}\right|>2$ and $\bar{X} \in W_{0}^{*}$.

(2) $\left|\Omega_{X}\right|=2, \hat{G}=A$, and $X=1$.

Proof. If $\left|\Omega_{X}\right|=2$, then (2) holds by 10.4 , so we may assume $\left|\Omega_{X}\right|>2$. Then (1) holds by 10.23.2.

10.27. Assume Hypothesis 10.24 , and suppose $Y_{1} \in \mathcal{d}_{X_{1}}(H)$ with $\bar{Y}_{1} \in \mathcal{W}_{0}$. Then:

(1) For each $Y_{2} \in d_{X}(H), \bar{Y}_{1} Y_{2} \in W_{0}$.

(2) If $\bar{Y}_{2} \leq \bar{Y}_{1}$, then $Y_{2} \leq S_{I}$ and $Y_{2} \leq N_{G}(U \cap X)$ for each $U \in \mathcal{W}$.

(3) Assume $Y_{1} \not \leq J$ and $\bar{Y}_{1}=\bar{X}$. Then $k=2, \bar{X} \in W_{0}^{*}, \hat{G}=A,\left|\Omega_{X}\right|>2$, and $J \cap X=O_{2}(X)$.

Proof. Let $Y_{2} \in \mathcal{d}_{X}(H)$ and set $B:=Y_{1} Y_{2} J \cap X_{1} X$. Then $B=Y_{1} Y_{2} J_{I}$, where $J_{I}:=J \cap X_{1} X \leq S_{I}$.

Suppose (1) fails. Then by $2.12, H_{*} \leq B$. Set $B^{*}=B / Y_{1}$. Then $B^{*}=Y_{2}^{*} J_{I}^{*}$ and $H_{*}$ centralizes $Y_{2}$ and acts on $J_{I}$, so

$$
H_{*}^{*}=\left[H_{*}^{*}, H_{*}^{*}\right] \leq\left[H_{*}^{*}, Y_{2}^{*} J_{I}^{*}\right]=\left[H_{*}^{*}, J_{I}^{*}\right] \leq J_{I}^{*} .
$$

Therefore $H_{*} \leq Y_{1} J_{I} \leq \bar{Y}_{1}$, contradicting $\bar{Y}_{1} \in \mathcal{W}_{0}$. This establishes (1). 
Assume $\bar{Y}_{2} \leq \bar{Y}_{1}$. Then $Y_{2} \leq X_{1} J \leq S_{I}$ as $J \leq S_{I}$. Then as $\left[X_{1}, X\right]=1$ and $J$ acts on $U \cap X$ for each $U \in \mathcal{W}$, (2) follows.

Assume the hypothesis of (3). As $Y_{1} \not \leq J$ and $\bar{Y}_{1}=\bar{X}$, it follows that $X \not \leq J$, so in particular $X \neq 1$, and hence $X$ is transitive on $\Omega_{X}$. By (2), $X \leq S_{I}$ so as $X$ is transitive on $\Omega_{X}, k=2$. By (2), $X \leq N_{G}(U \cap X)$ for each $U \in \mathcal{W}$, so in particular $J_{X}:=J \cap X \unlhd X$. Thus either $O^{2}(X) \leq J_{X}$ or $J_{X}=1$, or $\left|\Omega_{X}\right|=4$ and $J_{X}=O_{2}(X)$.

Suppose $O^{2}(X) \leq J$. Then as $X \not \leq J$, we have $X=T$, where $T=S_{\Omega-\Omega_{X}}$ and $J_{X}=O^{2}(X)$ is of index 2 in $X$. But then $|\bar{X}: J|=2$, contrary to 3.5.3. Thus $O^{2}(X) \not \leq J$. Thus $J_{X}=1$ or $O_{2}(X)$, so $J_{X} \leq O_{2}(X)$, and hence $X=O^{2}(X)$ by 3.5.2. Therefore $\hat{G}=A$, and then as $X$ is transitive on $\Omega_{X},\left|\Omega_{X}\right|>2$.

If $\left|\Omega_{X}\right| \neq 4$, then $X$ is the only nontrivial $X$-invariant subgroup of $T$, so as $X$ acts on $X \cap U$ for each $U \in \mathcal{W}$, it follows that $\mathcal{W}_{0}(<\bar{X})=\varnothing$, so $\bar{X} \in \mathcal{W}_{0}^{*}$. Thus (3) holds in this case. Finally suppose $\left|\Omega_{X}\right|=4$. Then

$$
W_{0}(<\bar{X})=\left\{O_{2}(X) J, X J\right\},
$$

so we conclude from 3.4 that (3) holds. This completes the proof of (3).

\subsection{Assume Hypothesis 10.24. Then $\bar{X} \notin W_{0}^{*}$.}

Proof. Assume $\bar{X} \in W_{0}^{*}$, let $\mathcal{C}$ be the connected component in $\Lambda^{\prime}$ of $\bar{X}$, and adopt the notation of 3.4. By 3.8 there is $V \in \mathcal{C}^{*}$ such that $V$ does not act on $X$, and hence does not act on $\Omega_{X}$. As $X \in W_{0}^{*}, X \not \leq J$, so $X \neq 1$ when $\left|\Omega_{X}\right|=2$, and therefore $X$ is transitive on $\Omega_{X}$. Indeed as $X \not \leq J,\left|\Omega_{X}\right|>2$ by 3.5.3.

Set $W:=\langle X, V\rangle$. As $\bar{X} \in \mathcal{W}_{0}^{*}$, we conclude from 3.4 that $W \in \mathcal{W}_{0}, \bar{X} \cap V=J$, and $\mathcal{W}_{0}(\leq W)=\{J, \bar{X}, V, W\}$. As $W$ does not act on $\Omega_{X}$, and as $H$ acts on $W$ and is transitive on $\Omega-\Omega_{X}, H X$ is transitive on $\Omega$. Then as $X \leq W$, it follows from 3.3 in [8] that $W$ is transitive on $\Omega$ and $W H$ preserves the partition $\Gamma:=\Omega_{X}^{W}$ of $\Omega$. As $X \leq W$ and $W$ is transitive on $\Omega$, it follows that $K:=\left\langle X^{W}\right\rangle \unlhd W$, and either $\hat{G}=A$ and $K=K_{+}(\Gamma)$, or $\hat{G}=S$ and $K=S_{\Gamma}$. In particular, $K$ acts on $\Omega_{X}$. Also $W=\langle X, V\rangle=K V$. Then by $2.7, \bar{X} \leq \bar{K} \in W_{0}$ with $\bar{K} \leq W$, and as $\bar{K}=K J$ acts on $\Omega_{X}$ but $V$ does not, it follows that $V \not \bar{K}$. Therefore as $\mathcal{W}_{0}(<W)=\{J, \bar{X}, V\}$, we conclude that $\bar{K}=\bar{X}$.

Next $K=K_{1} \times X$, where $K_{1}=K \cap X_{1}$. As $W=K V$ is transitive on $\Omega, V$ is transitive on $\Gamma$, so $K=\left\langle X^{V}\right\rangle=\left\langle K_{1}^{V}\right\rangle$. If $K_{1} \leq J$, then $K_{1} \leq V$, so

$$
K=\left\langle K_{1}^{V}\right\rangle \leq V \text {, }
$$

and hence $W=K V=V$, a contradiction. Hence $K_{1} \not \leq J$, so $\bar{K}_{1} \in \mathcal{W}_{0}^{\prime}$, and therefore $\bar{K}_{1}=\bar{K}=\bar{X}$ as $\bar{X} \in W_{0}^{*}$. Therefore by $10.27 .3, k=2, \hat{G}=A$, and $O_{2}(X)=J \cap X=J_{X}$. 
Next $V \cap K \leq V \cap \bar{X}=J$, so $V \cap K=J \cap K=: J_{K}$, and $J_{K}$ is $V$-invariant. As $\bar{K}=\bar{K}_{1}, K=K_{1} J_{K}$, so the projection $\pi_{X}: J_{K} \rightarrow X$ is a surjection. Then as $V$ is transitive on $\Gamma, \pi_{X} w: J_{K} \rightarrow X^{w}$ is a surjection for all $w \in W$. Therefore if $\left|\Omega_{X}\right|>4$, then by 1.4 in [7], $J_{K}$ is the product of full diagonal subgroups of $K$. Further $X \cap J=O_{2}(X)=1$, so $J_{K}$ is a complement to $X$ in $K$, and then we conclude that $K_{1} \cong J_{X} \cong X$ and $K_{1}=X_{1}$. But now $H_{*} \leq X_{1} \leq W$, a contradiction.

Therefore $\left|\Omega_{X}\right|=3$ or 4 . Then as $\bar{K}=\bar{X}$ and $X \cap J=O_{2}(X)$, it follows that $\left|K: J_{K}\right|=3$. Let $Q:=O_{2}(K)$ and $\tilde{K}:=K / Q$. Thus

$$
Q \leq J_{K} \unlhd K
$$

and $\left|\tilde{K}: \tilde{J}_{K}\right|=3$.

As $H_{*} \leq X_{1},\left|\Omega_{1}\right|>4$ by 10.3.2, so $|\Gamma|>2$. But $U=W \hat{G}_{\Gamma} \in W_{0}$ by 2.13 , as $\hat{G}_{\Gamma} / K$ is solvable and $W$ acts on $\hat{G}_{\Gamma}$. Thus $U \in \mathcal{C}$, so by $3.7, U$ acts on $J_{K}$. Now there exists $t$ in the kernel of the action of $\hat{G}$ on $\Gamma$ inverting exactly two members of $\tilde{X}^{W}=\{\tilde{X}(i): 1 \leq i \leq r\}$. Say $\left\langle\tilde{x}_{i}\right\rangle=\tilde{X}(i)$, and $t$ inverts $\tilde{x}_{j}$ for $j \in\{1,2\}$. Now as $\tilde{J}_{K}$ is a complement to $\tilde{X}(i)$ in $\tilde{K}$ for each $i, \tilde{X}(1) \tilde{X}(3) \cap \tilde{J}_{K}$ is a full diagonal subgroup of $\tilde{X}(1) \tilde{X}(3)$, and hence, replacing $x_{3}$ by $x_{3}^{-1}$ if necessary, is generated by $\tilde{x}=\tilde{x}_{1} \tilde{x}_{3}$. As $t \in U$ and $U$ acts on $J_{K}$, also $[\tilde{x}, t]=\tilde{x}_{1}$ is in $\tilde{J}_{K}$, contradicting $\tilde{X} \cap \tilde{J}_{K}=1$. This contradiction completes the proof.

10.29. Either

(1) $k=2$, or

(2) $k=3,\left|\Omega_{i}\right|=1$ for $i \in\{2,3\}$, and $X_{2,3}=1$, so $\hat{G}=A$.

Proof. By 10.5, $k \leq 3$, and in case of equality, Hypothesis 10.24 is satisfied by 10.25. Thus the lemma follows from 10.26 and 10.28 .

10.30. Assume Hypothesis 10.24 and suppose $\bar{X} \in \mathcal{W}_{0}^{\prime}$. Let $\mathcal{C}$ be the connected component of $\Lambda^{\prime}$ containing $\bar{X}, \varrho^{*}:=\bigodot \cap W_{0}^{*}$, and

$$
\varphi_{X}^{*}:=\left\{V \in \mathcal{C}^{*}: V \not N_{G}\left(\Omega_{X}\right)\right\} .
$$

Then $k=2$ and the following hold:

(1) $W_{0}(\leq \bar{X}) \cong \Delta(m)$ for some $m \geq 2$.

(2) The map $Z \mapsto \bar{Z}$ is an isomorphism of $\mathcal{V}_{X}(H)$ with $\mathcal{W}_{0}(\leq \bar{X})$.

(3) Let $\pi_{i}: T_{1} \times T_{2} \rightarrow T_{i}$ be the projection map. Then $X \pi_{2}^{-1} \cap H=H_{1} \times J_{X}$, where $H_{1}:=H \cap T_{1}$ and $J_{X}:=J \cap X$.

(4) $J_{X}$ is transitive on $\Omega_{X}$.

(5) $\bigodot_{X}^{*} \neq \varnothing$ and for each $V \in \mathcal{C}_{X}^{*}, V H$ is transitive and imprimitive on $\Omega$. 
Proof. By $10.28, \bar{X} \notin \mathcal{W}_{0}^{*}$, so (1) follows from the assumption that $\bar{X} \in \mathcal{W}_{0}^{\prime}$, and the fact that $\Lambda$ is a $D \Delta\left(m_{1}, \ldots, m_{t}\right)$-lattice. Part (2) follows from 3.7.

By (1), $X \neq 1$ and $\bar{X} \notin W_{0}^{*}$, so by $10.26, k=2$. Therefore $H$ is transitive on $\Omega_{1}$ and $\Omega_{X}=\Omega_{2}$.

Next as $H$ acts on $\Omega_{i}$ for $i=1,2, H \leq T_{1} \times T_{2}$. Let $Y_{i}:=H \pi_{i}$ be the projection of $H$ on $T_{i}$, and $H_{i}:=Y_{i} \cap X_{i}$. Now $H_{2} \in \mathcal{V}_{X}(H)$, so by (2), $\bar{H}_{2} \in \mathcal{W}_{0}$ and there exists a unique $B \in \mathcal{V}_{X}(H)$ with $X=\left\langle B, H_{2}\right\rangle$ and $B \cap H_{2}=J_{X}$. As $T_{1}$ centralizes $T_{2}, \mathcal{V}_{X}(H)=\mathcal{d}_{X}\left(Y_{2}\right) \cap \mathcal{O}_{X}\left(J_{X}\right)$. In particular each member of $\mathcal{V}_{X}(H)$ is $H_{2}$-invariant, so $B \unlhd\left\langle B, H_{2}\right\rangle=X$. Therefore we have $B=1$, or $O^{2}(X) \leq B$, or $\left|\Omega_{X}\right|=4$ and $B=O_{2}(X)$. In the first case $J_{X}=1$ and as $X=\left\langle B, H_{2}\right\rangle$, we get $H_{2}=X$. Then $\mathcal{V}_{X}(H)=d_{X}(X)$ is not isomorphic to $\Delta(m)$ for any $m \geq 2$, contrary to (1) and (2). In the second case, by 3.5.2, $B=X$, and then

$$
J_{X}=B \cap H_{2}=H_{2},
$$

so that (3) holds. In the third case $X=\mathrm{H}_{2} \mathrm{~B}=\mathrm{H}_{2} \mathrm{O}_{2}(X)$, so $\mathrm{H}_{2}$ is a complement to $O_{2}(X)$ in $X$, or $H_{2}=X$. The first case is impossible as $H$, and hence also $H_{2}$, is transitive on $\Omega_{X}$. The second is impossible as $\mathcal{V}_{X}(H) \cong \Delta(m)$ by (1) and (2). This completes the proof of (3).

As $H$ is transitive on $\Omega_{X}$, so is $Y_{2}$. Then as $\left|Y_{2}: H_{2}\right| \leq 2$ with $H_{2}=J_{X}$, either (4) holds or $\left|Y_{2}: J_{X}\right|=2$ and $Y_{2} \not J J$. In the latter case $\bar{Y}_{2} \in \mathcal{W}_{0}^{\prime}$ by 2.13 , contrary to 3.5.3. This establishes (4).

By 3.8, $\bigodot_{X}^{*} \neq \varnothing$. Let $V \in \bigodot_{X}^{*}$. As $H$ is transitive on $\Omega_{i}$ for $i=1,2$ and $V$ does not act on $\Omega_{2}, Y:=V H$ is transitive on $\Omega$. Suppose $Y$ is primitive. Then by (4) and 1.7.3, $Y$ is 2-transitive on $\Omega$ and either almost simple or affine. Thus $Y$ is affine by 2.14. Now $Y$ has a unique minimal normal subgroup $D$, and as $1 \neq V \unlhd Y$, $D \leq V$. As $D$ is transitive on $\Omega$ but $H$ is not, $D \not \leq J$, so $V=\bar{D}$ as $V \in \mathcal{W}_{0}^{*}$. Then

$$
Y=D J H=D H .
$$

But now 1.7.4 supplies a contradiction, establishing (5).

Before we leave the setup of Lemma 10.30, we obtain a contradiction to the hypothesis of the lemma. By (5), there is $\Gamma \in \mathcal{P}^{\prime}(Y)$. Pick $\Gamma$ minimal subject to this constraint. Thus $Y^{\Gamma}$ is primitive. By (4) and 1.1, $\Omega_{X}$ is a union of a set $\Gamma_{X}$ of blocks of $\Gamma$, and $J_{X}$ is transitive on $\Gamma_{X}$. As $H$ acts on $\Gamma_{X} \subset \Gamma$ and $Y$ is transitive on $\Omega, V^{\Gamma} \neq 1$, so $V^{\Gamma}$ is transitive. Similarly $V^{\Gamma} \neq J^{\Gamma}$, so $\bar{V} \neq \bar{V}_{\Gamma}$, and hence as $V \in \mathcal{W}_{0}^{*}, V_{\Gamma}=J_{\Gamma}$. Therefore $J_{\Gamma} \unlhd Y$.

Let $B:=V Y_{\Gamma}$ be the preimage of $V^{\Gamma}$ in $Y$, and assume $H_{*} \not \subset B$. Then $B \in \mathcal{C}$ by 2.12. Then $B=B \cap Y=B \cap V H=V(B \cap H)=V J=V$; that is $Y_{\Gamma} \leq V$.

Let $\gamma \in \Gamma_{X}$ and suppose $\{\gamma\} \neq \Gamma_{X}$. Then as $J_{X}^{\Gamma}$ is transitive on $\Gamma_{X}$ and $Y^{\Gamma}$ is primitive, applying 1.7.3 to $Y^{\Gamma}$ and arguing as above in the proof of part (5), 
using the fact that $Y_{\Gamma} \leq V$, we conclude that $Y^{\Gamma}$ is 2-transitive and affine with $F^{*}\left(Y^{\Gamma}\right) \leq V^{\Gamma}$. Then continuing as in the proof of (5), we obtain a contradiction.

Therefore $\Gamma_{X}=\{\gamma\}$, so $\Omega_{X}=\gamma \in \Gamma$. As $H_{*} \leq Y$ and $H_{*} \not Z \geq Y_{\Gamma}, H_{*}$ is not contained in the preimage $E$ of $V^{\Gamma}$ in $N_{\hat{G}}(\Gamma)$. Therefore by $2.12, \bar{E} \in \mathcal{C}$. For $\alpha \in \Gamma$, set $E(\alpha):=E_{\Omega-\alpha}$. Then $X=E(\gamma)$, so as $V$ is transitive on $\Gamma$,

$$
X^{V}=\{E(\alpha): \alpha \in \Gamma\}=\mathcal{X} .
$$

Set $F:=\langle X\rangle$. Applying 3.7 to $F, F H$ in the role of $Y, X$, we conclude that $V$ acts on $\bar{X} \cap F=X(J \cap F)$ and $J_{F}:=J \cap F$. As $V$ acts on $X J_{F}, X J_{F}=F$. As $V$ and $H$ act on $J_{F}$, so does $Y$. For $\alpha \in \Gamma$, let $\pi_{\alpha}: F \rightarrow E(\alpha)$ be the projection. As $X J_{F}=F$, for $\alpha \in \Gamma-\{\gamma\}, J_{F} \pi_{\alpha}=E(\alpha)$, so as $J_{F} \unlhd Y$ and $V$ is transitive on $\Gamma$, also $X=J_{F} \pi_{\gamma}$. Therefore as $J_{X} \unlhd J_{F}$, we also have $J_{X} \unlhd X$. Therefore either $O^{2}(X) \leq J$ or $m_{X}:=\left|\Omega_{X}\right|=4$ and $J_{X}=O_{2}(X)$. By 3.5.2,

$$
X=O^{2}(X) J_{X},
$$

so as $X \not \leq J$, it follows that $O^{2}(X) \not \leq J$. Thus $m_{X}=4$ with $O_{2}(X)=J_{X}<X$ and $X=O^{2}(X) J_{X}$. But now (1) and (2) supply a contradiction.

We have shown that $H_{*} \leq B$. Suppose next that $\Gamma_{X} \neq\{\gamma\}$. Then

$$
Z:=X_{\Gamma_{X}} \in d_{X}(H),
$$

so by 3.4, $W=\langle Z, V\rangle \in \mathcal{C}$. As $W H$ acts on $\Gamma$, it follows from 3.2.2 in [8], that $K_{+}(\Gamma) \leq W$. Let $K$ be the kernel of the action of $N_{\hat{G}}(\Gamma)$ on $\Gamma$. As $K / K_{+}(\Gamma)$ is solvable, $K W \in \mathcal{W}_{0}$ by 2.13 , so $K \leq W$. Then as $V \leq W$, the preimage $B_{0}$ of $V^{\Gamma}$ in $W H$ is contained in $W$. As $H_{*} \leq B \leq B_{0}$, this is a contradiction. Therefore $\Gamma_{X}=\{\gamma\}$, where $\Omega_{X}=\gamma \in \Gamma$.

Let $\mathcal{Z}:=\left\{Z \in \mathcal{V}_{X}(H): \bar{Z} \in \mathcal{W}_{0}^{*}\right\}$, and for $Z \in \mathcal{Z}$, set $W(Z):=\langle Z, V\rangle$. By 3.4, $W(Z) \in \mathcal{C}$, and by construction, $Z \leq W(Z)_{\Gamma}$, so $W(Z)=W(Z)_{\Gamma} V$. Then as $V \in W_{0}$ and $V_{\Gamma}=J_{\Gamma}$, it follows that $\overline{W(Z)_{\Gamma}}=\bar{Z}$. Thus $W(Z)_{\Gamma}=Z J_{\Gamma}$ is

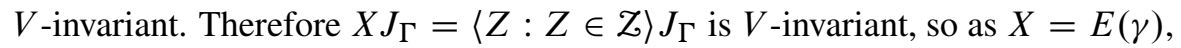

$$
F=\prod_{\alpha \in \Gamma} E(\alpha)=\left\langle X^{V}\right\rangle=X J_{\Gamma}
$$

Let $P:=Y F$. Now either $\hat{G}=A$ and $F=K_{+}(\Gamma)$, or $\hat{G}=S$ and $F=S_{\Gamma}$. In either case, $P_{\Gamma} / F$ is solvable, so $P_{\Gamma} V \in W_{0}$ by 2.13. This is a contradiction as $H_{*} \leq B \leq P_{\Gamma} V$.

This contradiction shows:

10.31. Assume Hypothesis 10.24. Then $X \leq J$. 
10.32. Assume Hypothesis 10.24 and let

$$
\mathcal{W}_{1}=\left\{W \in \mathcal{W}_{0}: W \not \leq N_{G}\left(\Omega_{1}\right)\right\} \quad \text { and } \quad \mathcal{W}_{1}^{*}=\mathcal{W}_{1} \cap \mathcal{W}_{0}^{*} .
$$

Then:

(1) $W_{1}^{*} \neq \varnothing$.

(2) For each $W \in \mathcal{W}_{1}$, there exists $U \in W_{1}^{*}$ with $U \leq W$.

Proof. Part (1) follows from 3.3. Let $W \in \mathcal{W}_{1}$. As $J$ acts on $\Omega, W \neq J$. Then (2) follows from 3.4.

10.33. Assume Hypothesis 10.24 and $X \neq 1$. Let $W \in \mathcal{W}_{1}, U \in W_{1}^{*}$ with $U \leq W$. Set $Y=W H$. Then:

(1) $X$ is transitive on $\Omega_{X}$.

(2) $W$ is transitive on $\Omega$.

(3) $\Gamma=\Gamma_{W}:=\Omega_{X}^{W}$ is a $X$-invariant partition of $\Omega$ and $\Gamma_{W}=\Gamma_{U}$.

(4) $K=K_{+}(\Gamma) \leq J$.

(5) $\hat{G}_{\Gamma} W \in W_{0}$.

(6) $Y^{\Gamma}$ is 2-transitive and affine on $\Gamma, Y_{\Gamma} \leq W$, and $F^{*}\left(Y^{\Gamma}\right) \leq W^{\Gamma}$.

(7) $F^{*}\left((W H)^{\Gamma}\right)=F^{*}\left((U H)^{\Gamma}\right)$.

(8) Let $D_{W}$ be the preimage in $N_{\hat{G}}(\Gamma)$ of $F^{*}\left(Y^{\Gamma}\right)$. Then we have $D_{W}=D_{U}$ and $\bar{D}_{W} \in W_{0}$.

Proof. As $X \neq 1$ and $X$ contains the group of even permutations in $T_{2}$, (1) holds. Then as $H$ is transitive on $\Omega_{1}$ and $W$ does not act on $\Omega_{1}, Y:=W H$ is transitive on $\Omega$. Now by 3.2 in [8], $\mathcal{P}^{\prime}(Y)$ has a greatest member $\Gamma=\Gamma_{W}, \Omega_{X} \subseteq \alpha \in \Gamma$, and

$$
K=K_{+}(\Gamma) \leq W .
$$

As $H$ is transitive on $\Omega_{1}$, it follows that $\alpha=\Omega_{X}, 1 \neq W^{\Gamma}$, and $Y^{\Gamma}$ is 2-transitive. Therefore $W^{\Gamma}$ contains the unique minimal normal subgroup $D^{\Gamma}$ of $Y^{\Gamma}$, and $D^{\Gamma}$ is transitive. As $X \leq W$ and $X$ is transitive on $\alpha$, (2) follows. Then

$$
\Gamma=\alpha^{W}=\Omega_{X}^{W}
$$

By symmetry between $W$ and $U, U$ is transitive on $\Omega$ and $\Gamma_{U}=\Omega_{X}^{U}$. As $U$ is transitive on $\Omega$, it is also transitive on $\Gamma$, so $\Gamma=\Omega_{X}^{U}$, and hence $\Gamma=\Gamma_{U}$, completing the proof of (3).

Again by symmetry between $U$ and $W, K \leq U$. As $J$ is not transitive on $\Gamma$ but $U$ is transitive, $J^{\Gamma} \neq U^{\Gamma}$, so $U_{\Gamma} \leq J$. Thus $\bar{K} \leq U_{\Gamma} \leq J$, establishing (4). 
As $\hat{G}_{\Gamma} / K$ and $Y_{\Gamma} / K$ are 2-groups, $W \hat{G}_{\Gamma}$ and $W Y_{\Gamma}$ are in $\mathcal{W}_{0}$ by 2.13, so (5) holds. Then $W Y_{\Gamma}=W H \cap W Y_{\Gamma}=W\left(H \cap W Y_{\Gamma}\right)=W J=W$, so $Y_{\Gamma} \leq W$.

If $Y^{\Gamma}$ is almost simple, then

$$
H_{*}^{\Gamma} \leq D^{\Gamma} \leq W^{\Gamma},
$$

so as $Y_{\Gamma} \leq W, H_{*} \leq W$, a contradiction. Thus (6) holds as $Y^{\Gamma}$ is 2-transitive.

Part (7) follows from (3), (6), and symmetry between $U$ and $W$. Then (8) follows from (5) and (7).

10.34. Assume Hypothesis 10.24. Then $X=1$.

Proof. Assume otherwise. By 10.32.1, there is $U \in W_{1}^{*}$. Adopt the notation of 10.33 , and let $\Gamma:=\Gamma_{U}$ and $D:=D_{U}$. Let $\ell$ be the connected component of $\Lambda^{\prime}$ containing $U$, and $\mathcal{C}^{*}:=\ell \cap W_{0}^{*}$. Then for each $U \leq W \in W_{0}, W \in \mathcal{C} \cap W_{1}$, and by $10.33, \Gamma=\Gamma_{W}$ and $D=D_{W}$. But for each $V \in \mathcal{C}^{*}, U \leq\langle U, V\rangle \in \mathcal{W}_{0}$, so $V \leq W \leq N_{G}(D)$. Thus $\ell \subseteq N_{G}(D)$, so 3.8 supplies a contradiction.

10.35. Assume Hypothesis 10.24. Then one of the following holds:

(1) $k \leq 3,\left|\Omega_{X}\right|=2$, and $\hat{G}=A$.

(2) $k=2$ and $\left|\Omega_{X}\right|=1$.

Proof. By $10.34, X=1$, so $\left|\Omega_{X}\right| \leq 2$, and in case of equality, $\hat{G}=A$. As $k \leq 3$ by 10.5 , the lemma follows.

10.36. Assume Hypothesis 10.24. Let $W \in \mathcal{W}_{1}$, and let $\Omega_{W}$ be the orbit of

$$
Y:=W H
$$

on $\Omega$ containing $\Omega_{1}$. Then one of the following holds:

(1) $\left|\Omega_{W}\right|=\left|\Omega_{1}\right|+1$, and $Y$ is 2-transitive and affine on $\Omega_{W}$. Thus

$$
F^{*}(Y)=: D_{W}
$$

is regular on $\Omega_{W}$, and $H$ is transitive on $D_{W}^{\#}$ via conjugation. Further

$$
\bar{D}_{W} \in W_{0}^{*} .
$$

(2) $\left|\Omega_{X}\right|=2, \Omega_{W}=\Omega, \Gamma=\Gamma_{W}=\Omega_{X}^{W}$ is a $Y$-invariant partition of $\Omega, \hat{G}=A$, $\hat{G}_{\Gamma} J \in \mathcal{W}_{0}-\mathcal{W}_{1}, \hat{G}_{\Gamma} W \in \mathcal{W}_{0}, Y_{\Gamma} \leq W$, and $Y^{\Gamma}$ is 2-transitive and affine. Let $D_{W}$ be the preimage in $W$ of $F^{*}\left(Y^{\Gamma}\right)$. Then $\bar{D}_{W} \in \mathcal{W}_{0}^{\prime}$ and $H^{\Gamma}$ is transitive on $D^{\Gamma \#}$. 
(3) $k=2, \Omega_{X}=\left\{\alpha_{1}, \alpha_{2}\right\}$ is of order $2, \Omega_{W}=\Omega, H_{\alpha_{1}}$ has two orbits $\theta_{i}, i=1,2$, on $\Omega_{1}$, and setting

$$
\Xi_{i}:=\theta_{i} \cup\left\{\alpha_{i}\right\} \text { and } M:=N_{W H}\left(\Xi_{1}\right) \text {, }
$$

$\Xi_{W}:=\left\{\Xi_{1}, \Xi_{2}\right\} \in \mathcal{P}^{\prime}(Y), \hat{G}=A$, and $M^{\Xi_{i}}$ is 2-transitive and affine. Let $D_{i}$ be the projection of $F^{*}\left(M^{\Xi_{i}}\right)$ on $G_{\Omega-\Xi_{i}}$ and $D_{W}:=D_{1} D_{2}$. Then we have $\bar{D}_{W} \in W_{0}^{\prime}$ and $F^{*}(Y)$ is $D_{W}$ or a full diagonal subgroup of $D_{W}$.

Proof. As $W \in W_{1}, \Omega_{1}$ is a proper subset of $\Sigma:=\Omega_{W}$. But by $10.21,\left|\Omega_{X}\right| \leq 2$, so either $|\Sigma|=\left|\Omega_{1}\right|+1$, or $\left|\Omega_{X}\right|=2$ and $\Sigma=\Omega$. Assume the first case holds. Then as $H$ is transitive on $\Omega_{1}, Y$ is 2-transitive on $\Sigma$, so by 2.15, $Y$ is affine on $\Sigma$, and setting $D_{W}:=F^{*}(Y), D_{W} \leq W$ with $\bar{D}_{W} \in \mathcal{W}_{0}$. As $H$ is transitive on $\Omega_{1}$, $H$ is transitive on $D_{W}^{\#}$ by conjugation. In particular $\bar{D}_{W} \in \mathcal{W}_{1}$ and $H$ is maximal in $\bar{D} H=D H$, so we have $\bar{D}_{W} \in \mathcal{W}_{0}^{*}$. Thus (1) holds in this case.

So assume the second case holds. Suppose $\Gamma=\Omega_{X}^{W}$ is a partition of $\Omega$, and let $K=\hat{G}_{\Gamma}$. As $K$ is an $H$-invariant 2-group, $\bar{K}$ and $K W$ are in $\mathcal{W}_{0}$ by 2.13 , and by construction $\bar{K}=K J$ acts on $\Omega_{1}$, so $\bar{K} \notin W_{1}$. As $X=1$ by $10.34, \hat{G}=A$. As $Y_{\Gamma} \leq K$ and $K W \in \mathcal{W}_{0}, W Y_{\Gamma} \in \mathcal{W}_{0}$ by 2.7 , so

$$
W Y_{\Gamma}=H W \cap W Y_{\Gamma}=W\left(H \cap W Y_{\Gamma}\right)=W J=W .
$$

That is $Y_{\Gamma} \leq W$.

As $\bar{K} \notin \mathcal{W}_{1}, W^{\Gamma} \neq 1$. As $Y$ is transitive on $\Omega$ and $H$ is transitive on $\Gamma-\left\{\Omega_{X}\right\}$, $Y^{\Gamma}$ is 2-transitive. Then as $W^{\Gamma} \neq 1$ and $Y_{\Gamma} \leq W, Y^{\Gamma}$ is affine by 2.15. Define $D=D_{W}$ as in (2). Then $\bar{D} \in \mathcal{W}_{0}$ by 2.15 . As $D$ does not act on $\Omega_{1}, \bar{D} \in \mathcal{W}_{0}^{\prime}$. As $H$ is transitive on $\Omega_{1}, H^{\Gamma}$ is transitive on $D^{\Gamma \#}$. Thus (2) holds in this case.

Thus we may assume $\Gamma$ is not a partition of $\Omega$. It follows from 1.6 that case (i) or (ii) of 1.6.1 holds. Suppose that case (i) holds. Then $Y$ is 5/2-transitive on $\Omega$. By $2.14, Y$ is affine, so by 1.6.2, $D:=F^{*}(Y) \cong E_{2^{e}}$ is regular on $\Omega$, and for $\omega \in \Omega_{X}, Y_{\omega}$ is isomorphic to $L_{e}(2)$, or possibly $A_{7}$ if $e=4$. Then as $Y / W$ is almost simple, we conclude $W=D$ and $Y_{\omega}=H=H_{*}$. But now $H$ is transitive on $\Omega-\{\omega\}$, whereas $H$ acts on $\Omega_{X}$ of order 2 , a contradiction.

Thus we may assume case (ii) holds. Then, together with 1.6.3, this implies that (3) holds, except for the condition that $\bar{D}_{W} \in \mathcal{W}_{0}$, which follows from 2.13 .

10.37. $k=2$ and $I_{*}^{\prime}=\varnothing$.

Proof. Assume otherwise. If $k=2$, we may assume $I_{*}^{\prime}$ is nonempty, so Hypothesis 10.24 is satisfied. On the other hand if $k \neq 2$, then by $10.29, k=3$. Then from the discussion in 10.25, Hypothesis 10.24 is satisfied.

So in any event, Hypothesis 10.24 is satisfied. By 10.32 .1 we may choose

$$
W \in \mathcal{W}_{1} \text {. }
$$


Define $\Omega_{W}$ and $Y:=W H$ as in 10.36 . By 10.36 one of the three conclusions of that lemma are satisfied. Let $\mathcal{C}$ be the connected component of $\Lambda^{\prime}$ containing $W$ and $\ell^{*}:=\ell \cap W_{0}^{*}$.

Suppose first that conclusion (1) of 10.36 is satisfied, and set $D:=D_{W}$. Then $\bar{D} \in \mathcal{W}_{0}^{*}$ by 10.36 . Let $D \leq Z \in \mathcal{W}_{0}$. As $D$ does not act on $\Omega_{1}, Z \in \mathcal{W}_{1}$. We claim $\Omega_{W}=\Omega_{Z}$, so $Z$ satisfies conclusion (1) of 10.36. If not $\Omega_{Z}=: \Omega$ is of order $\left|\Omega_{1}\right|+2$, so as $D H$ is 2-transitive on $\Omega_{W}$ of order $\left|\Omega_{1}\right|+1, Z H$ is 3-transitive on $\Omega$, impossible as $Z H$ is imprimitive on $\Omega$ in cases (2) and (3) of 10.36 . Thus the claim is established.

By the claim, $\Omega_{Z}=\Omega_{\bar{D}}=\Omega_{W}$, and then as $D H \leq Z H$,

$$
D=F^{*}(D H)=F^{*}(Z H)=: D_{Z} \text {. }
$$

Let $V \in \mathcal{C}^{*}$ and $Z(V)=\langle D, V\rangle$. As $\bar{D} \in \mathcal{W}_{0}^{*}$, it follows that $Z(V) \in \mathcal{C}$ by 3.4 , so $D=D_{Z(V)}$. Thus $V \leq N_{G}(D)$ for each $V \in \mathcal{C}^{*}$, so $\ell \leq N_{G}(D)$, contrary to 3.8. Thus no member of $\mathcal{W}_{1}$ satisfies conclusion (1) of 10.36 .

Next suppose $W$ satisfies conclusion (2) of 10.36. Adopt the notation in (2) and let $K:=\hat{G}_{\Gamma}$ and $D:=D_{W}$. Let $\tau \in S$ be the involution with cycles $\left(\omega, \omega^{\prime}\right)$, for $\left\{\omega, \omega^{\prime}\right\} \in \Gamma$, and set $T=\langle\tau\rangle$. Let $\tau^{\prime}=\tau \cdot\left(\omega_{1}, \omega_{2}\right)$, where $\Omega_{X}=\left\{\omega_{1}, \omega_{2}\right\}$.

Suppose first that $B \in \mathcal{d}_{K}(H)$ with $B \not T$. Claim there in no $\Xi \in \mathcal{P}(B H)$ with $\Xi=\left\{\Xi_{1}, \Xi_{2}\right\}$ of order 2 . For if $\gamma \in \Gamma^{\prime}=\Gamma-\left\{\Omega_{X}\right\}$ with $\gamma \subseteq \Xi_{1}$, then as $H$ is transitive on $\Gamma^{\prime}$, it follows that $\Omega_{1} \subseteq \Xi_{1}$, contradicting

$$
\left|\Xi_{1}\right|=n / 2<n-2=\left|\Omega_{1}\right| \text {. }
$$

We conclude for each $\gamma \in \Gamma^{\prime},\left|\Xi_{i} \cap \gamma\right|=1$ for $i=1,2$, and then also

$$
\left|\Xi_{i} \cap \Omega_{X}\right|=1 \text {. }
$$

Let $b \in B-T$. Then there are $\alpha, \beta \in \Gamma$ with $\alpha \subseteq M(b)$ and $\beta \subseteq \operatorname{Fix}(b)$. As $\alpha \subseteq M(b)$ and $\left|\Xi_{1} \cap \alpha\right|=1, \Xi_{1} b=\Xi_{2}$. This is impossible as $b$ fixes the member of $\Xi_{1} \cap \beta$, establishing the claim.

Suppose next that for each $Z \in W_{0}$ containing $W, Z H$ satisfies conclusion (2) of 10.36; for example if $W_{\Gamma} \not \leq T$ this holds by the previous paragraph. Let

$$
W \leq Z \in \mathcal{W}_{0} .
$$

As $W$ does not act on $\Omega_{1}$, it follows that $Z \in \mathcal{W}_{1}$. By hypothesis, $Z$ satisfies conclusion (2) of 10.36. Then $\Gamma_{Z}=\Omega_{X}^{Z}=\Omega_{X}^{W}=\Gamma$, so $K=\hat{G}_{\Gamma_{Z}}$ and then by Proposition 4 in [4], $D=D_{Z}$. Thus $Z \leq N_{G}(D)$, and we obtain a contradiction as in the previous paragraph unless $W \in W_{0}$.

By 10.36 , we have $\bar{D} \in \mathcal{W}_{0}^{\prime}$. By 3.7 there is $D_{0} \leq \mathcal{V}_{D}(H)$ such that $\bar{D}_{0} \in \mathcal{W}_{0}^{*}$ and $D_{0}^{\Gamma} \neq 1$. As $H$ is irreducible on $D^{\Gamma}$, it follows that $D_{0}^{\Gamma}=D^{\Gamma}$, so $\bar{D}_{0}$ satisfies conclusion (2) of 10.36 with $D_{D_{0}}=D$. Replacing $W$ by $\bar{D}_{0}$, we may as- 
sume $W=\bar{D}_{0} \in \mathcal{W}_{0}^{*}$. Then $W \notin \mathcal{W}_{0}^{!}$, so $W_{\Gamma} \leq T$ by the previous paragraph. In particular $J_{\Gamma} \leq T$.

By $10.36, W K \in \mathcal{W}_{0}$, so by 3.7 there is $K_{0} \in \mathcal{V}_{K}(H)$ with $K_{0} \not \leq T, \bar{K}_{0} \in \mathcal{W}_{0}^{*}$, and $Z:=\left\langle W, K_{0}\right\rangle \in W_{0}$. By the claim, $Z$ acts on no partition of order 2, so we

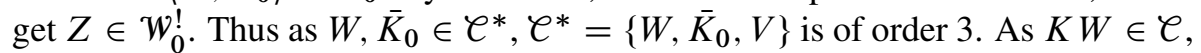
it follows that $\bar{K}=\bar{K}_{0}$, so $K=K_{0} J_{\Gamma} \leq K_{0} T$. In particular $H$ is irreducible on $K T / T$, so it follows that $n / 2$ is even (or else $\tau^{\prime} \in C_{K}(H)$ ) and hence $\tau \in A$, so that $T \leq K$ is $H$-invariant. Then $T=J_{\Gamma}$ by 3.5.3.

By 3.8, $V$ does not act on $\Gamma$. Next $U:=\langle V, W\rangle \in \mathcal{C}$, and as $V$ does not act on $\Gamma$, by an earlier reduction, $U H$ does not satisfy conclusion (2) of 10.36, so $U H$ satisfies conclusion (3) of 10.36. Thus $M:=N_{U H}\left(\Xi_{1}\right)$ is of index 2 in $U H$, and $D=D_{M} \times T$, where $D_{M}:=D \cap M \cong D^{\Gamma}$. Then $D_{M} \cong D_{M}^{\Xi_{1}}=F^{*}\left(M^{\Xi_{1}}\right)$. As in 10.36 .3 , let $D_{i}$ be the projection of $D_{M}$ on $G_{\Omega-\Xi_{i}}$ and $D_{U}:=D_{1} D_{2}$. Now $\bar{D}_{U} \in W_{0}$ by 10.36 and $Y=C_{U H D_{U}}(\tau)$, so $W=\bar{D}_{M}<\bar{D}_{W}$ and $J \cap D_{U}=1$. Thus $U=\bar{D}_{U}$ so as $V \in W_{0}^{*}, V=\bar{D}_{V}$, where $D_{V}:=V \cap D_{U}$. Thus by 3.4 and 3.7, $U H=D_{U} H$ and $D_{U}=D_{M} \times D_{V}$. Finally $\tau \in J$, so $\tau$ acts on $D_{V}$. However as $\tau$ centralizes $H$ and acts on $V$, and as $H$ is irreducible on $D_{V}$,

$$
D_{V} \leq C_{D_{U}}(\tau)=D_{M},
$$

a contradiction.

Therefore conclusion (3) of 10.36 is satisfied for each $W \in \mathcal{W}_{1}$. Set

$$
Y_{W}:=W H \text {. }
$$

Then we have $\Xi_{W}=\left\{\Xi_{1}, \Xi_{2}\right\}$ with $\Xi_{i}=\Xi_{W, i}$ the orbits of $F^{*}\left(M_{W}\right)$ on $\Omega$, where $M_{W}:=N_{Y_{W}}\left(\Xi_{1}\right)$, and $M_{W}^{\Xi_{i}}$ is affine.

Pick $W \in W_{0}^{*}$ and let $W \leq Z \in \mathcal{W}_{0}$. As $W \in W_{0}^{*}$, it follows that $W=\bar{E}$, where $E:=W \cap D_{W}$, and $D_{W}=D_{1} \times D_{2}$ is defined in 10.36.3. As $E$ is contained in each subgroup of $Y_{W}$ of index 2, $E \leq M_{Z}$. Thus $E$ acts on $\Xi_{Z, i}$ of order $n / 2$, so as the orbits $\Xi_{W, i}$ of $E$ are of order $n / 2$, it follows that $\Xi_{Z}=\Xi_{W}=\Xi$. By Proposition 4 in [4], $E^{\Xi_{i}}=F^{*}\left(M_{Z}^{\Xi_{i}}\right)$, so $D=D_{W}=D_{Z}$.

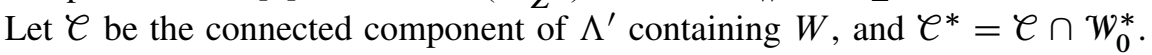
For each $V \in \mathcal{C}^{*}, W \leq Z=\langle V, W\rangle \in \mathcal{C}$, so $V \leq Z \leq N_{G}(D)$ by the previous paragraph. This contradicts 3.8 , and completes the proof.

10.38. Set $H_{i}:=H_{*} \pi_{i}$ and $J_{i}:=J_{*} \pi_{i}$. Then:

(1) $k=2, I_{*}=\{1,2\}, m_{i}=\left|\Omega_{i}\right| \geq 5$, and $H_{i} / J_{i} \cong L$.

(2) $\bar{H}_{i}$ and $\bar{X}_{i}$ are in $W_{0}^{\prime}$.

(3) $H_{i}=F^{*}\left(X_{i}\right)$.

(4) $X_{i} \in W_{0}^{*}$. 
Proof. By 10.37, $k=2$ and $I_{*}=\{1,2\}$, so (1) follows from part (2) of 10.3.

As $I_{*}=\{1,2\}, i \in \mathcal{S}$, so $\bar{X}_{i} \in \mathcal{W}_{0}$ by 10.3.3. As $J_{i} \neq H_{i}$, we have $H_{i} \not \leq J$, so $\bar{H}_{i} \neq J$, and hence (2) holds.

Let $Y_{i}=F^{*}\left(X_{i}\right)$. As $m_{i} \geq 5, Y_{i} \cong A_{m_{i}}$ is nonabelian simple and of index at most 2 in $X_{i}$. By (2) and 2.7, $\bar{Y}_{i} \in \mathcal{W}_{0}$, and then by 3.5.2, $\bar{X}_{i}=\bar{Y}_{i}$. If $\bar{X}_{i} \in \mathcal{W}_{0}^{*}$, then (4) holds and (3) follows from (2), so we may assume otherwise. Then by 3.7 there exists $Z \in \mathcal{V}_{X_{i}}(H)$ with $\bar{Z} \in \mathcal{W}_{0}^{\prime}, X_{i}=\left\langle H_{i}, Z\right\rangle$, and $H_{i} \cap Z \leq J$. As $Z$ is $H_{*}$-invariant, $H_{i}$ acts on $Z$, so $Z \unlhd\left\langle H_{i}, Z\right\rangle=X_{i}$, and hence $Y_{i} \leq Z$, contradicting $Z \cap H_{i} \leq J$. This completes the proof of (3) and (4).

Theorem 10.39. Assume Hypothesis 5.1. Then $H$ is transitive on $\Omega$.

Proof. Assume otherwise. Then Hypothesis 10.1 is satisfied, so we can appeal to the results in this section. Adopt the notation of 10.38, and choose notation so that $m_{1} \geq m_{2}$. Let $\mathcal{C}$ be the connected component of $\Lambda^{\prime}$ containing $\bar{X}_{1}$ and

$$
e^{*}:=e \cap w_{0}^{*} .
$$

By 3.8, there exists $V \in \mathcal{C}^{*}$ such that $V \not N_{G}\left(\Omega_{1}\right)$. By 10.38.4, $\bar{X}_{1} \in W_{0}^{*}$, so by 3.4 ,

$$
W:=\left\langle X_{1}, V\right\rangle \in W_{0} .
$$

Set $Y:=W H$. As $H$ is transitive on $\Omega_{i}$ and $Y \not \leq N_{G}\left(\Omega_{1}\right)$, it follows that $Y$ is transitive on $\Omega$. Then as $X_{1} \leq W$ and $m_{1} \geq 5$, it follows from 3.2 in [8] that there is $\Gamma \in \mathcal{P}^{\prime}(Y)$ with $\Omega_{1} \subseteq \gamma \in \Gamma$ and $K_{+}(\Gamma) \leq W$. As $m_{1} \geq m_{2}$, it follows that $m_{1}=n / 2$ and $\Gamma=\left\{\Omega_{1}, \Omega_{2}\right\}$. But now

$$
O^{2}\left(X_{2}\right) \leq K_{+}(\Gamma) \leq W,
$$

so $H_{*} \leq X_{1} O^{2}\left(X_{2}\right) \leq W$, contradicting $W \in \mathcal{W}_{0}$. This contradiction completes the proof of the theorem.

\section{Bibliography}

[1] M. Aschbacher, Finite Group Theory, Cambridge University Press, Cambridge, 1986.

[2] M. Aschbacher, Projective planes, loops, and groups, J. Algebra 300 (2006), 396432.

[3] M. Aschbacher, On intervals in subgroup lattices of finite groups, J. Amer. Math. Soc. 21 (2008), 809-830.

[4] M. Aschbacher, Overgroups of primitive groups, J. Aust. Math. Soc. 87 (2009), 3782. 
[5] M. Aschbacher, Overgroups of primitive groups, II, J. Algebra 322 (2009), 15861626.

[6] M. Aschbacher, Signalizer lattices in finite groups, Michigan J. Math. 58 (2009), 79-103.

[7] M. Aschbacher and L. Scott, Maximal subgroups of finite groups, J. Algebra 92 (1985), 44-80.

[8] M. Aschbacher and J. Shareshian, Restrictions on the structure of subgroup lattices of alternating and symmetric groups, J. Algebra 322 (2009), 2449-2463.

[9] M. Aschbacher and S. Smith, The Classification of Quasithin Groups, Mathematical Surveys and Monographs 11, American Mathematical Society, Providence, RI, 2004.

[10] R. Baddeley and A. Lucchini, On Representing finite lattices as intervals in subgroup lattices of finite groups, J. Algebra 196 (1997), 1-100.

[11] D. Gorenstein, R. Lyons and R. Solomon, The Classification of the Finite Simple Groups. Number 3, Mathematical Surveys and Monographs 40, American Mathematical Society, Providence, RI, 1999.

[12] M. Liebeck, C. Praeger and J. Saxl, A classification of the maximal subgroups of the finite alternating and symmetric groups, J. Algebra 111 (1987), 365-383.

[13] P. Palfy and P. Pudlak, Congruence lattices of finite algebras and intervals in subgroup lattices of finite groups, Algebra Universalis 11 (1980), 22-27.

[14] P. Perepelitsky, Intervals in subgroup lattices of the alternating and symmetric groups of prime degree, J. Group Theory 12 (2009), 119-137.

[15] J. Shareshian, Topology of order complexes of intervals in subgroup lattices, $J$. Algebra 268 (2003), 677-686.

Received September 18, 2010; revised November 28, 2011.

\section{Author information}

Michael Aschbacher, California Institute of Technology,

Pasadena, California 91125, USA. 